\title{
External Ellipsoidal Approximations for Set Evolution Equations
}

\author{
Steven Duda ${ }^{1} \cdot$ Edeltraud Gehrig ${ }^{1} \cdot$ Thomas Lorenz $^{1}$ (D)
}

Received: 30 May 2021 / Accepted: 2 December 2021 / Published online: 12 February 2022

(c) The Author(s) 2022

\begin{abstract}
In many applications, uncertainty and imprecision in control systems require the focus on reachable sets instead of single state vectors. Then, closed-loop controls also refer to these attainable sets leading to a class of set evolution problems. We suggest sufficient conditions for its well-posedness and for approximating their solutions by intersections of finitely many time-dependent ellipsoids characterized by solutions to a system of ordinary differential equations.
\end{abstract}

Keywords Set differential equations - Morphological equations · Reachable sets · Integral funnel $\cdot$ Ellipsoidal approximations $\cdot$ Guaranteed state estimation

Mathematics Subject Classification 34A60 - 34G25 · 49J53 · 93B03 · 93C41

\section{Introduction}

The focus is the external ellipsoidal approximation of all attainable states in a control system with uncertainties as they occur in many technical applications. This arouses our interest in reachable sets (instead of single state vectors) and we face the challenge that these sets have a feedback influence on the underlying control system and thus on their own evolution. The results here lay the foundations for future descent methods in optimization problems.

Communicated by Felix L. Chernousko.

Thomas Lorenz

thomas.lorenz@hs-rm.de

Steven Duda

steven.duda@student.hs-rm.de

Edeltraud Gehrig

edeltraud.gehrig@hs-rm.de

1 Applied Mathematics, RheinMain University of Applied Sciences, 65022 Wiesbaden, Germany 
As a first motivation, we sketch the optimization problem of output feedback control (OFC) in uncertain systems introduced by KURZHANSKI and VARAIYA [35] (see also [36, Ch. 10]). In its linear (or linearized) form without available measurements, the state $x(t) \in \mathbb{R}^{n}$ is determined by $x^{\prime}=A(t) x+B(t) \eta+C(t) v$ with a control $v(t) \in V$ and bounded "unknown noise" $\eta(t)$. Furthermore, the initial set $K_{0} \subset \mathbb{R}^{n}$ and the setvalued map $U(\cdot)$ of constraints on $\eta(t)$ are given. For each control $v \in L^{1}([0, T], V)$, the guaranteed state estimation $K_{v}(t) \subset \mathbb{R}^{n}$ at time $t \in[0, T]$ consists of the values $x(t):=x\left(t ; 0, x_{0}\right)$ of all solutions $x:[0, t] \longrightarrow \mathbb{R}^{n}$ to $x^{\prime}=A x+B \eta+C v$ with $x(0)=x_{0} \in K(0)$ and any measurable noise $\eta(t) \in U(t)$ (e.g., [36, Ch. 9]). The key goal of OFC is to specify a control strategy $v=v(t, \tilde{K}) \in V$ in terms of the time $t \in[0, T]$ and a set-valued state estimation $\tilde{K} \subset \mathbb{R}^{n}$ which "for any starting position $\left(t_{0}, K_{0}\right), 0 \leq t_{0}<T$, would bring $x\left(T ; t_{0}, x_{0}\right) \in \mathbb{R}^{n}$ [of a solution $x$ with $x\left(t_{0}\right)=x_{0}$ ] to a preassigned neighborhood of the given target set $M \subset \mathbb{R}^{n}$ at given time $T$-whatever" the "uncertain item" $\eta(s) \in U(s)\left(s \in\left[t_{0}, t\right]\right)$ is [36, p. 375]. In terms of set-valued analysis, $K_{v}(t)$ is the reachable set of the initial set $K_{0} \subset \mathbb{R}^{n}$ and the differential inclusion $x^{\prime} \in A(s) x+B(s) U(s)+C(s) v(s)$ at time $t$ and, the OFC problem focuses a closed-loop control $v=v(t, \tilde{K})$ depending on a set $\tilde{K}$ of states (and not a state vector).

A relaxed OFC problem can be formulated as an optimal control problem: Minimize $\operatorname{dist}(K(T), M) \stackrel{\text { Def. }}{=} \sup _{\xi \in K(T)} \operatorname{dist}(\xi, M) \geq 0$ over all set-valued maps $K:[0, T] \rightsquigarrow$ $\mathbb{R}^{n}$ and measurable controls $v \in V$ such that each $K(t)$ coincides with the reachable set of $K_{0}$ and $x^{\prime} \in A(s) x+B(s) U(s)+C(s) v$ at time $t$. Indeed, if the minimum is attained and smaller than a given threshold $\rho>0$ then $K(T)$ is contained in the $\rho$-neighborhood of $M$ (as demanded originally).

This problem is already solved by KURZHANSKI and VARAIYA [35]—even with some generalizations. We mention it here because we consider it an excellent example of how useful sets (as states) instead of vectors can be for handling bounded (but) unknown perturbations deterministically. This is the first step to a broad class of optimization problems. Further examples of set-oriented descent methods with applications in image processing are discussed in, e.g., [22,39,40,66].

In general, reachable sets play an important role in deterministic systems with (bounded) "unknown noise" or other forms of lacking information (about initial states and parameters) because they are the smallest set containing all attainable states whatever the bounded uncertainties are (see, e.g., [10]). For nonlinear differential inclusions, several established numerical methods are related to grids (e.g., $[6,8,28,57])$ or based on the level-set formulation and its HJB equation (e.g., [38,45,46,65]). Hence, they are usually expensive and suffer from the "curse of dimensionality". For linear differential inclusions, the situation is different as the convexity of initial sets is obviously preserved. CHERNOUSKO, KURZHANSKI and others suggest ellipsoids for approximating these convex sets as supersets and subsets, respectively (see, e.g., [13$16,23,31,33,34,36])$. This special subclass of convex sets has the advantage that the evolution of an ellipsoid can be described in form of ordinary differential equations (ODEs) for its center and the underlying positive definite matrix. Hence, it reduces the numerical effort in high dimensions significantly. As the price to pay, however, this approach is usually applied to linear (or linearized) differential inclusions. For approx- 
imating solutions within any given collection of sets, QUINCAMPOIX and VELIOV suggest a general framework in [55] (but without a concrete numerical algorithm).

This article aims at a rather cheap numerical approximation algorithm for a larger class of set evolution problems: In comparison with KURZHANSKI's OFC problem, the closed-loop control $v=v(t, \tilde{K})$ has nonlinear influence on the state vector $x(t)$, i.e., the linear control equation $x^{\prime}=A(t) x+B(t) \eta+C(t) v$ (with bounded "unknown noise" $\eta(t) \in U(t))$ is replaced by $x^{\prime}=A(t, v) x+B(t, v) \eta$. Then, the same ansatz $v=v(t, \tilde{K})$ leads to a differential inclusion $x^{\prime}(t) \in \mathcal{A}(t, K(t)) x(t)+\mathcal{B}(t, K(t)) U(t)$ whose coefficient matrices depend on its own reachable set $K(t)$.

We extend the ellipsoidal approximation which KURZHANSKI et al. originally developed for linear differential inclusions. In particular, our sufficient conditions on the coefficients concern two aspects: Firstly, this set evolution problem is well posed. Secondly, the inclusion property is preserved, i.e., whenever $K(0)$ is contained in an ellipsoid $E(0)$, then each of them evolves independently of the other in such a way that $K(t) \subset E(t)$ holds for every $t$.

In the following, the well-posedness of the set evolution problem and the inclusion property of any two solutions are handled even for nonlinear differential inclusions and compact (not necessarily convex) sets. This part extends various results in, e.g., [3, $4,17-19,37,41,44,49,53,54,62]$. Then, in favor of fast numerical methods, the external approximation by ellipsoids is restricted to differential inclusions $x^{\prime} \in \mathcal{A}(\cdot, K) x+$ $\mathcal{B}(\cdot, K) U$ (i.e., linear in $x$ and $\eta \in U$ ). It is worth mentioning that its linear aspects do not concern the compact set $K(t) \subset \mathbb{R}^{n}$ or its set properties. We present sufficient conditions on the matrix coefficients such that the wanted set $K(t)$ is contained in the intersection of finitely many ellipsoids whose time-dependent centers and positive definite matrices solve an ODE system. The POMPEIU-HAUSDORFF distance between $K(t)$ and the intersection can be made arbitrarily small by choosing the numbers of ellipsoids sufficiently large. We also give an example that $K(t)$ might not have joint boundary points with each of the ellipsoids (as known in the classical case of linear inclusions, see Proposition 3.1 (2.) below).

This article is structured as follows. First, we summarize the notation used below. Section 2 specifies how the evolution of compact sets in time can be characterized in various (but equivalent) ways. It lays the foundations of what we call set evolution equations and, we give results about their initial value problems (IVP). In Sect. 3, we summarize the method by KURZHANSKI et al. for external ellipsoidal approximations of solutions to linear control systems. Then, it is extended to a new class of set evolution problems and, a nonlinear ODE system is suggested for any given number of ellipsoids whose intersections serve as approximations. Section 4 contains a numerical example. All proofs are collected in Sect. 5 .

Notation For any dimension, $n \in \mathbb{N}, \mathcal{K}\left(\mathbb{R}^{n}\right)$ denotes the set of all nonempty compact subsets of $\mathbb{R}^{n} . \mathcal{K}_{\mathrm{co}}\left(\mathbb{R}^{n}\right)$ abbreviates the set of all nonempty compact convex subsets of $\mathbb{R}^{n} \cdot\|\cdot\|$ is the EUCLIDean norm on $\mathbb{R}^{n},\|\cdot\|_{\text {op }}$ the related matrix norm. On the basis of the so-called POMPEIU-HAUSDORFF excess

$$
\mathbb{e}(A, B):=\sup _{x \in A} \operatorname{dist}(x, B) \stackrel{\text { Def. }}{=} \sup _{x \in A} \inf _{y \in B}\|x-y\| \quad\left(A, B \in \mathcal{K}\left(\mathbb{R}^{n}\right)\right),
$$


both $\mathcal{K}\left(\mathbb{R}^{n}\right)$ and its subset $\mathcal{K}_{\mathrm{co}}\left(\mathbb{R}^{n}\right)$ are usually supplied with the POMPEIU-HAUSDORFF metric

$$
\begin{aligned}
\mathbb{d}(A, B) & :=\max \{\mathbb{e}(A, B), \mathbb{e}(B, A)\} \\
& =\sup _{x \in \mathbb{R}^{n}}|\operatorname{dist}(x, A)-\operatorname{dist}(x, B)| \quad\left(A, B \in \mathcal{K}\left(\mathbb{R}^{n}\right)\right) .
\end{aligned}
$$

These metric spaces are known to be complete, locally compact and thus separable (e.g., $[4,7,30,58])$. The gap between $A, B \in \mathcal{K}\left(\mathbb{R}^{n}\right)$ is defined as $g(A, B):=$ $\inf _{x \in A} \operatorname{dist}(x, B) \stackrel{\text { Def. }}{=} \inf _{x \in A} \inf _{y \in B}\|x-y\|$.

Moreover, we use the same arrow $\rightsquigarrow$ for a set-valued (or multivalued) map as, e.g., [4,5], i.e., for any nonempty sets $Y, Z$ given, $g: Y \rightsquigarrow Z$ is a mapping relating each element $y \in Y$ to a subset $g(y) \subset Z$, which might consist of more than one element of $Z$.

A set-valued map is called a tube whenever it is defined on a subinterval of $\mathbb{R}$ and has nonempty set values. Further properties of its set values like compactness are usually mentioned explicitly (if required).

$\mathcal{L}^{n}$ denotes the LebesGue measure on $\mathbb{R}^{n}$. Set $\mathbb{B}_{R}:=\left\{x \in \mathbb{R}^{n} \mid\|x\|<R\right\}$ and $\overline{\mathbb{B}}_{R}:=\left\{x \in \mathbb{R}^{n} \mid\|x\| \leq R\right\}$ for $R \geq 0$. Solutions to ordinary differential equations or inclusions are usually understood in the sense of CARATHÉODORY (unless stated otherwise).

\section{Evolution Equations for Compact Subsets of $\mathbb{R}^{n}$}

\subsection{Reachable Sets Plus Feedback Lead to Set Evolution Equations}

Consider a control system $x^{\prime} \in g(t, x, U)$ (a.e.) where a function $g:[0, T] \times \mathbb{R}^{n} \times$ $\mathbb{R}^{m} \longrightarrow \mathbb{R}^{n}$ and a nonempty control subset $U \subset \mathbb{R}^{m}$ are given. The reachable set of an initial set $K_{0} \subset \mathbb{R}^{n}$ at time $t \in[0, T]$ is defined as

$$
\begin{aligned}
\mathcal{R}_{g(\cdot, \cdot, U)}\left(t, K_{0}\right):=\left\{x(t) \in \mathbb{R}^{n} \mid \begin{array}{l}
x:[0, t] \longrightarrow \mathbb{R}^{n} \text { absolutely continuous, } \\
\left.x^{\prime}(s) \in g(s, x(s), U) \text { for a.e. } s \in[0, t], \quad x(0) \in K_{0}\right\} .
\end{array}\right.
\end{aligned}
$$

Under appropriate assumptions about $g$ and $U$, each absolutely continuous solution $x:[0, t] \longrightarrow \mathbb{R}^{n}$ to the differential inclusion $x^{\prime} \in g(\cdot, x, U)$ is related to a LEBESGUE measurable control $u:[0, t] \longrightarrow U$ with $x^{\prime}(s)=g(s, x(s), u(s))$ for a.e. $s \in[0, t]$ due to well-known FiLIPPOV's selection theorem (e.g., [5, Theorem 8.2.10] or [30, Prop. II.2.25]). Hence, we prefer the focus on differential inclusions instead of ordinary differential equations with time-dependent control.

From the conceptual point of view, reachable sets can be interpreted as a way of "integrating" nonempty (usually closed) subsets w.r.t. time. In the special case, for example, that $g$ does not depend on the state vector $x \in \mathbb{R}^{n}$ explicitly, i.e., whenever $g=g(t, u)$, the reachable set $\mathcal{R}_{g}\left(t, K_{0}\right)$ can be expressed in terms of an AUMANN integral (w.r.t. $\left.\mathcal{L}^{1}\right) \mathcal{R}_{g(\cdot, U)}\left(t, K_{0}\right)=K_{0}+\int_{0}^{t} g(s, U) \mathrm{d} s$. (More details about this 
relationship between set integrals and reachable sets in terms of generalizations are in, e.g., $[40,42,43])$.

In the next step, we aim at an additional feedback w.r.t. the current compact subset of $\mathbb{R}^{n}$. This extension is motivated by KURZHANSKI's OFC problem (mentioned in the introduction) and by examples of descent methods in image segmentation, nonlocal agents-population interaction with closed-loop strategies and deterministic approaches to robust control problems (see, e.g., $[17,22,40,43,66])$. Assume that the right-hand side of the differential inclusion depends on a further argument, namely a nonempty compact subset of the state space $\mathbb{R}^{n}$, i.e., we consider the function $f$ : $[0, T] \times \mathbb{R}^{n} \times \mathcal{K}\left(\mathbb{R}^{n}\right) \times U \longrightarrow \mathbb{R}^{n}$ (with a fixed nonempty compact control set $U \subset \mathbb{R}^{m}$ ) instead of $g:[0, T] \times \mathbb{R}^{n} \times \mathbb{R}^{m} \longrightarrow \mathbb{R}^{n}$. Each compact-valued tube $K:[0, T] \rightsquigarrow \mathbb{R}^{n}$ (or, equivalently, every single-valued function $K:[0, T] \longrightarrow$ $\mathcal{K}\left(\mathbb{R}^{n}\right)$ ) leads to a nonautonomous differential inclusion $x^{\prime}(t) \in f(t, x(t), K(t), U)$ (a.e.) and its reachable set $\mathcal{R}_{f(\cdot, \cdot, K, U)}\left(t, K_{0}\right)$ at time $t \in[0, T]$. For $f, U$ and $K_{0} \in \mathcal{K}\left(\mathbb{R}^{n}\right)$ given, the problem is now to find a compact-valued tube $K(\cdot)$ satisfying $K(t)=\mathcal{R}_{f(\cdot,,, K, U)}\left(t, K_{0}\right)$ for every $t \in[0, T]$. In particular, the feedback mentioned previously concerns the differential inclusions which depends on $K(\cdot)$. Sufficient conditions of well-posedness and several examples are investigated in, e.g., [17].

\subsection{Differential Characterizations of These Compact-Valued Solutions}

Reachable sets of differential inclusions are also characterized by means of the so-called integral funnel equation. This approach is very popular among Russian mathematicians like FiliPPOVA, KuRZHANSKI, PANASYuK, TOLSTONOGOV and collaborators (e.g., [34,48,50-52,61,62] and related references).

Indeed, consider the compact initial set $K_{0} \subset \mathbb{R}^{n}$, the nonempty control set $U \subset \mathbb{R}^{m}$ and the function $g:[0, T] \times \mathbb{R}^{n} \times U \longrightarrow \mathbb{R}^{n}$ given. Under appropriate assumptions, the compact-valued tube of reachable sets $K:[0, T] \rightsquigarrow \mathbb{R}^{n}, t \mapsto K(t):=\mathcal{R}_{g(\cdot, \cdot, U)}\left(t, K_{0}\right)$ (in the sense of Eq. (1)) fulfills

$$
\lim _{h \downarrow 0} \frac{1}{h} \cdot \mathbb{d}\left(K(t+h), \bigcup_{x \in K(t)}(x+h \cdot g(t, x, U))\right)=0
$$

at a.e. time instant $t \in[0, T)$. Furthermore, slightly stronger hypotheses about $U$, $g$ even guarantee that it is the only LIPSCHITZ continuous compact-valued tube $K$ : $[0, T] \rightsquigarrow \mathbb{R}^{n}$ with $K(0)=K_{0}$ satisfying this integral funnel equation (2) for a.e. $t \in[0, T)$.

AUBIN suggests an alternative criterion of differential type and chooses it as the starting point of his so-called morphological equations (in the metric space $\left(\mathcal{K}\left(\mathbb{R}^{n}\right), \mathbb{d}\right)$ ) (see, e.g., [2-4]). At time instant $t \in[0, T)$ and for a short period $h>0$, it is now the reachable set $\mathcal{R}_{g(t, \cdot, U)}(h, K(t)) \subset \mathbb{R}^{n}$ of the autonomous differential inclusion $y^{\prime}(s) \in g(t, y(s), U)$ a.e. in $[0, h]$ which induces an approximation of $K(t+h) \subset \mathbb{R}^{n}$. Similarly to time derivatives of curves in a normed vector space, the distance between them is to vanish "in first order" for $h \downarrow 0$. 
In shape sensitivity analysis and shape optimization, the special case of $U \subset \mathbb{R}^{m}$ consisting of just a single vector leads to the so-called shape derivatives used by DELFOUR, SOKOOWSKI, ZOLÉSIO and others in the so-called velocity method (see, e.g., [20,21,59] and references therein).

In more detail, sufficient conditions on $U \subset \mathbb{R}^{m}$ and $g:[0, T] \times \mathbb{R}^{n} \times U \longrightarrow \mathbb{R}^{n}$ are known such that the tube $[0, T] \rightsquigarrow \mathbb{R}^{n}, t \mapsto \mathcal{R}_{g(\cdot, \cdot, U)}\left(t, K_{0}\right)$ of reachable sets (of the nonautonomous differential inclusion $x^{\prime} \in g(\cdot, x, U)$ ) is the only LIPSCHITZ continuous compact-valued tube $K:[0, T] \rightsquigarrow \mathbb{R}^{n}$ with $K(0)=K_{0} \in \mathcal{K}\left(\mathbb{R}^{n}\right)$ and

$$
\lim _{h \downarrow 0} \frac{1}{h} \cdot \mathbb{d}\left(K(t+h), \mathcal{R}_{g(t, \cdot, U)}(h, K(t))\right)=0 .
$$

Hence, we have three criteria on the reachable sets of a nonautonomous differential inclusion $x^{\prime} \in g(\cdot, x, U)$ and a compact initial set $K_{0} \subset \mathbb{R}^{n}$. Now we implement the additional aspect of set feedback (again) and obtain the following result about set evolution equations. Shortly speaking, it represents a special case of [42, Theorem 1] which concerns closed-valued tubes evolving along nonautonomous evolution inclusions in a separable BANACH space (instead of $\mathbb{R}^{n}$ ) and thus, we do not give a proof in detail.

Proposition 2.1 (Equivalent criteria for set evolution equations) Let $T>0, U \subset \mathbb{R}^{m}$ be nonempty compact and $f:[0, T] \times \mathbb{R}^{n} \times \mathcal{K}\left(\mathbb{R}^{n}\right) \times U \longrightarrow \mathbb{R}^{n}$ satisfy these conditions:

(i) For all $t \in[0, T], x \in \mathbb{R}^{n}$ and $M \in \mathcal{K}\left(\mathbb{R}^{n}\right)$, the set $f(t, x, M, U) \stackrel{\text { Def. }}{=}$ $\{f(t, x, M, u) \mid u \in U\} \subset \mathbb{R}^{n}$ is closed and convex.

(ii) (measurable in $t$ ) For all $x \in \mathbb{R}^{n}, M \in \mathcal{K}\left(\mathbb{R}^{n}\right)$ and $u \in U, f(\cdot, x, M, u)$ : $[0, T] \longrightarrow \mathbb{R}^{n}$ is measurable.

(iii) (continuous w.r.t. $u$ ) For all $t \in[0, T], x \in \mathbb{R}^{n}$ and $M \in \mathcal{K}\left(\mathbb{R}^{n}\right), f(t, x, M, \cdot)$ : $U \longrightarrow \mathbb{R}^{n}$ is continuous.

(iv) (LIPSCHITZ continuous w.r.t. $x$ ) There is $\lambda \geq 0$ such that for all $t \in[0, T], u \in U$ and $M \in \mathcal{K}\left(\mathbb{R}^{n}\right), f(t, \cdot, M, u): \mathbb{R}^{n} \longrightarrow \mathbb{R}^{n}$ is $\lambda$-LIPSCHITZ continuous.

(v) (continuous w.r.t. the compact set) For all $x \in \mathbb{R}^{n}, u \in U$ and a.e. $t \in[0, T]$, the function $f(t, x, \cdot, u):\left(\mathcal{K}\left(\mathbb{R}^{n}\right), \mathbb{d}\right) \longrightarrow \mathbb{R}^{n}$ is continuous.

(vi) (uniform linear growth w.r.t. $x$ only) There is $\Gamma \geq 0$ such that $\|f(t, x, M, u)\| \leq$ $\Gamma(1+\|x\|)$ for all $t \in[0, T], x \in \mathbb{R}^{n}, M \in \mathcal{K}\left(\mathbb{R}^{n}\right)$ and $u \in U$.

Then, the following statements are equivalent for every compact-valued tube $K$ : $[0, T] \rightsquigarrow \mathbb{R}^{n}$ :

(1.) At every time $t \in[0, T], K(t) \subset \mathbb{R}^{n}$ coincides with the simultaneous reachable set of $K(0) \subset \mathbb{R}^{n}$ and the nonautonomous differential inclusion $x^{\prime}(s) \in$ $f(s, x(s), K(s), U)$ for a.e. $s \in[0, t]$.

(2.) $K(\cdot)$ is LIPSCHITZ (w.r.t. d) and $\lim _{h \downarrow 0} \frac{1}{h} \cdot \mathbb{d}\left(K(t+h), \bigcup_{x \in K(t)}(x+h\right.$. $f(t, x, K(t), U)))=0$ for a.e. $t$. 
(3.) $K(\cdot)$ is LiPSCHITZ (w.r.t. $\mathbb{d})$ and fulfills $\lim _{h \downarrow 0} \frac{1}{h} \cdot \mathbb{d}\left(K(t+h), \mathcal{R}_{f(t, \cdot, K(t), U)}\right.$ $(h, K(t)))=0$ for a.e. $t$ where $\mathcal{R}_{f(t, \cdot, K(t), U)}(h, K(t)) \subset \mathbb{R}^{n}$ denotes the reachable set of the initial set $K(t)$ and the autonomous differential inclusion $y^{\prime} \in f(t, y(\cdot), K(t), U)$ a.e. in $[0, h]$ at time $h \geq 0$.

\subsection{Compact-Valued Solutions to the Initial Value Problem}

On the search for solutions to the corresponding IVP without state constraints, standard ODE methods like EULER method or successive approximation can be adapted to the compact-valued setting. An additional assumption of LIPSCHITZ continuity (w.r.t. the set argument) proves to be sufficient for extending the CAUCHY-LIPSCHITZ theorem about both existence and uniqueness. Various theorems about this topic can be found in references like $[3,4,17,19,37,41,44,53,62]$.

The following statement is a reformulation of [17, Theorem 4.2] under the slightly stronger assumptions that the linear growth condition and the LIPSCHITZ constants are uniform w.r.t. $x$ here (see also, e.g., [4, Theorem 4.1.2], [41, Theorem 1.72], [62, § 1]).

Proposition 2.2 (Existence and uniqueness of solution tubes) In addition to the assumptions of Proposition 2.1 about the control set $U \subset \mathbb{R}^{m}$ and the function $f$ : $[0, T] \times \mathbb{R}^{n} \times \mathcal{K}\left(\mathbb{R}^{n}\right) \times U \longrightarrow \mathbb{R}^{n}$, suppose the following condition:

$v^{\prime}$ (LIPSCHITZ continuous w.r.t. the compact set) There exists $\Lambda \in L^{1}([0, T])$ such that for all $x \in \mathbb{R}^{n}, u \in U$ and a.e. $t \in[0, T], f(t, x, \cdot, u):\left(\mathcal{K}\left(\mathbb{R}^{n}\right), \mathbb{d}\right) \longrightarrow \mathbb{R}^{n}$ is $\Lambda(t)$-LIPSCHITZ continuous.

Then for every $K_{0} \in \mathcal{K}\left(\mathbb{R}^{n}\right)$, there exists a unique compact-valued tube $K:[0, T] \rightsquigarrow$ $\mathbb{R}^{n}$ with $K(0)=K_{0}$ satisfying the three equivalent conditions $2.1(1)-.(3$.$) .$

Definition 2.3 Let $K_{0} \in \mathcal{K}\left(\mathbb{R}^{n}\right)$ and $f:[0, T] \times \mathbb{R}^{n} \times \mathcal{K}\left(\mathbb{R}^{n}\right) \times U \longrightarrow \mathbb{R}^{n}$ be given as in Proposition 2.2. The compact-valued tube $K:[0, T] \rightsquigarrow \mathbb{R}^{n}$ specified there uniquely is called the solution tube of the IVP $\stackrel{\circ}{K}(t)=f(t, \cdot, K(t), U)$ in $[0, T], K(0)=K_{0}$.

Remark 2.4 From now on, we do not really distinguish between two established concepts, i.e., quasidifferential equations by PANASYUK applied to $\left(\mathcal{K}\left(\mathbb{R}^{n}\right), \mathbb{d}\right)$ (e.g., $[49,53,54])$ and morphological equations by AUBIN which are the example of his mutational equations applied to $\left(\mathcal{K}\left(\mathbb{R}^{n}\right), \mathbb{d}\right)$ and the "transitions" induced by reachable sets (see, e.g., [3,4,41]). Indeed, Proposition 2.1 states their equivalence under suitable assumptions.

Several publications characterize the solution to a set evolution equation in terms of the HUKUHARA derivative (w.r.t. time), and thus, the tubes are always assumed to be convex-valued (see, e.g., [37,44,62] and related references). Under suitable assumptions about $\tilde{f}:[0, T] \times \mathcal{K}\left(\mathbb{R}^{n}\right) \times U \longrightarrow \mathbb{R}^{n}$, a convex-valued tube $K:[0, T] \rightsquigarrow \mathbb{R}^{n}$ "solves" the differential equation $D_{H} K(t)=\widetilde{f}(t, K(t), U)$ (in that sense) if and only if it fulfills the following condition on AUMANN integrals for every $t \in[0, T]$

$$
K(t)=K(0)+\int_{0}^{t} \tilde{f}(s, K(s), U) \mathrm{d} s .
$$


As a consequence, we consider that concept as the special case of our approach in which the function $f:[0, T] \times \mathbb{R}^{n} \times \mathcal{K}\left(\mathbb{R}^{n}\right) \times U \longrightarrow \mathbb{R}^{n}$ does not depend on the state $x \in \mathbb{R}^{n}$ explicitly. Indeed, the right-hand side in Eq. (4) coincides with the reachable set of $K(0) \subset \mathbb{R}^{n}$ and $y^{\prime} \in \widetilde{f}(\cdot, K(\cdot), U)$ at time $t$ (as mentioned in Sect. 2.1).

It is worth mentioning that even in the autonomous linear case, state $x \in \mathbb{R}^{n}$ and set $K(s) \subset \mathbb{R}^{n}$ cannot be simply exchanged with each other. Indeed, TOLSTONOGOV gives the example in $\mathbb{R}[62$, p. $209 \mathrm{f}$.] that the reachable interval of the single initial state $0 \in \mathbb{R}$ and $x^{\prime} \in-\alpha x+U$ (with $\alpha>0$ and $U=-U \subset \mathbb{R}$ having more than one element) does not coincide with the solution $K(t)$ of $K(t)=\int_{0}^{t}(-\alpha K(s)+U) \mathrm{d} s$.

Proposition 2.5 (Continuous dependence on data) Let $U \subset \mathbb{R}^{m}$ and $f_{1}, f_{2}:[0, T] \times$ $\mathbb{R}^{n} \times \mathcal{K}\left(\mathbb{R}^{n}\right) \times U \longrightarrow \mathbb{R}^{n}$ fulfill the assumptions of Proposition 2.2 (with the same $\Lambda \in L^{1}([0, T])$ and $\left.\lambda, \Gamma \geq 0\right)$.

Then for any initial sets $K_{0}, M_{0} \in \mathcal{K}\left(\mathbb{R}^{n}\right)$ with $K_{0} \cup M_{0} \subset \overline{\mathbb{B}}_{r}$, the respective solution tubes $K, M:[0, T] \rightsquigarrow \mathbb{R}^{n}$ satisfy the following a priori bound with $R:=(r+\Gamma T) e^{\Gamma T}$ at every time $t \in[0, T]$

$$
\begin{aligned}
\mathbb{d}(K(t), M(t)) \leq & \mathbb{d}\left(K_{0}, M_{0}\right) \cdot e^{\int_{0}^{t}(\Lambda+\lambda) \mathrm{d} s} \\
& +\int_{0}^{t} \sup _{x \in \overline{\mathbb{B}}_{R}} \mathbb{d}\left(f_{1}(s, x, M(s), U), f_{2}(s, x, M(s), U)\right) \cdot e^{\int_{s}^{t}(\Lambda+\lambda) \mathrm{d} \tau} \mathrm{d} s .
\end{aligned}
$$

\subsection{The Inclusion Principle for Set Evolution Equations}

Now we focus on sufficient conditions on the coefficients such that $K(0) \subset M(0) \cap$ $\widetilde{M}(0) \subset \mathbb{R}^{n}$ always implies $K(t) \subset M(t) \cap \widetilde{M}(t)$ at each time $t \in[0, T]$ for the respective solutions $K, M, \widetilde{M}:[0, T] \rightsquigarrow \mathbb{R}^{n}$. The following result extends [4, Theorem 4.3.3] to nonautonomous set evolution equations whose functions on the right-hand side are just measurable w.r.t. time.

Definition 2.6 [5, Definition 4.1.5] Let $K \subset \mathbb{R}^{n}$ be nonempty and $x \in \bar{K}$. The intermediate cone or adjacent cone $T_{K}^{b}(x)$ of $K$ at $x$ is defined as $T_{K}^{b}(x):=\{v \in$ $\left.\mathbb{R}^{n} \mid \lim _{h \downarrow 0} \frac{1}{h} \cdot \operatorname{dist}(x+h v, K)=0\right\}$.

Proposition 2.7 (Inclusion principle of solution tubes) Let $T>0, U \subset \mathbb{R}^{m}$ and $f, g$, $\tilde{g}:[0, T] \times \mathbb{R}^{n} \times \mathcal{K}\left(\mathbb{R}^{n}\right) \times U \longrightarrow \mathbb{R}^{n}$ satisfy the hypotheses of Proposition 2.1 and

(v') There exists $\Lambda \geq 0$ such that $f(t, x, \cdot, u), g(t, x, \cdot, u), \widetilde{g}(t, x, \cdot, u):\left(\mathcal{K}\left(\mathbb{R}^{n}\right)\right.$, d) $\longrightarrow \mathbb{R}^{n}$ are $\Lambda$-LIPSCHITZ continuous for all $x \in \mathbb{R}^{n}, u \in U$ and $t \in[0, T]$.

(vii') For a.e. $t \in[0, T]$ and all $x \in \mathbb{R}^{n}, K, M_{1}, M_{2} \in \mathcal{K}\left(\mathbb{R}^{n}\right)$ with $x \in K \subset M_{1} \cap M_{2}$, it holds

$$
f(t, x, K, U) \subset\left(g\left(t, x, M_{1}, U\right)+T_{M_{1}}^{b}(x)\right) \cap\left(\widetilde{g}\left(t, x, M_{2}, U\right)+T_{M_{2}}^{b}(x)\right) .
$$


For all initial $K_{0}, M_{0}, \tilde{M}_{0} \in \mathcal{K}\left(\mathbb{R}^{n}\right)$ with $K_{0} \subset M_{0} \cap \tilde{M}_{0}$, the solution tubes $K, M$, $\widetilde{M}:[0, T] \rightsquigarrow \mathbb{R}^{n}$ of

$$
\left\{\begin{array} { l } 
{ \stackrel { \circ } { K } ( t ) = f ( t , \cdot , K ( t ) , U ) } \\
{ K ( 0 ) = K _ { 0 } }
\end{array} \quad \left\{\begin{array} { l } 
{ \stackrel { \circ } { M } ( t ) = g ( t , \cdot , M ( t ) , U ) } \\
{ M ( 0 ) = M _ { 0 } }
\end{array} \quad \left\{\begin{array}{l}
\stackrel{e}{M}(t)=\tilde{g}(t, \cdot, \tilde{M}(t), U) \\
\widetilde{M}(0)=\widetilde{M}_{0}
\end{array}\right.\right.\right.
$$

respectively, satisfy $K(t) \subset M(t) \cap \tilde{M}(t)$ for every $t \in[0, T]$.

\section{Ellipsoidal Approach to External Approximations}

\subsection{Ellipsoidal Approximations of Reachable Sets for Linear Control Systems}

For several decades, ellipsoids have been very popular for approximating convex compact subsets of $\mathbb{R}^{n}$. Russian mathematicians, in particular, like CHERNOUSKO, FILIPPOVA, KURZHANSKI and collaborators have proposed them for estimating reachable sets of control systems that are usually linear or linearized (see, e.g., [13$16,23,31,33,36]$ and related references).

Their key advantage is the simple algebraic characterization: Each (so-called nondegenerate) ellipsoid is determined completely by its center $p \in \mathbb{R}^{n}$ and its matrix $Q \in \mathbb{R}^{n \times n}$ (symmetric and positive definite)

$$
\mathcal{E}(p, Q):=\left\{x \in \mathbb{R}^{n} \mid(x-p)^{\top} Q^{-1}(x-p) \leq 1\right\} .
$$

We start with a linear time-variant control system $x^{\prime} \in A(\cdot) x+B(\cdot) U$ (a.e. in $[0, T]$ ) with $A:[0, T] \longrightarrow \mathbb{R}^{n \times n}$ and $B:[0, T] \longrightarrow \mathbb{R}^{n \times m}$ given (as in, e.g., $[33,36])$. For the sake of simplicity, the ellipsoidal control set $U:=\mathcal{E}\left(q_{u}, Q_{u}\right) \subset \mathbb{R}^{m}$ does not depend on time. For each initial $K_{0} \in \mathcal{K}\left(\mathbb{R}^{n}\right)$, the tube $K:[0, T] \rightsquigarrow \mathbb{R}^{n}$ of reachable sets is characterized by the integral funnel equation

$$
0=\lim _{h \downarrow 0} \frac{1}{h} \cdot \mathbb{d}\left(K(t+h),(\mathbb{1}+h A(t)) K(t)+h B(t) \mathcal{E}\left(q_{u}, Q_{u}\right)\right) .
$$

In general, the reachable set $K(t) \subset \mathbb{R}^{n}$ is not an ellipsoid though-even if $K_{0}$ is one.

For characterizing an approximating ellipsoid-valued tube $E:[0, T] \rightsquigarrow \mathbb{R}^{n}$, KURZHANSKI et al. suggest the weaker criterion (5) below based on the POMPEIUHAUSDORFF excess $\mathbb{e}$ instead of the metric $\mathbb{d}$ (see, e.g., [33, § 3.3], [36, § 3.4]). It leads to the following results reformulating [36, Theorems 3.4.1, 3.4.2]. Its minimal property (2.) (c) indicates in which sense these ellipsoids are "optimal approximations" of $\mathcal{R}_{A \cdot+B U}\left(t, K_{0}\right)$ within their class.

Proposition $3.1[36,63]$ Let $A:[0, T] \longrightarrow \mathbb{R}^{n \times n}$ and $B:[0, T] \longrightarrow \mathbb{R}^{n \times m}$ be continuous. $G:\left\{(t, s) \in[0, T]^{2} \mid t \geq s\right\} \longrightarrow \mathbb{R}^{n \times n}$ denotes the fundamental matrix of $x^{\prime}=A(t) x$. Suppose that the initial set $K_{0}:=\mathcal{E}\left(x_{0}, X_{0}\right) \in \mathcal{K}\left(\mathbb{R}^{n}\right)$ and the control set $U:=\mathcal{E}\left(q_{u}, Q_{u}\right) \subset \mathbb{R}^{m}$ are non-degenerate.

Then, the following statements hold: 
(1.) If an ellipsoid-valued tube $E:[0, T] \rightsquigarrow \mathbb{R}^{n}$ is LIPSCHITZ continuous with $E(0)=$ $K_{0}$ and

$$
0=\lim _{h \downarrow 0} \frac{1}{h} \cdot \mathbb{e}\left((\mathbb{1}+h A(t)) E(t)+h B(t) \mathcal{E}\left(q_{u}, Q_{u}\right), \quad E(t+h)\right)
$$

for a.e. $t \in[0, T]$, then the reachable set $\mathcal{R}_{A+B U}\left(t, K_{0}\right) \subset \mathbb{R}^{n}$ of $x^{\prime} \in A(\cdot) x+$ $B(\cdot) U$ is contained in $E(t)$ at each time $t \in[0, T]$.

(2.) In addition, assume that the control system $x^{\prime} \in A(\cdot) x+B(\cdot) U$ (a.e.) is completely controllable. For $\ell_{0} \in \mathbb{R}^{n}$ fixed arbitrarily, consider $\ell:[0, T] \longrightarrow \mathbb{R}^{n}$, $t \longmapsto G(0, t)^{\top} \ell_{0}$ and let $x:[0, T] \longrightarrow \mathbb{R}^{n}, X:[0, T] \longrightarrow \mathbb{R}^{n \times n}$ denote the unique solutions of IVP

$$
\begin{array}{ll}
x^{\prime}(t)=A(t) \quad x(t)+B(t) q_{u}, & x(0)=x_{0} \\
X^{\prime}(t)=A(t) X(t)+X(t) A(t)^{\top}+\pi(t) X(t)+\frac{1}{\pi(t)} Q_{B}(t), & X(0)=X_{0}
\end{array}
$$

with $Q_{B}(t):=B(t) Q_{u} B(t)^{\top} \in \mathbb{R}^{n \times n}, \pi(t):=\sqrt{\frac{\left\langle\ell(t), Q_{B}(t) \ell(t)\right\rangle}{\langle\ell(t), X(t) \ell(t)\rangle}}>0$.

Then, the ellipsoid-valued tube $E:[0, T] \rightsquigarrow \mathbb{R}^{n}, t \mapsto \mathcal{E}(x(t), X(t))$ has these properties:

a E satisfies the limit condition (5) for each $t \in[0, T)$.

$b$ For each $t, E(t)$ is an external approximation of $\mathcal{R}_{A \cdot+B U}\left(t, K_{0}\right)$, i.e., $\mathcal{R}_{A \cdot+B U}\left(t, K_{0}\right) \subset E(t)$.

$c E$ is minimal in the class of ellipsoids w.r.t. set inclusions, i.e., for every $t \in$ $[0, T]$, there does not exist any ellipsoid $\widetilde{\widetilde{E}} \subset \mathbb{R}^{n}$ with $\mathcal{R}_{A \cdot+B U}\left(t, K_{0}\right) \subset \widetilde{E} \varsubsetneqq$ $E(t)$.

$d$ For every $t \in[0, T], \mathcal{R}_{A+B U}\left(t, K_{0}\right)$ and $E(t)$ have a boundary point $\xi(t)$ in common such that $\ell(t)$ is normal to both sets in $\xi(t)$, i.e., $\xi(t)=x(t)+$ $\langle\ell(t), X(t) \ell(t)\rangle^{-\frac{1}{2}} X(t) \ell(t)$.

\subsection{Some External Approximation for a Solution to a Set Evolution Equation}

Now we aim to extend these results from linear time-variant control systems (and their reachable sets) to a class of set evolution equations (and their solution tubes in the sense of Definition 2.3).

Hence, the right-hand side of the set evolution equation (described by $f$ in Sect. 2.2) is now supposed to be linear w.r.t. $x$ and $u$. Reachable sets of nonautonomous linear differential inclusions are known to be always convex as a consequence of the variations of constants formula. Thus, we focus on convex compact subsets of $\mathbb{R}^{n}$ instead of $\mathcal{K}\left(\mathbb{R}^{n}\right)$. In analogy to the notation in Sect. 3.1, let the coefficient functions

$$
\mathcal{A}:[0, T] \times \mathcal{K}_{\mathrm{co}}\left(\mathbb{R}^{n}\right) \longrightarrow \mathbb{R}^{n \times n}, \quad \mathcal{B}:[0, T] \times \mathcal{K}_{\mathrm{co}}\left(\mathbb{R}^{n}\right) \longrightarrow \mathbb{R}^{n \times m}
$$


be given. We consider the set evolution equation with the function

$$
\begin{aligned}
& f:[0, T] \times \mathbb{R}^{n} \times \mathcal{K}\left(\mathbb{R}^{n}\right) \times U \longrightarrow \mathbb{R}^{n}, \quad(t, x, M, u) \\
& \quad \longmapsto \mathcal{A}(t, \overline{\operatorname{co}} M) x+\mathcal{B}(t, \overline{\operatorname{co}} M) u .
\end{aligned}
$$

Proposition 3.2 Let $\mathcal{A}:[0, T] \times \mathcal{K}_{c o}\left(\mathbb{R}^{n}\right) \longrightarrow \mathbb{R}^{n \times n}, \mathcal{B}:[0, T] \times \mathcal{K}_{c o}\left(\mathbb{R}^{n}\right) \longrightarrow \mathbb{R}^{n \times m}$ and $U \subset \mathbb{R}^{m}$ satisfy the following conditions:

(i) $U:=\mathcal{E}\left(q_{u}, Q_{u}\right) \subset \mathbb{R}^{m}$ is non-degenerate.

(ii) For every $M \in \mathcal{K}_{c o}\left(\mathbb{R}^{n}\right), \mathcal{A}(\cdot, M):[0, T] \longrightarrow \mathbb{R}^{n \times n}$ and $\mathcal{B}(\cdot, M):[0, T] \longrightarrow$ $\mathbb{R}^{n \times m}$ are measurable.

(iii) There is $\Lambda \geq 0$ such that $\mathcal{A}(t, \cdot):\left(\mathcal{K}_{c o}\left(\mathbb{R}^{n}\right), \mathbb{d}\right) \longrightarrow \mathbb{R}^{n \times n}$ and $\mathcal{B}(t, \cdot)$ : $\left(\mathcal{K}_{c o}\left(\mathbb{R}^{n}\right), \mathbb{d}\right) \longrightarrow \mathbb{R}^{n \times m}$ are $\Lambda$-LIPSCHITZ continuous for every $t \in[0, T]$.

(iv) There is $\Gamma \geq 0$ such that for all $t \in[0, T]$ and $M \in \mathcal{K}_{c o}\left(\mathbb{R}^{n}\right),\|\mathcal{A}(t, M)\|_{\text {op}}$, $\sup _{u \in U}\|\mathcal{B}(t, M) u\| \leq \Gamma$.

(v) For a.e. $t \in[0, T]$ and all $x \in \mathbb{R}^{n}, K, M \in \mathcal{K}_{c o}\left(\mathbb{R}^{n}\right)$ with $x \in K \subset M$,

$$
\mathcal{A}(t, K) x+\mathcal{B}(t, K) U \subset \mathcal{A}(t, M) \quad x+\mathcal{B}(t, M) \quad U+T_{M}^{b}(x) .
$$

Consider $K_{0} \in \mathcal{K}_{c o}\left(\mathbb{R}^{n}\right)$ with nonempty interior. Assume for each of the ellipsoidvalued tubes $E_{1}, \ldots, E_{N}:[0, T] \rightsquigarrow \mathbb{R}^{n}$ and $\mathcal{E}_{\cap}(t):=\bigcap_{k=1}^{N} E_{k}(t):$

(vi) $E_{j}(\cdot)$ is LIPSCHITZ continuous with $K_{0} \subset E_{j}(0)$.

(vii) For a.e. $t \in[0, T), \quad 0=\lim _{h \downarrow 0} \frac{1}{h} \cdot \mathbb{e}\left(\left(\mathbb{1}+h \mathcal{A}\left(t, \mathcal{E}_{\cap}(t)\right)\right) \quad E_{j}(t)+\right.$

$$
\left.h \mathcal{B}\left(t, \mathcal{E}_{\cap}(t)\right) U, \quad E_{j}(t+h)\right) .
$$

Then, the unique solution tube $K:[0, T] \rightsquigarrow \mathbb{R}^{n}$ of the IVP

$$
\stackrel{\circ}{K}(t)=\mathcal{A}(t, K(t)) x+\mathcal{B}(t, K(t)) U \text { in }[0, T], \quad K(0)=K_{0}
$$

fulfills $K(t) \subset \mathcal{E}_{\cap}(t)$ for every $t \in[0, T]$.

\subsection{A Computational Method for an External Approximation With Ellipsoidal Values}

Proposition 3.1 (2.) provides an ODE system which specifies an ellipsoid-valued tube as an external approximation of the reachable set. In more detail, it concerns the reachable set $\mathcal{R}_{A+B U}\left(t, K_{0}\right)$ of a nonautonomous linear differential inclusion $x^{\prime} \in$ $A(t) x+B(t) U$ and, the ODE system describes the evolution of the center $x(t) \in$ $\mathbb{R}^{n}$ and the positive definite symmetric matrix $X(t) \in \mathbb{R}^{n \times n}$ of the time-dependent ellipsoids.

Set evolution equations are essentially based on the notion that the coefficients depend on the current set in addition: $\mathcal{A}=\mathcal{A}(t, M), \mathcal{B}=\mathcal{B}(t, M)$ instead of $A=A(t)$ and 
$B=B(t)$, respectively. This gist motivates us to consider the following nonlinear ODE system

$$
\begin{aligned}
& \ell^{\prime}(t)=-\mathcal{A}(t, \mathcal{E}(x(t), X(t)))^{\top} \quad \ell(t), \\
& x^{\prime}(t)=\mathcal{A}(t, \mathcal{E}(x(t), X(t))) \\
& X^{\prime}(t)=\mathcal{A}(t, \mathcal{E}(x(t), X(t))) \\
& \quad+\frac{1}{\pi(t)} Q_{\mathcal{B}}(t, x(t)+X(t), X(t))
\end{aligned}
$$

with the abbreviations $Q_{\mathcal{B}}(t, x(t), X(t))=\mathcal{B}(t, \mathcal{E}(x(t), X(t))) Q_{u} \mathcal{B}(t, \mathcal{E}(x(t)$, $X(t)))^{\top} \in \mathbb{R}^{n \times n}$ and $\pi(t)=\sqrt{\frac{\left\langle\ell(t), Q_{\mathcal{B}}(t, x(t), X(t)) \ell(t)\right\rangle}{\langle\ell(t), X(t) \ell(t)\rangle}}$.

Strictly speaking, Proposition 3.2 considers the intersection of finitely many ellipsoids as an external approximation of the solution value $K(t) \subset \mathbb{R}^{n}$. Assumption 3.2 (vii) indicates how to choose the coefficients appropriately, i.e., in terms of their pointwise intersection $\mathcal{E}_{\cap}(t)$. It leads directly to ODE system (7) below which is easy to solve numerically (using standard methods for the support function of $\mathcal{E}_{\cap}(t)$ ).

Proposition 3.3 Let $\mathcal{A}:[0, T] \times \mathcal{K}_{c o}\left(\mathbb{R}^{n}\right) \longrightarrow \mathbb{R}^{n \times n}, \mathcal{B}:[0, T] \times \mathcal{K}_{c o}\left(\mathbb{R}^{n}\right) \longrightarrow \mathbb{R}^{n \times m}$ and $U=\mathcal{E}\left(q_{u}, Q_{u}\right) \subset \mathbb{R}^{m}$ satisfy the Assumptions $3.2(i)$, (iii) and

(ii') For every $M \in \mathcal{K}_{c o}\left(\mathbb{R}^{n}\right), \mathcal{A}(\cdot, M):[0, T] \longrightarrow \mathbb{R}^{n \times n}$ and $\mathcal{B}(\cdot, M):[0, T] \longrightarrow$ $\mathbb{R}^{n \times m}$ are continuous.

(iv') There exists $\Gamma \geq 0$ such that for all $t \in[0, T]$ and $M \in \mathcal{K}_{c o}\left(\mathbb{R}^{n}\right)$,

$$
\begin{aligned}
& \max \left\{\|\mathcal{A}(t, M)\|_{\text {op }},\left\|\mathcal{A}(t, M)^{\top}\right\|_{\mathrm{op}},\right. \\
& \left.\sup _{u \in U}\|\mathcal{B}(t, M) u\|,\|\mathcal{B}(t, M)\|_{\mathrm{op}},\left\|\mathcal{B}(t, M)^{\top}\right\|_{\mathrm{op}}\right\} \leq \Gamma .
\end{aligned}
$$

(vi') $m=n$ and $\mathcal{B}(t, M) \in \mathbb{R}^{n \times n}$ is invertible, $\left\|\left(\mathcal{B}(t, M)^{\top}\right)^{-1}\right\|_{\mathrm{op}} \leq \Gamma$ for all $t \in[0, T], M \in \mathcal{K}_{c o}\left(\mathbb{R}^{n}\right)$.

For $j=1, \ldots, N$, let $\ell_{0 j} \in \mathbb{R}^{n} \backslash\{0\}, x_{0 j} \in \mathbb{R}^{n}$ and positive definite symmetric $X_{0 j} \in \mathbb{R}^{n \times n}$ be given such that $\bigcap_{k=1}^{N} \mathcal{E}\left(x_{0 k}, X_{0 k}\right) \subset \mathbb{R}^{n}$ has nonempty interior. Consider the ODE system

$$
\begin{aligned}
\ell_{j}^{\prime}(t)= & -\mathcal{A}\left(t, \mathcal{E}_{\cap}(t)\right)^{\top} \quad \ell_{j}(t) \\
x_{j}^{\prime}(t)= & \mathcal{A}\left(t, \mathcal{E}_{\cap}(t)\right) \quad x_{j}(t)+\mathcal{B}\left(t, \mathcal{E}_{\cap}(t)\right) q_{u}, \\
X_{j}^{\prime}(t)= & \mathcal{A}\left(t, \mathcal{E}_{\cap}(t)\right) X_{j}(t)+\pi_{j}(t) X_{j}(t)+X_{j}(t) \mathcal{A}\left(t, \mathcal{E}_{\cap}(t)\right)^{\top} \\
& \quad+\frac{1}{\pi_{j}(t)} Q_{\mathcal{B} \cap}(t)
\end{aligned}
$$

with the abbreviations

$$
\begin{aligned}
& \mathcal{E}_{\cap}(t):=\bigcap_{k=1}^{N} \mathcal{E}\left(x_{k}(t), X_{k}(t)\right) \in \mathcal{K}_{c o}\left(\mathbb{R}^{n}\right), \\
& Q_{\mathcal{B} \cap}(t):=\mathcal{B}\left(t, \mathcal{E}_{\cap}(t)\right) Q_{u} \mathcal{B}\left(t, \mathcal{E}_{\cap}(t)\right)^{\top} \in \mathbb{R}^{n \times n}, \pi_{j}(t):=\sqrt{\frac{\left\langle\ell_{j}(t), Q_{\mathcal{B} \cap}(t) \ell_{j}(t)\right\rangle}{\left\langle\ell_{j}(t), X_{j}(t) \ell_{j}(t)\right\rangle}} .
\end{aligned}
$$

Then, the following statements hold: 
(1.) There exist unique solutions $\ell_{j}, x_{j}:[0, T] \longrightarrow \mathbb{R}^{n}, X_{j}:[0, T] \longrightarrow \mathbb{R}^{n \times n}$ to ODE system (7) with the initial values $\ell_{0 j}, x_{0 j} \in \mathbb{R}^{n}, X_{0 j} \in \mathbb{R}^{n \times n}$, respectively $(j=1, \ldots, N)$. Moreover, for all $t \in[0, T]$ :

- $\mathcal{E}_{\cap}(t)$ has nonempty interior.

- $\ell_{j}(t) \neq 0$ and $X_{j}(t) \in \mathbb{R}^{n \times n}$ is symmetric.

- There are $\widetilde{c}_{j}, \widetilde{C}_{j}>0$ (depending on $\Gamma, Q_{u}$ ) and $c_{j}, C_{j}>0$ (depending on $\left.\Gamma, Q_{u}, X_{0 j}\right)$ with

$$
\begin{aligned}
\left\|\ell_{0 j}\right\|_{2}^{2} \cdot \tilde{c}_{j} e^{-2 \Gamma t} & \leq\left\langle\ell_{j}(t), Q_{\mathcal{B} \cap}(t) \ell_{j}(t)\right\rangle & & \leq \widetilde{C}_{j} e^{2 \Gamma t}\left\|\ell_{0 j}\right\|^{2} \\
c_{j} e^{-2 \Gamma t} & \leq \pi_{j}(t) & & \leq C_{j} e^{2 \Gamma t} \\
\|v\|^{2} \cdot c_{j} e^{-2 \Gamma t} & \leq\left\langle v, X_{j}(t) v\right\rangle & & \text { for all } v \in \mathbb{R}^{n} .
\end{aligned}
$$

(2.) Consider solutions $\ell_{j}(\cdot), x_{j}(\cdot), X_{j}(\cdot)(j=1, \ldots, N)$ as characterized in statement (1.). Then, each tube $E_{j}:[0, T] \rightsquigarrow \mathbb{R}^{n}, t \mapsto \mathcal{E}\left(x_{j}(t), X_{j}(t)\right)$ $(j=1, \ldots, N)$ is LIPSCHITZ and satisfies for every $t$

$$
0=\lim _{h \downarrow 0} \frac{1}{h} \cdot \mathbb{e}\left(\left(\mathbb{1}+h \mathcal{A}\left(t, \mathcal{E}_{\cap}(t)\right)\right) E_{j}(t)+h \mathcal{B}\left(t, \mathcal{E}_{\cap}(t)\right) U, \quad E_{j}(t+h)\right) .
$$

In regard to external approximations for set evolutions, Proposition 3.2 has the direct consequence:

Corollary 3.4 Let the initial set $K_{0}=\mathcal{E}\left(x_{0}, X_{0}\right) \subset \mathbb{R}^{n}$ be given. Under the assumptions of Proposition 3.3, suppose $\ell_{j}(\cdot), x_{j}(\cdot), X_{j}(\cdot)(j=1, \ldots, N)$ are solutions to ODE system (7) with $\ell_{j}(0) \in \mathbb{R}^{n} \backslash\{0\}, x_{j}(0)=x_{0}, X_{j}(0)=X_{0}$. Furthermore assume condition 3.2(v).

Then, the unique solution $K:[0, T] \rightsquigarrow \mathbb{R}^{n}$ of IVP (6) fulfills $K(t) \subset \mathcal{E}_{\cap}(t)$ for every $t$.

\subsection{No Minimal Property of This Ellipsoidal Approximation in General}

In the established context of linear differential inclusions, Proposition 3.1 (2.) provides the connection between solutions to an ODE system for $x(\cdot), X(\cdot)$ and the ellipsoidvalued tube $E:[0, T] \rightsquigarrow \mathbb{R}^{n}, t \mapsto \mathcal{E}(x(t), X(t))$ with three properties. In connection with the more general problem class of set evolution equations, however, we have not made any comment on the last two features so far, i.e.,

3.1 (2.) (c) $E$ is minimal in the class of ellipsoids w.r.t. set inclusions, i.e., for every $t \in[0, T]$, there does not exist any ellipsoid $\widetilde{E} \subset \mathbb{R}^{n}$ with $\mathcal{R}_{A \cdot+B U}\left(t, K_{0}\right) \subset$ $\widetilde{E} \subsetneq E(t)$.

3.1 (2.) (d) For every $t \in[0, T], \mathcal{R}_{A \cdot+B U}\left(t, K_{0}\right)$ and $E(t)$ have a boundary point $\xi(t)$ in common such that $\ell(t)$ is normal to both sets in $\xi(t)$.

The following example shows that such a form of minimality does not hold under the assumptions of Proposition 3.3. In a word, the current set $K(t) \subset \mathbb{R}^{n}$ might have a significant influence on the coefficient matrices $\mathcal{A}(t, K(t)), \mathcal{B}(t, K(t)) \in \mathbb{R}^{n \times n}$ such that joint boundary points are lost instantaneously. 
Example 3.5 For $n=2$, set $A_{0}:=B_{0}:=\mathbb{1}$ (i.e., the unit matrix in $\left.\mathbb{R}^{2 \times 2}\right), U:=\mathcal{E}\left(0, Q_{u}\right)$ and $K_{0}:=\mathcal{E}\left(0, X_{0}\right) \subset \mathbb{R}^{2}$ with $Q_{u}:=\left(\begin{array}{ll}1 & 0 \\ 0 & 2\end{array}\right), X_{0}:=\left(\begin{array}{cc}3 & -1 \\ -1 & 3\end{array}\right) \cdot\left((1,1)^{\top}\right.$ and $(-1,1)^{\top}$ are eigenvectors of $X_{0}$ associated with the eigenvalues 2,4 , respectively.)

The variation of constants formula provides an explicit representation of the reachable set $R(t) \subset \mathbb{R}^{2}$ of the autonomous linear differential inclusion $x^{\prime} \in A_{0} x+B_{0} U$ (see, e.g., [36, Lemma 3.1.1])

$$
\begin{aligned}
R(t) & =\exp \left(t A_{0}\right) \mathcal{E}\left(0, X_{0}\right)+\int_{0}^{t} \exp \left((t-s) A_{0}\right) \cdot B_{0} U \mathrm{~d} s \\
& =e^{t} \cdot \mathcal{E}\left(0, X_{0}\right)+\left(e^{t}-1\right) \cdot \mathcal{E}\left(0, U_{0}\right) .
\end{aligned}
$$

In particular, $R(t) \subset \mathbb{R}^{2}$ is convex, compact, but not an ellipsoid for $t>0$.

In addition to $\mathcal{A}(t, M):=A_{0}=\mathbb{1}$, we define $\mathcal{B}:[0,1] \times \mathcal{K}_{\mathrm{co}}\left(\mathbb{R}^{2}\right) \longrightarrow \mathbb{R}^{2 \times 2}$ in such a way that for all $M \in \mathcal{K}_{\mathrm{co}}\left(\mathbb{R}^{n}\right)$ and $t \in[0,1], \quad R(t) \varsubsetneqq M \Longleftrightarrow \overline{\mathbb{B}}_{1}=$ $\mathcal{B}(t, R(t)) \overline{\mathbb{B}}_{1} \varsubsetneqq \mathcal{B}(t, M) \mathbb{B}_{1}$,

e.g., $\mathcal{B}(t, M):=\left(2-e^{-\mathbb{e}(M, R(t))}\right) \cdot \mathbb{1} \in \mathbb{R}^{2 \times 2}$. Clearly, $R(\cdot)$ is the solution to the IVP (6).

Fixing a unit vector $\ell_{0} \in \mathbb{R}^{2}$ arbitrarily, Proposition 3.3 and Corollary 3.4 provide an ellipsoid-valued tube $E:[0,1] \rightsquigarrow \mathbb{R}^{2}, t \mapsto \mathcal{E}(0, X(t))$ with the following properties:

- $X:[0,1] \longrightarrow \mathbb{R}^{2 \times 2}$ and $\ell:[0,1] \longrightarrow \mathbb{R}^{2}$ solve the ODE system

$$
\begin{aligned}
\ell^{\prime}(t)= & -\ell(t), \\
X^{\prime}(t)= & 2 \quad X(t)+\left(2-e^{-\mathbb{e}(\mathcal{E}(0, X(t)), R(t))}\right) \sqrt{\frac{\left\langle\ell(t), Q_{u} \ell(t)\right\rangle}{\langle\ell(t), X(t) \ell(t)\rangle}} X(t) \\
& +\left(2-e^{-\mathbb{e}(\mathcal{E}(0, X(t)), R(t))}\right) \sqrt{\frac{\langle\ell(t), X(t) \ell(t)\rangle}{\left\langle\ell(t), Q_{u} \ell(t)\right\rangle}} Q_{u}
\end{aligned}
$$

with the initial values $\ell(0)=\ell_{0}$ and $X(0)=X_{0} \stackrel{\text { Def. }}{=}\left(\begin{array}{cc}3 & -1 \\ -1 & 3\end{array}\right)$.

(The equation of the center $x(\cdot)$ in ODE system (7) has the unique solution $x(\cdot)=0$ in this example and so, we do not mention it explicitly any longer.)

- $R(t) \subset E(t)$ holds for every $t \in[0,1]$.

Proposition 3.1 (2.), however, specifies an ellipsoid-valued tube $\widetilde{E}:[0,1] \rightsquigarrow \mathbb{R}^{n}$ with $R(t) \subset \widetilde{E}(t) \subset E(t)^{\circ}$ for all $t \in(0,1]$

Indeed, consider the solution $\widetilde{X}:[0,1] \longrightarrow \mathbb{R}^{2 \times 2}$ of

$\tilde{X}^{\prime}(t)=2 \tilde{X}(t)+\sqrt{\frac{\left\langle\ell(t), Q_{u} \ell(t)\right\rangle}{\langle\ell(t), \tilde{X}(t) \ell(t)\rangle}} \tilde{X}(t)+\sqrt{\frac{\langle\ell(t), \tilde{X}(t) \ell(t)\rangle}{\left\langle\ell(t), Q_{u} \ell(t)\right\rangle}} \quad Q_{u}, \quad \tilde{X}(0)=X_{0}$.

(The adjoint equation for $\ell$ is the same as before: $\ell^{\prime}(t)=-A_{0}^{\top} \ell(t)=-\ell(t)$.) Due to Proposition 3.1 (2.) (c), this $\widetilde{E}(\cdot)$ is minimal w.r.t. set inclusions at every time, i.e., $R(t) \subset \widetilde{E}(t)$ for all $t \in(0,1]$.

Hence, it remains to verify that $\widetilde{E}(t)$ is contained in the interior $E(t)^{\circ}$ for every $t \in(0,1]$. As the reachable set $R(t)$ is not an ellipsoid, we have $R(t) \varsubsetneqq \widetilde{E}(t)$ and so $\mathbb{e}(E(t), R(t)), \mathbb{e}(\widetilde{E}(t), R(t))>0$. First, $\psi:=\langle\ell(\cdot), X(\cdot) \ell(\cdot)\rangle:[0,1] \longrightarrow \mathbb{R}$ is 
LIPSCHITZ continuous with

$$
\begin{aligned}
\frac{\mathrm{d}}{\mathrm{d} s} \psi(s)= & \left\langle\ell^{\prime}(s), X(s) \ell(s)\right\rangle+\left\langle\ell(s), X(s) \ell^{\prime}(s)\right\rangle+\left\langle\ell(s), X^{\prime}(s) \ell(s)\right\rangle \\
& =2 \cdot\left(2-e^{-\mathbb{e}(\mathcal{E}(0, X(s)), R(s))}\right)\left\langle\ell(s), Q_{u} \ell(s)\right\rangle^{\frac{1}{2}} \sqrt{\psi(s)}
\end{aligned}
$$

and thus, $\frac{\mathrm{d}}{\mathrm{d} s} \sqrt{\psi(s)}=\frac{1}{2 \sqrt{\psi(s)}} \cdot \psi^{\prime}(s)=\left(2-e^{-\mathbb{e}(\mathcal{E}(0, X(s)), R(s))}\right) \sqrt{\left\langle\ell(s), Q_{u} \ell(s)\right\rangle}$ for a.e. $s \in[0,1]$. Similarly, $\frac{\mathrm{d}}{\mathrm{d} s} \sqrt{\langle\ell(s), \widetilde{X}(s) \ell(s)\rangle}=\sqrt{\left\langle\ell(s), Q_{u} \ell(s)\right\rangle}$ for a.e. $s \in$ $(0,1]$ and we conclude $\frac{\mathrm{d}}{\mathrm{d} s} \sqrt{\langle\ell(s), \widetilde{X}(s) \ell(s)\rangle}=\eta(s) \cdot \frac{\mathrm{d}}{\mathrm{d} s} \sqrt{\langle\ell(s), X(s) \ell(s)\rangle}$ with some $\eta \in C^{0}([0,1],(0,1])$ satisfying $\eta(s)<1$ whenever $\mathcal{E}(0, X(s)) \neq R(s)$. Due to $\widetilde{X}(0)=X_{0}=X(0)$, it implies $\langle\ell(t), \widetilde{X}(t) \ell(t)\rangle<\langle\ell(t), X(t) \ell(t)\rangle$ for every $t \in(0,1]$.

Second, we consider $\ell^{\perp}(t):=O_{\frac{\pi}{2}} \ell(t) \in \mathbb{R}^{2}$ with the rotation matrix $O_{\frac{\pi}{2}}:=\left(\begin{array}{cc}0 & -1 \\ 1 & 0\end{array}\right)$ in the similar way: ODE system (9) implies for $X^{\perp}(t):=O_{\frac{\pi}{2}}^{\top} X(t) O_{\frac{\pi}{2}}$ at a.e. time instant $s \in[0,1]$

$$
\begin{aligned}
\frac{\mathrm{d}}{\mathrm{d} s} X^{\perp}(s)=2 & X^{\perp}(s)+\left(2-e^{-\mathbb{e}(\mathcal{E}(0, X(s)), R(s))}\right)\left(\sqrt{\frac{\left\langle\ell(s), Q_{u} \ell(s)\right\rangle}{\langle\ell(s), X(s) \ell(s)\rangle}} X^{\perp}(s)\right. \\
& +\sqrt{\frac{\langle\ell(s), X(s) \ell(s)\rangle}{\left\langle\ell(s), Q_{u} \ell(s)\right\rangle}} O_{\frac{\pi}{2}}^{\top} Q_{u} O_{\frac{\pi}{2}}
\end{aligned}
$$

Hence, the same steps as before lead to

$$
\begin{aligned}
& \frac{\mathrm{d}}{\mathrm{d} s} \sqrt{\left\langle\ell^{\perp}(s), \widetilde{X}(s) \ell^{\perp}(s)\right\rangle}=\sqrt{\frac{\left\langle\ell(s), Q_{u} \ell(s)\right\rangle}{\langle\ell(s), X(s) \ell(s)\rangle}}+\sqrt{\frac{\langle\ell(s), X(s) \ell(s)\rangle}{\left\langle\ell(s), Q_{u} \ell(s)\right\rangle} \frac{\left\langle\ell^{\perp}(s), Q_{u} \ell^{\perp}(s)\right\rangle}{\left\langle\ell^{\perp}(s), X(s) \ell^{\perp}(s)\right\rangle}} \\
& \frac{\mathrm{d}}{\mathrm{d} s} \sqrt{\left\langle\ell^{\perp}(s), X(s) \ell^{\perp}(s)\right\rangle}=\left(2-e^{-\mathbb{e}(\mathcal{E}(0, X(s)), R(s))}\right) \cdot \frac{\mathrm{d}}{\mathrm{d}_{s}} \sqrt{\left\langle\ell^{\perp}(s), \widetilde{X}(s) \ell^{\perp}(s)\right\rangle}
\end{aligned}
$$

and thus, $\left\langle\ell^{\perp}(t), \widetilde{X}(t) \ell^{\perp}(t)\right\rangle<\left\langle\ell^{\perp}(t), X(t) \ell^{\perp}(t)\right\rangle$ for every $t \in(0,1]$.

As a consequence, $\widetilde{E}(t) \stackrel{\text { Def. }}{=} \mathcal{E}(0, \widetilde{X}(t))$ is always contained in the interior of $E(t) \stackrel{\text { Def. }}{=}$ $\mathcal{E}(0, X(t)) \subset \mathbb{R}^{2}$. In particular, the reachable tube $R(\cdot)$ is the solution of the underlying IVP (6), but $R(t)$ cannot have a joint boundary point with $E(t)$ for any $t \in(0,1]$.

\subsection{Approximating the Solution of a Set Evolution Equation with Arbitrary Precision}

Example 3.5 shows that the value $K(t)$ of the solution tube might be contained in the interior of any approximating ellipsoid $\mathcal{E}(x(t), X(t))$ based on the ODE system (7), (8). Whenever a joint boundary point (as mentioned in Proposition 3.1 (2.) (d)) does not exist, it is not so obvious how to estimate the gap of the approximation. The following results states that the exact solution can be approximated with arbitrary precision-by choosing the number of ellipsoids sufficiently large. 
Proposition 3.6 Suppose the assumptions of Corollary 3.4 for $\mathcal{A}, \mathcal{B}:[0, T] \times$ $\mathcal{K}_{c o}\left(\mathbb{R}^{n}\right) \longrightarrow \mathbb{R}^{n \times n}$ and $U=\mathcal{E}\left(q_{u}, Q_{u}\right) \subset \mathbb{R}^{n}$. Choose any initial set $K_{0}=$ $\mathcal{E}\left(x_{0}, X_{0}\right) \subset \mathbb{R}^{n}$.

For every $\varepsilon>0$, there exist $N \in \mathbb{N}$ and unit vectors $\ell_{0 j} \in \mathbb{R}^{n}(j=1, \ldots, N)$ such that the following statement holds: Let $\ell_{j}(\cdot), x_{j}(\cdot), X_{j}(\cdot)(j=1, \ldots, N)$ be the unique solutions to ODE system (7) with the initial values $\ell_{j}(0)=\ell_{0 j}, x_{j}(0)=x_{0}$, $X_{j}(0)=X_{0}$. Then, the solution tube $K:[0, T] \rightsquigarrow \mathbb{R}^{n}$ to the IVP (6) satisfies $\mathbb{d}\left(K(t), \mathcal{E}_{\cap}(t)\right)<\varepsilon$ for all $t \in[0, T]$.

\section{A Numerical Example}

This example is deliberately short and in two dimensions so that numerical results can be shown in figures. Motivated by challenges of collision avoidance, we consider a simple cart under the influence of bounded "unknown noise" and aim at a guaranteed state estimation of its position and velocity. In addition, security reasons require a safety zone which grows with the "uncertainty" of the state estimation.

Now we suggest a simple model for this situation. Initially (i.e., without any noise or safety zone), the cart moves according to the linear control system for scalar position $x_{1}$ and velocity $x_{2}=x_{1}^{\prime}$ in which $u_{2}$ describes its acceleration: $x^{\prime}=A x+\left(\begin{array}{c}0 \\ u_{2}\end{array}\right)$ with $A:=\left(\begin{array}{ll}0 & 1 \\ 0 & 0\end{array}\right)$.

Three aspects are implemented additionally: First, initial position and velocity are imprecise and, the error in position grows with the velocity. Here, we choose the initial set $\mathcal{E}\left(x_{0}, X_{0}\right) \subset \mathbb{R}^{2}$ with $x_{0}:=(0,1)^{\top}, X_{0}:=\left(\begin{array}{ll}1 & 0.5 \\ 0.5 & 1\end{array}\right)$ having the eigenvector $(1,1)^{\top}$. Second, there is a scalar "noise" $u_{1}$ whose order of magnitude is small in comparison with the acceleration $u_{2}$. It affects both position $x_{1}$ and velocity $x_{2}$. We choose $U:=\mathcal{E}\left(0, Q_{u}\right) \subset \mathbb{R}^{2}, Q_{u}:=\left(\begin{array}{ll}10^{-3} & 0 \\ 0 & 1\end{array}\right)$ and $B_{0}:=\left(\begin{array}{ll}1 & 0 \\ 1 & 1\end{array}\right)$. The reachable set of $x^{\prime} \in A x+B_{0} U$ consists of all states which the cart can attain-no matter how the acceleration $u_{2}$ and the "noise" $u_{1}$ have evolved. Its set diameter exemplifies a real quantity describing the uncertainty/imprecision of the system-whenever we do not have any influence on $u_{1}, u_{2}$.

The third aspect is due to the safety zone. It is induced by an additional noise term representing an ellipsoidal neighborhood of 0 which depends on the set diameter. We set $\mathcal{B}(t, M):=B_{0}+\varphi(\operatorname{diam} M) \cdot\left(\begin{array}{ll}1 & 0 \\ 1 & 0.5\end{array}\right)$ with the smooth cut-off function $\varphi(s):=\frac{s}{1+s}$ $(s \geq 0)$ and consider $\stackrel{\circ}{K}(t)=A x+\mathcal{B}(t, K(t)) U$.

On the basis of Proposition 3.6, the solution values $K(1), K(10) \subset \mathbb{R}^{2}$ are approximated by the intersections of 400 ellipses with the joint initial set $\mathcal{E}\left(x_{0}, X_{0}\right) \subset \mathbb{R}^{2}$ and the respective direction vector $\ell_{j}(0) \in \mathbb{R}^{2}$ distributed equidistantly in the unit circle. The table shows the numerical values of POMPEIU-HAUSDORFF distance $\mathbb{d}$ between $K(10)$ and the intersection $\mathcal{E}_{\cap, N}(10) \stackrel{\text { Def. }}{=} \bigcap_{k=1}^{N} \mathcal{E}\left(x_{k}(10), X_{k}(10)\right)$ and the gap of 
Table 1 Numerical results for the example in Sect. 4

\begin{tabular}{lll}
\hline $\mathrm{N}$ & $\mathrm{d}\left(\mathcal{E}_{\cap, N}(10), K(10)\right)$ & $\mathbb{e}\left(\partial \mathcal{E}_{\cap, N}(10), K(10)\right)$ \\
\hline 1 & 160.229 & 0.887777 \\
2 & 0.013715 \\
3 & & 0.031389 \\
\hline
\end{tabular}

$\partial \mathcal{E}_{\cap, N}(10)$ and $K(10)$ for various $N$. It is worth mentioning that in this (very simple) example, all these gaps are positive, i.e., $K(10)$ does not have any joint boundary point with $\mathcal{E}_{\cap}(10)$.

\section{Proofs}

\subsection{Tools about Reachable Sets of Differential Inclusions}

Proposition 2.1 specifies sufficient conditions on $U \subset \mathbb{R}^{m}$ and $f:[0, T] \times \mathbb{R}^{n} \times$ $\mathcal{K}\left(\mathbb{R}^{n}\right) \times U \longrightarrow \mathbb{R}^{n}$ such that the three types of set evolution equations are equivalent to each other. Here, we formulate just the key properties of reachable sets relevant for proving the statements in Sects. 2.2 and 2.3. Essentially the same arguments as in the proof of [41, Lemma 1.58] lead to the following statement underlying the equivalence 2.1 " (2.) $\Longleftrightarrow(3)$.$" , for example.$

Lemma 5.1 Let $T>0, U \subset \mathbb{R}^{m}$ be nonempty compact and $g:[0, T] \times \mathbb{R}^{n} \times U \longrightarrow$ $\mathbb{R}^{n}$ satisfy these conditions:

(i) For all $t \in[0, T]$ and $x \in \mathbb{R}^{n}$, the set $g(t, x, U) \stackrel{\text { Def. }}{=}\{g(t, x, u) \mid u \in U\} \subset \mathbb{R}^{n}$ is closed and convex.

(ii) For all $x \in \mathbb{R}^{n}$ and $u \in U, g(\cdot, x, u):[0, T] \longrightarrow \mathbb{R}^{n}$ is LEBESGUE measurable. 
(iii) For all $x \in \mathbb{R}^{n}$ and a.e. $t \in[0, T], g(t, x, \cdot): U \longrightarrow \mathbb{R}^{n}$ is continuous.

(iv) There exists $\lambda \in L^{1}([0, T])$ such that for all $u \in U$ and a.e. $t \in[0, T]$, the function $g(t, \cdot, u): \mathbb{R}^{n} \longrightarrow \mathbb{R}^{n}$ is $\lambda(t)$-LIPSCHITZ continuous.

(v) There is $\Gamma \geq 0$ with $\|g(t, x, u)\| \leq \Gamma(1+\|x\|)$ for all $t \in[0, T], x \in \mathbb{R}^{n}$ and $u \in U$.

Then, there exists a measurable set $\widetilde{J} \subset[0, T]$ of full measure, i.e., $\mathcal{L}^{1}([0, T] \backslash \widetilde{J})=$ 0 , such that the following statements hold for every $t \in \widetilde{J}$ :

(1.) $\lim _{h \downarrow 0} \frac{1}{h} \cdot \mathbb{d}\left(\mathcal{R}_{g(t+\cdot, \cdot, U)}\left(h, M_{0}\right), \bigcup_{x \in M_{0}}(x+h \cdot g(t, x, U))\right)=0$ for every initial set $M_{0} \in \mathcal{K}\left(\mathbb{R}^{n}\right)$.

(2.) For every initial set $M_{0} \in \mathcal{K}\left(\mathbb{R}^{n}\right)$, the POMPEIU-HAUSDORFF distance between the reachable sets of the autonomous inclusion $y^{\prime} \in g(t, y, U)$ and the nonautonomous inclusion $y^{\prime} \in g(t+\cdot, y, U)$ satisfies

$\lim _{h \downarrow 0} \frac{1}{h} \cdot \mathbb{d}\left(\mathcal{R}_{g(t, \cdot, U)}\left(h, M_{0}\right), \mathcal{R}_{g(t+\cdot, \cdot, U)}\left(h, M_{0}\right)\right)=0$.

Lemma 5.2 Let $U \subset \mathbb{R}^{m}$ and $g:[0, T] \times \mathbb{R}^{n} \times U \longrightarrow \mathbb{R}^{n}$ satisfy the assumptions of Lemma 5.1. Consider $K_{0} \in \mathcal{K}\left(\mathbb{R}^{n}\right)$ with $K_{0} \subset \overline{\mathbb{B}}_{r}$. Then,

(1.) For every $t \in[0, T], \mathcal{R}_{g(\cdot, \cdot, U)}\left(t, K_{0}\right)$ is contained in $\overline{\mathbb{B}}_{R(t)} \subset \mathbb{R}^{n}$ with $R(t):=(r+$ $\Gamma t) \cdot e^{\Gamma t}$.

(2.) $\mathcal{R}_{g(\cdot, \cdot, U)}\left(\cdot, K_{0}\right):[0, T] \rightsquigarrow \mathbb{R}^{n}$ is LIPSCHITZ continuous w.r.t. $\mathbb{d}$ and, its LIPSCHITZ constant is $\leq \Gamma(1+r+\Gamma T) \cdot e^{\Gamma T}$.

As a consequence of well-known FILIPPOV's theorem about solutions to differential inclusions, the following bound holds for the POMPEIU-HAUSDORFF distance between reachable sets (see, e.g., the proofs of [4, Proposition 3.7.3], [17, Lemma 5.1] or [41, Propositions 1.50, 2.79]).

Lemma 5.3 (Reachable sets: Continuous dependence on data) Suppose the assumptions of Lemma 5.1 for $U \subset \mathbb{R}^{m}$ and $g_{1}, g_{2}:[0, T] \times \mathbb{R}^{n} \times U \longrightarrow \mathbb{R}^{n}$ (with the same $\lambda \in L^{1}([0, T])$ and $\left.\Gamma \geq 0\right)$.

For all initial sets $\bar{K}_{1}, K_{2} \in \mathcal{K}\left(\mathbb{R}^{n}\right)$ with $K_{1} \cup K_{2} \subset \overline{\mathbb{B}}_{r}$, the following estimate with $R:=(r+\Gamma T) \cdot e^{\Gamma T}$ holds at each time $t \in[0, T]$

$$
\begin{gathered}
\mathbb{d}\left(\mathcal{R}_{g_{1}(\cdot, \cdot, U)}\left(t, K_{1}\right), \mathcal{R}_{g_{2}(\cdot, \cdot, U)}\left(t, K_{2}\right)\right) \\
\leq \mathbb{d}\left(K_{1}, K_{2}\right) \cdot e^{\int_{0}^{t} \lambda(s) \mathrm{d} s}+\int_{0}^{t} \sup _{x \in \overline{\mathbb{B}}_{R}} \mathbb{d}\left(g_{1}(s, x, U), g_{2}(s, x, U)\right) \cdot e^{\int_{s}^{t} \lambda(\tau) \mathrm{d} \tau} \mathrm{d} s .
\end{gathered}
$$

\subsection{Continuous Dependence of Solution Tubes on Data (Proposition 2.5)}

Proof of Proposition 2.5 Consider $\delta:=\mathbb{d}(K(\cdot), M(\cdot)):[0, T] \longrightarrow[0, \infty)$. 
Due to Lemma $5.2(2),. \delta(\cdot)$ is LIPSCHITZ continuous with $\delta(0)=\mathbb{d}(K(0), M(0))$. We conclude from the criterion 2.1 (3.) and Lemma 5.3 that for a.e. $t \in[0, T)$

$$
\begin{aligned}
& \underset{h \downarrow 0}{\limsup \operatorname{su}} \frac{\delta(t+h)-\delta(t)}{h} \\
& \leq \underset{h \downarrow 0}{\limsup } \frac{1}{h} \cdot \mathbb{d}\left(K(t+h), \mathcal{R}_{f_{1}(t, \cdot, K(t), U)}(h, K(t))\right) \\
& +\underset{h \downarrow 0}{\limsup } \frac{1}{h} \cdot \mathbb{d}\left(\mathcal{R}_{f_{1}(t, \cdot, K(t), U)}(h, \quad K(t)), \mathcal{R}_{f_{1}(t, \cdot, M(t), U)}(h, \quad K(t))\right) \\
& +\underset{h \downarrow 0}{\limsup } \frac{1}{h}\left(\mathbb{d}\left(\mathcal{R}_{f_{1}(t, \cdot, M(t), U)}(h, \quad K(t)), \mathcal{R}_{f_{2}(t, \cdot, M(t), U)}(h, M(t))\right)-\delta(t)\right) \\
& +\limsup _{h \downarrow 0} \frac{1}{h} \cdot \mathbb{d}\left(\mathcal{R}_{f_{2}(t, \cdot, M(t), U)}(h, \quad M(t)), M(t+h)\right) \\
& \quad \limsup _{h \downarrow 0} \frac{\delta(t+h)-\delta(t)}{h} \leq 0+\sup _{x \in \overline{\mathbb{B}}_{R}} \mathbb{d}\left(f_{1}(t, x, K(t), U), f_{1}(t, x, M(t), U)\right) \\
& \quad+\lambda \delta(t)+\sup _{x \in \overline{\mathbb{B}}_{R}} \mathbb{d}\left(f_{1}(t, x, M(t), U), f_{2}(t, x, M(t), U)\right)+0 .
\end{aligned}
$$

By Assumption $2.2\left(\mathrm{v}^{\prime}\right), f_{1}(t, x, \cdot, u):\left(\mathcal{K}\left(\mathbb{R}^{n}\right), \mathbb{d}\right) \longrightarrow \mathbb{R}^{n}$ is $\Lambda(t)$-LIPSCHITZ for all $x, u$ and a.e. $t$. Hence,

$$
\begin{aligned}
& \limsup _{h \downarrow 0} \frac{\delta(t+h)-\delta(t)}{h} \\
& \leq(\Lambda(t)+\lambda) \cdot \delta(t)+\sup _{x \in \overline{\mathbb{B}}_{R}} \mathbb{d}\left(f_{1}(t, x, M(t), U), f_{2}(t, x, M(t), U)\right)
\end{aligned}
$$

for a.e. $t \in[0, T]$ and, GRONWALL's inequality leads to the claimed estimate.

\subsection{Inclusion Principle of Solution Tubes (Proposition 2.7)}

The gist of the proof is to reformulate the condition $K \subset M_{1} \cap M_{2}$ as a constraint on tuples $\left(K, M_{1}, M_{2}\right) \in \mathcal{K}\left(\mathbb{R}^{n}\right)^{3} \cdot \mathcal{C}:=\left\{\left(K, M_{1}, M_{2}\right) \in \mathcal{K}\left(\mathbb{R}^{n}\right)^{3} \mid K \subset M_{1} \cap M_{2}\right\}$ proves to be invariant w.r.t. the system

$\stackrel{\circ}{K}(t)=f(t, \cdot, K(t), U), \quad \stackrel{\circ}{M}(t)=g(t, \cdot, M(t), U), \quad \stackrel{\stackrel{M}{M}}{(t)}=\widetilde{g}(t, \cdot, \tilde{M}(t), U)$.

Weak invariance (a.k.a. viability) has already been investigated by AUBIN and GORRE (e.g., [4, § 4.3.3], [26,27]). Now we use some of their technical results for verifying the (strong) invariance of $\mathcal{C}$.

Lemma $5.4\left[4\right.$, Lemma 4.2.7] Supply the product $\mathcal{K}\left(\mathbb{R}^{n}\right)^{3}$ with the metric

$$
\begin{aligned}
& \mathbb{d}_{3}: \mathcal{K}\left(\mathbb{R}^{n}\right)^{3} \times \mathcal{K}\left(\mathbb{R}^{n}\right)^{3} \longrightarrow \mathbb{R}, \quad\left(\left(K_{1}, K_{2}, K_{3}\right),\left(M_{1}, M_{2}, M_{3}\right)\right) \\
& \quad \longmapsto \mathbb{d}\left(K_{1}, M_{1}\right)+\mathbb{d}\left(K_{2}, M_{2}\right)+\mathbb{d}\left(K_{3}, M_{3}\right) .
\end{aligned}
$$


Then, $\mathcal{C} \stackrel{\text { Def. }}{=}\left\{\left(K, M_{1}, M_{2}\right) \in \mathcal{K}\left(\mathbb{R}^{n}\right)^{3} \mid K \subset M_{1} \cap M_{2}\right\}$ is closed in $\left(\mathcal{K}\left(\mathbb{R}^{n}\right)^{3}, \mathbb{d}_{3}\right)$.

Lemma 5.5 (GORRE [4, Theorem 4.2.8] [26]) Let $U \subset \mathbb{R}^{m}$ be nonempty compact and $\widetilde{g}_{1}, \widetilde{g}_{2}, \widetilde{g}_{3}: \mathbb{R}^{n} \times U \longrightarrow \mathbb{R}^{n}$ satisfy the following conditions:

(i) For all $x \in \mathbb{R}^{n}$, the set $\widetilde{g}_{j}(x, U) \subset \mathbb{R}^{n}$ is compact and convex.

(ii) For every $x \in \mathbb{R}^{n}, \widetilde{g}_{j}(x, \cdot): U \longrightarrow \mathbb{R}^{n}$ is continuous.

(iii) There exists $\lambda>0$ such that for each $u \in U, \widetilde{g}_{j}(\cdot, u): \mathbb{R}^{n} \longrightarrow \mathbb{R}^{n}$ is $\lambda$ LIPSCHITZ continuous.

Suppose that $\left(K, M_{1}, M_{2}\right) \in \mathcal{C}$ fulfills $\widetilde{g}_{1}(x, U) \subset\left(\widetilde{g}_{2}(x, U)+T_{M_{1}}^{b}(x)\right) \cap\left(\widetilde{g}_{3}(x, U)+\right.$ $\left.T_{M_{2}}^{b}(x)\right)$ for all $x \in K$.

Then, the tuple $\left(\widetilde{g}_{1}(\cdot, U), \widetilde{g}_{2}(\cdot, U), \widetilde{g}_{3}(\cdot, U)\right)$ of LIPSCHITZ maps $\mathbb{R}^{n} \rightsquigarrow \mathbb{R}^{n}$ is contingent to $\mathcal{C} \subset \mathcal{K}\left(\mathbb{R}^{n}\right)^{3}$ at $\left(K, M_{1}, M_{2}\right)$ in the sense of [4, Definition 1.5.2], i.e., the following equivalent conditions hold:

(1.) $\liminf _{h \downarrow 0} \frac{1}{h} \cdot \operatorname{dist}_{\mathbb{d}_{3}}\left(\left(\mathcal{R}_{\widetilde{g}_{1}(\cdot, U)}(h, K), \mathcal{R}_{\widetilde{g}_{2}(\cdot, U)}\left(h, M_{1}\right), \mathcal{R}_{\widetilde{g}_{3}(\cdot, U)}\left(h, M_{2}\right)\right), \mathcal{C}\right)=$ 0 .

(2.) There are sequences $\left(h_{\ell}\right)_{\ell \in \mathbb{N}}$ and $\left(\left(K_{\ell}, M_{1, \ell}, M_{2, \ell}\right)\right)_{\ell \in \mathbb{N}}$ in $\mathbb{R}$ and $\mathcal{K}\left(\mathbb{R}^{n}\right)^{3}$, respectively, such that for every $\ell \in \mathbb{N}$,

$$
\begin{array}{ll}
0<h_{\ell}<\frac{1}{\ell}, K_{\ell} \subset M_{1, \ell} \cap M_{2, \ell}, & \mathbb{d}\left(\mathcal{R}_{\widetilde{g}_{1}(\cdot, U)}\left(h_{\ell}, \quad K\right), K_{\ell}\right) \leq \frac{1}{\ell} h_{\ell}, \\
\mathbb{d}\left(\mathcal{R}_{\widetilde{g}_{2}(\cdot, U)}\left(h_{\ell}, M_{1}\right), M_{1, \ell}\right) \leq \frac{1}{\ell} h_{\ell}, & \mathbb{d}\left(\mathcal{R}_{\widetilde{g}_{3}(\cdot, U)}\left(h_{\ell}, \quad M_{2}\right), M_{2, \ell}\right) \leq \frac{1}{\ell} h_{\ell} .
\end{array}
$$

The next lemma extends (forward) LEBESGUE points to measurable functions with values in a metric space $Y$.

Lemma 5.6 Let $Y$ be a metric space. Suppose for $\psi:[0, T] \longrightarrow Y$ and $\Delta: Y \times Y \longrightarrow$ $[0, \infty):$

(i) $\psi(\cdot)$ is LEBESGUE measurable, $\Delta(\cdot)$ is continuous and satisfies the triangle inequality.

(ii) For some $y_{0} \in Y, M:=\max \left\{\Delta\left(y_{0}, \psi(\cdot)\right), \Delta\left(\psi(\cdot), y_{0}\right)\right\}:[0, T] \longrightarrow \mathbb{R}$ is integrable.

Then, $\lim _{h \downarrow 0} \frac{1}{h} \int_{t}^{t+h} \Delta(\psi(t), \psi(s)) \mathrm{d} s=0$ holds for a.e. $t \in[0, T)$.

Proof Choose any sequence $\left(\varepsilon_{\ell}\right)_{\ell \in \mathbb{N}}$ in $(0,1)$ with $\sum_{\ell=1}^{\infty} \varepsilon_{\ell}<\infty$. For each $\ell \in \mathbb{N}$, LUSIN's Theorem for metric-valued functions provides a compact subset $I_{\ell} \subset[0, T]$ with $\mathcal{L}^{1}\left([0, T] \backslash I_{\ell}\right)<\varepsilon_{\ell}$ such that $\left.\psi\right|_{I_{\ell}}: I_{\ell} \longrightarrow Y$ is continuous (e.g., [11, Theorem 7.14.25] citing [24,32]). Set $\widetilde{J}_{k}:=\bigcap_{\ell \geq k} I_{\ell} \subset \mathbb{R}$ for $k \in \mathbb{N}$. Let $\chi_{[0, T] \backslash \widetilde{J}_{k}}: \mathbb{R} \longrightarrow$ $\{0,1\}$ denote the characteristic function of $[0, T] \backslash \widetilde{J}_{k} \subset \mathbb{R}(k \in \mathbb{N}) \cdot \chi_{[0, T] \backslash \widetilde{J}_{k}} \cdot M$ is also integrable. Hence, the set $J_{k}$ of all $t \in[0, T)$ with

$$
\begin{aligned}
& \lim _{h \downarrow 0} \frac{1}{h} \int_{t}^{t+h}\left|\chi_{[0, T] \backslash \widetilde{J_{k}}}(t)-\chi_{[0, T] \backslash \widetilde{J_{k}}}(s)\right| \mathrm{d} s=0 \\
& \quad=\lim _{h \downarrow 0} \frac{1}{h} \int_{t}^{t+h}\left|\left(\chi_{[0, T] \backslash \widetilde{J_{k}}} M\right)(t)-\left(\chi_{[0, T] \backslash \widetilde{J_{k}}} M\right)(s)\right| \mathrm{d} s
\end{aligned}
$$


is of full measure (see, e.g., [60, Ch. 3, Corollary 1.6])). We obtain for all $k \in \mathbb{N}$ and $t \in \widetilde{J}_{k} \cap J_{k}$

$$
\begin{aligned}
& \lim _{h \downarrow 0} \frac{1}{h} \mathcal{L}^{1}\left([t, t+h] \backslash \widetilde{J}_{k}\right)=\chi_{[0, T] \backslash \widetilde{J}_{k}}(t)=0 \\
& \lim _{h \downarrow 0} \frac{1}{h} \int_{[t, t+h] \backslash \widetilde{J}_{k}} M(s) \mathrm{d} s=\chi_{[0, T] \backslash \widetilde{J}_{k}}(t) \cdot M(t)=0 \\
& \lim _{h \downarrow 0} \frac{1}{h} \int_{[t, t+h] \cap \widetilde{J}_{k}} \Delta(\psi(t), \psi(s)) \mathrm{d} s=0
\end{aligned}
$$

as the composition $[0, T] \cap \widetilde{J}_{k} \ni s \longmapsto \Delta(\psi(t), \psi(s))$ is continuous. It implies $\lim _{h \downarrow 0} \frac{1}{h} \int_{t}^{t+h} \Delta(\psi(t), \psi(s)) \mathrm{d} s=0$ for all $k \in \mathbb{N}$ and $t \in \widetilde{J}_{k} \cap J_{k}$. Finally, this limit holds for a.e. $t \in[0, T]$ since $\widetilde{J}_{k} \subset \widetilde{J}_{k+1}$ for all $k \in \mathbb{N}$ and $\mathcal{L}^{1}\left([0, T] \backslash \widetilde{J}_{k}\right) \leq$ $\sum_{\ell=k}^{\infty} \mathcal{L}^{1}\left([0, T] \backslash I_{\ell}\right) \leq \sum_{\ell=k}^{\infty} \varepsilon_{\ell} \longrightarrow 0(k \rightarrow \infty)$

Proof of 2.7 We adapt the arguments usually used for (strong) invariance theorems of differential equations or inclusions (see, e.g., [1, § 5.3], [41, Proposition A.8], [64, $\S 10 . \mathrm{XVI}])$.

Consider $\delta:[0, T] \longrightarrow[0, \infty)$ with

$$
\delta(t)=\operatorname{dist}_{\mathbb{d}_{3}}((K(t), M(t), \widetilde{M}(t)), \mathcal{C}) \stackrel{\text { Def. }}{=} \inf _{\left(M_{0}, M_{1}, M_{2}\right) \in \mathcal{C}}\left(\mathbb{d}\left(K(t), M_{0}\right)+\mathbb{d}\left(M(t), M_{1}\right)+\mathbb{d}\left(\widetilde{M}(t), M_{2}\right)\right)
$$

$\delta(\cdot)$ is LIPSCHITZ continuous since so is each of the tubes $K(\cdot), M(\cdot), \widetilde{M}(\cdot)$. Due to $\delta(0)=0$, it remains to verify $\delta^{\prime}(t) \leq(\Lambda+\lambda) \cdot \delta(t)$ for a.e. $t \in[0, T)$ because then GRONWALL's inequality leads to $\delta(t)=0$ for all $t \in[0, T]$.

The single-valued function $[0, T] \times\left(\mathbb{R}^{n} \times \mathcal{K}\left(\mathbb{R}^{n}\right)\right) \longrightarrow \mathcal{K}\left(\mathbb{R}^{n}\right),(t, x, S) \longmapsto$ $f(t, x, S, U)$ is measurable/ LIPSCHITZ-in the following sense:

- For all $(t, x, S) \in[0, T] \times \mathbb{R}^{n} \times \mathcal{K}\left(\mathbb{R}^{n}\right)$, the set $f(t, x, S, U) \stackrel{\text { Def. }}{=}\{f(t, x, S, u) \mid u$ $\in U\}$ is compact due to continuity assumption 2.1 (iii) and the compactness of $U$.

- For every $(x, S) \in \mathbb{R}^{n} \times \mathcal{K}\left(\mathbb{R}^{n}\right), f(\cdot, x, S, U):[0, T] \longrightarrow\left(\mathcal{K}\left(\mathbb{R}^{n}\right)\right.$, d $)$ is measurable.

Indeed, $f(\cdot, x, S, \cdot):[0, T] \times U \longrightarrow \mathbb{R}^{n}$ is a CARATHÉODORY function due to Assumptions 2.1 (ii),(iii). Fix $\varepsilon>0$ arbitrarily. Then, the SCORZA-DRAGONI theorem (e.g., [56, Theorem 1]) provides a closed subset $J_{\varepsilon} \subset[0, T]$ with $\mathcal{L}^{1}\left([0, T] \backslash J_{\varepsilon}\right)<\varepsilon$ such that $\left.f(\cdot, x, S, \cdot)\right|_{J_{\varepsilon} \times U}$ is continuous. This restriction is even uniformly continuous since $J_{\varepsilon} \times U$ is compact. As a consequence, $\left.f(\cdot, x, S, U)\right|_{J_{\varepsilon}}: J_{\varepsilon} \longrightarrow\left(\mathcal{K}\left(\mathbb{R}^{n}\right), \mathbb{d}\right)$ is continuous. We conclude from LusiN's theorem (e.g., [24,32]) that $f(\cdot, x, S, U):[0, T] \longrightarrow\left(\mathcal{K}\left(\mathbb{R}^{n}\right), \mathbb{d}\right)$ is measurable.

- For every $t \in[0, T], f(t, \cdot, \cdot, U): \mathbb{R}^{n} \times \mathcal{K}\left(\mathbb{R}^{n}\right) \longrightarrow \mathcal{K}\left(\mathbb{R}^{n}\right)$ is $(\Lambda+\lambda)$-LIPSCHITZ continuous as a consequence of assumptions 2.1 (iv) and 2.7 (v') (about the partial Lipschitz continuity of $f(t, \cdot, \cdot, u)$ uniform in $t, u)$. 
In particular, for every $x \in \mathbb{R}^{n}, f(\cdot, x, \cdot, U):[0, T] \times \mathcal{K}\left(\mathbb{R}^{n}\right) \longrightarrow\left(\mathcal{K}\left(\mathbb{R}^{n}\right), \mathbb{d}\right)$, $(t, S) \longmapsto f(t, x, S, U)$ is a CARATHÉODORY function. Thus, the composition $[0, T] \longrightarrow \mathcal{K}\left(\mathbb{R}^{n}\right), t \longmapsto f(t, x, K(t), U)$ is measurable. For the same reasons, $[0, T] \longrightarrow \mathcal{K}\left(\mathbb{R}^{n}\right), t \longmapsto g(t, x, M(t), U)$ and $\widetilde{g}(\cdot, x, \widetilde{M}(\cdot), U)$ are also measurable. Fix $R>0$ sufficiently large such that $K(t), M(t), \tilde{M}(t) \subset B_{R}$ for all $t \in[0, T]$. Let $\left\{x_{1}, x_{2}, \ldots\right\}$ be a countable dense subset of $\overline{\mathbb{B}}_{R} \subset \mathbb{R}^{n}$. Define $I_{0}$ as the set of all $t \in[0, T)$ such that

- $\delta(\cdot)$ is differentiable at $t$

- the inclusion condition 2.7 (vii') holds at $t$ and

- for every $k \in \mathbb{N}, \lim _{h \downarrow 0} \frac{1}{h} \cdot \int_{t}^{t+h} \mathbb{d}\left(f\left(t, x_{k}, K(t), U\right), f\left(s, x_{k}, K(s), U\right)\right) \mathrm{d} s=0$ and the same for $g, \widetilde{g}$.

Due to RADEMACHER's theorem and Lemma 5.6, $I_{0} \subset[0, T]$ is of full measure, i.e., $\mathcal{L}^{1}\left([0, T] \backslash I_{0}\right)=0$.

For every $t \in I_{0}$, there is $\left(K_{t}, M_{t}, \tilde{M}_{t}\right) \in \mathcal{C} \subset \mathcal{K}\left(\mathbb{R}^{n}\right)^{3}$ with $\mathbb{d}_{3}((K(t), M(t)$, $\left.\tilde{M}(t)),\left(K_{t}, M_{t}, \tilde{M}_{t}\right)\right)=\delta(t)$. Indeed, first, all closed balls in $\left(\mathcal{K}\left(\mathbb{R}^{n}\right), \mathbb{d}\right)$ are compact due to [7, Theorem 3.2.4] and so it is in $\left(\mathcal{K}\left(\mathbb{R}^{n}\right)^{3}, \mathbb{d}_{3}\right)$ then. Second, $\mathcal{C}$ is closed w.r.t. $\mathbb{d}_{3}$ according to Lemma 5.4.

Due to the inclusion condition 2.7 (vii'), Lemma 5.5 applied to $\widetilde{g}_{1}:=f\left(t, \cdot, K_{t}, \cdot\right)$, $\widetilde{g}_{2}:=g\left(t, \cdot, M_{t}, \cdot\right), \widetilde{g}_{3}:=\widetilde{g}\left(t, \cdot, \widetilde{M}_{t}, \cdot\right): \mathbb{R}^{n} \times U \longrightarrow \mathbb{R}^{n}$ provides a sequence $\left(h_{\ell}\right)_{\ell \in \mathbb{N}}$ in $(0, T-t)$ tending to 0 such that for each $\ell \in \mathbb{N}$,

$\operatorname{dist}_{\mathbb{d}_{3}}\left(\left(\mathcal{R}_{f\left(t, \cdot, K_{t}, U\right)}\left(h_{\ell}, K_{t}\right), \mathcal{R}_{g\left(t, \cdot, M_{t}, U\right)}\left(h_{\ell}, M_{t}\right), \mathcal{R}_{\widetilde{g}_{\left(t, \cdot, \tilde{M}_{t}, U\right)}}\left(h_{\ell}, \tilde{M}_{t}\right)\right), \mathcal{C}\right)<\frac{3}{\ell} h_{\ell}$.

Lemma 5.3 implies for every $\ell \in \mathbb{N}$

$$
\begin{aligned}
& \delta\left(t+h_{\ell}\right) \stackrel{\text { Def. }}{=} \operatorname{dist}_{\mathbb{d}_{3}}\left(\left(K\left(t+h_{\ell}\right), M\left(t+h_{\ell}\right), \widetilde{M}\left(t+h_{\ell}\right)\right), \mathcal{C}\right) \\
& \leq \mathbb{d}\left(\mathcal{R}_{f(t+\cdot, \cdot, K(t+\cdot), U)}\left(h_{\ell}, \quad K(t)\right), \mathcal{R}_{f\left(t, \cdot, K_{t}, U\right)}\left(h_{\ell}, \quad K_{t}\right)\right) \\
& +\mathbb{d}\left(\mathcal{R}_{g(t+\cdot, \cdot, M(t+\cdot), U)}\left(h_{\ell}, \quad M(t)\right), \mathcal{R}_{g\left(t, \cdot, M_{t}, U\right)}\left(h_{\ell}, \quad M_{t}\right)\right) \\
& +\mathbb{d}\left(\mathcal{R}_{\widetilde{g}(t+\cdot, \cdot, \tilde{M}(t+\cdot), U)}\left(h_{\ell}, \quad \tilde{M}(t)\right), \mathcal{R}_{\widetilde{g}\left(t, r, \widetilde{M}_{t}, U\right)}\left(h_{\ell}, \quad \tilde{M}_{t}\right)\right)+\frac{3}{\ell} h_{\ell} \\
& \leq \mathbb{d}_{3}\left((K(t), M(t), \tilde{M}(t)),\left(K_{t}, M_{t}, \tilde{M}_{t}\right)\right) \cdot e^{\lambda} h_{\ell}+\frac{3}{\ell} h_{\ell} \\
& +\int_{0}^{h_{\ell}}\left(\sup _{x \in \bar{B}_{R}} \mathbb{d}(f(t+\sigma, x, \quad K(t+\sigma), U), \quad f(t, x, K(t), U))+\Lambda \cdot \mathbb{d}\left(K(t), K_{t}\right)\right. \\
& +\sup _{x \in \overline{\mathbb{B}}_{R}} \mathbb{d}(g(t+\sigma, x, \quad M(t+\sigma), U), \quad g(t, x, \quad M(t), U))+\Lambda \cdot \mathbb{d}\left(M(t), M_{t}\right) \\
& \left.+\sup _{x \in \overline{\mathbb{B}}_{R}} \mathbb{d}(\widetilde{g}(t+\sigma, x, \quad \tilde{M}(t+\sigma), U), \quad \tilde{g}(t, x, \quad \tilde{M}(t), U))+\Lambda \cdot \mathbb{d}\left(\widetilde{M}(t), \widetilde{M}_{t}\right)\right) \mathrm{d} \sigma \cdot e^{\lambda h_{\ell}} \\
& \leq \delta(t) \cdot e^{\lambda h_{\ell}}+\frac{3}{\ell} h_{\ell}+h_{\ell} \cdot \Lambda \delta(t) \cdot e^{\lambda h_{\ell}} \\
& +\int_{t}^{t+h_{\ell}}\left(\sup _{x \in \mathbb{B}_{R}} \mathbb{d}(f(s, x, \quad K(s), U),\right.
\end{aligned}
$$




$$
\begin{aligned}
& f(t, x, K(t), U))+\sup _{x \in \overline{\mathbb{B}}_{R}} \mathbb{d}(g(s, x, M(s), U), g(t, x, M(t), U)) \\
+ & \left.\sup _{x \in \mathbb{B}_{R}} \mathbb{d}(\tilde{g}(s, x, \quad \tilde{M}(s), U), \quad \tilde{g}(t, x, \quad \tilde{M}(t), U))\right) \mathrm{d} s \cdot e^{\lambda h_{\ell}} .
\end{aligned}
$$

Fix any $\varepsilon>0$. There exist finitely many $x_{\left(k_{1}\right)}, x_{\left(k_{2}\right)}, \ldots, x_{\left(k_{N}\right)} \in \overline{\mathbb{B}}_{R}$ with $\overline{\mathbb{B}}_{R} \subset$ $\bigcup_{v=1}^{N} \mathbb{B} \frac{\varepsilon}{6(1+\lambda)}\left(x_{\left(k_{v}\right)}\right)$. LIPSCHITZ assumption 2.1 (iv) (w.r.t. $x$ ) leads to

$$
\begin{aligned}
& \delta\left(t+h_{\ell}\right) \leq \delta(t) \cdot e^{(\Lambda+\lambda) h_{\ell}}+\frac{3}{\ell} h_{\ell} \\
& \quad+\int_{t}^{t+h_{\ell}}\left(\sup _{\nu \leq N} \mathbb{d}\left(f\left(s, x_{\left(k_{v}\right)}, \quad K(s), U\right), \quad f\left(t, x_{\left(k_{v}\right)}, \quad K(t), U\right)\right)+2 \lambda \cdot \frac{\varepsilon}{6(1+\lambda)}\right. \\
& \quad+\sup _{\nu \leq N} \mathbb{d}\left(g\left(s, x_{\left(k_{v}\right)}, \quad M(s), U\right), \quad g\left(t, x_{\left(k_{v}\right)}, M(t), U\right)\right)+2 \lambda \cdot \frac{\varepsilon}{6(1+\lambda)} \\
& +\sup _{\nu \leq N} \mathbb{d}\left(\tilde{g}\left(s, x_{\left(k_{v}\right)}, \quad \tilde{M}(s), U\right),\right. \\
& \left.\left.\quad \tilde{g}\left(t, x_{\left(k_{v}\right)}, \quad \tilde{M}(t), U\right)\right)+2 \lambda \cdot \frac{\varepsilon}{6(1+\lambda)}\right) \mathrm{d} s \cdot e^{\lambda h_{\ell} .}
\end{aligned}
$$

Finally, we obtain $\delta^{\prime}(t)=\lim _{h \rightarrow 0} \frac{\delta(t+h)-\delta(t)}{h}=\lim _{\ell \rightarrow \infty} \frac{\delta\left(t+h_{\ell}\right)-\delta(t)}{h_{\ell}} \leq(\Lambda+\lambda)$. $\delta(t)+\varepsilon$ for every $t \in I_{0}$ (with $\varepsilon>0$ fixed arbitrarily small and independently of $t)$.

\subsection{External Ellipsoidal Approximations of Solution Tubes (Proposition 3.2)}

Lemma 5.7 (Intersecting two convex sets $[47, \S \S 5-8]$ )

(1.) Let $M$ and $N$ denote two convex subsets of a normed space E. If $N$ contains an open ball $\mathbb{B}_{\rho}\left(x_{0}\right) \subset E$ with $x_{0} \in M$ and $\rho>0$, then the following inequality holds for every $x \in E$

$$
\operatorname{dist}(x, M \cap N) \leq\left(1+\frac{1}{\rho}\left\|x-x_{0}\right\|\right) \cdot(\operatorname{dist}(x, M)+\operatorname{dist}(x, N)) .
$$

(2.) Let $\mathcal{T}$ be a topological space, $E$ a normed space, $t_{0} \in \mathcal{T}$ and $M, N: \mathcal{T} \rightsquigarrow E$ set-valued maps with convex values. Suppose $M$ and $N$ are continuous (w.r.t. d) at $t_{0}$ and $M\left(t_{0}\right) \cap N\left(t_{0}\right)^{\circ} \subset E$ is nonempty and bounded. Then, $\mathcal{T} \rightsquigarrow E$, $t \mapsto M(t) \cap N(t)$ is continuous (w.r.t. d) at $t_{0}$.

(3.) Let $E$ be a normed space and $M, N:[0, T] \rightsquigarrow E$ convex-valued tubes. Assume for each $t \in[0, T]$ that $M(t) \cap N(t)$ is bounded and the intersection of $M(t)$ and the interior of $N(t)$ is nonempty. If both $M(\cdot)$ and $N(\cdot)$ are LIPSCHITZ continuous, so is also $M(\cdot) \cap N(\cdot)$.

Proof of Proposition 3.2 Set $\mathcal{E}_{\cap}(t):=\bigcap_{j=1}^{N} E_{j}(t)$ for $t \in[0, T]$. We consider the auxiliary function

$$
\delta:[0, T] \longrightarrow[0, \infty], \quad t \longmapsto\left\{\begin{array}{lll}
\mathbb{e}\left(K(t), \mathcal{E}_{\cap}(t)\right) & \text { if } & \mathcal{E}_{\cap}(t) \neq \emptyset \\
\infty & \text { if } & \mathcal{E}_{\cap}(t)=\emptyset
\end{array}\right.
$$


and aim at verifying $\delta(t)=0$ for all $t \in[0, T] . \delta(0)=0$ holds due to the assumption $K(0)=K_{0} \subset \mathcal{E}_{\cap}(0)$.

At every time instant $t \in[0, T]$, the convex set $K(t) \subset \mathbb{R}^{n}$ coincides with the reachable set of $K_{0}$ and the nonautonomous linear differential inclusion $x^{\prime} \in \mathcal{A}(s, K(s)) x+$ $\mathcal{B}(s, K(s)) U$ (a.e.), which can be represented by means of the variation of constants formula (see, e.g., [33, $\S 1.1,1.2]$ ). As a consequence, each $K(t) \subset \mathbb{R}^{n}$ has in common with $K(0)=K_{0}$ that its interior is not empty. Furthermore, $K(\cdot)$ is LIPSCHITZ continuous. Hence, there exists a radius $r_{0}>0$ such that for every $t \in[0, T], K(t)$ contains a closed ball with radius $2 r_{0}$. Choose $\Delta>0$ sufficiently small such that its product with the maximum of the LIPSCHITZ constants of $K(\cdot), E_{1}(\cdot), \ldots, E_{N}(\cdot)$ is bounded by $r_{0}$.

Now consider any $t_{0} \in[0, T]$ with $\delta\left(t_{0}\right)=0$, i.e., $K\left(t_{0}\right) \subset \mathcal{E}_{\cap}\left(t_{0}\right)$. Then, there exists $x_{0} \in \mathbb{R}^{n}$ such that $\overline{\mathbb{B}}_{2 r_{0}}\left(x_{0}\right) \subset K\left(t_{0}\right) \subset \mathcal{E}_{\cap}\left(t_{0}\right)$. Set $\widetilde{T}:=\min \left\{t_{0}+\Delta, T\right\} \in\left(t_{0}, T\right]$ and we obtain for all $s \in\left[t_{0}, \widetilde{T}\right]$

$$
\max _{k=1, \ldots, N} \mathbb{d}\left(E_{k}\left(t_{0}\right), E_{k}(s)\right) \leq r_{0} \Longrightarrow \mathbb{B}_{r_{0}}\left(x_{0}\right) \subset \mathcal{E}_{\cap}(s)
$$

From now on, we focus on the restriction of $\delta$ to $\left[t_{0}, \widetilde{T}\right]$.

$\delta$ is LIPSCHITZ continuous in $\left[t_{0}, \widetilde{T}\right]$ as a consequence of Lemma 5.7 (3.).

Choose $\varepsilon \in\left(0, \widetilde{T}-t_{0}\right)$ arbitrarily. According to the SCORZA-DRAGONI theorem, there exists a closed subset $\widetilde{J}_{\varepsilon} \subset\left[t_{0}, \widetilde{T}\right]$ with $\mathcal{L}^{1}\left(\left[t_{0}, \widetilde{T}\right] \backslash \widetilde{J}_{\varepsilon}\right)<\frac{\varepsilon}{4}$ such that both $\left.\mathcal{A}\right|_{\widetilde{J}_{\varepsilon} \times \mathcal{K}_{\mathrm{co}}\left(\mathbb{R}^{n}\right)}$ and $\left.\mathcal{B}\right|_{\widetilde{J}_{\varepsilon} \times \mathcal{K}_{\mathrm{co}}\left(\mathbb{R}^{n}\right)}$ are continuous.

Then, there is a closed subset $J_{\varepsilon}$ of $\left[t_{0}, \widetilde{T}\right.$ ) with the following properties:

- $J_{\varepsilon} \subset \widetilde{J}_{\varepsilon}$ and $\mathcal{L}^{1}\left(\left[t_{0}, \tilde{T}\right] \backslash J_{\varepsilon}\right)<\frac{\varepsilon}{2}$.

- At every time instant $\tau \in J_{\varepsilon}$, each $E_{j}(\cdot)(j=1, \ldots, N)$ satisfies condition 3.2 (vii).

- Each $\tau \in J_{\varepsilon}$ is a (forward) LEBESGUE point of the characteristic function $\chi_{\widetilde{J}_{\varepsilon}}$ : $[0, T] \longrightarrow\{0,1\}$ of $\widetilde{J}_{\varepsilon}$, i.e., $\quad \lim _{\substack{h \downarrow 0 \\(\tau+h \in[0, T])}} \frac{1}{h} \cdot \mathcal{L}^{1}\left([\tau, \tau+h] \cap \widetilde{J}_{\varepsilon}\right)=\chi \widetilde{J}_{\varepsilon}(\tau)=1$.

Choose any $\tau \in J_{\varepsilon}$. Then, the excess condition 3.2 (vii) also holds for $\mathcal{E}_{\cap}(\tau)$, i.e.,

$$
\lim _{h \downarrow 0} \frac{1}{h} \cdot \mathbb{e}\left(\left(\mathbb{1}+h \mathcal{A}\left(\tau, \mathcal{E}_{\cap}(\tau)\right)\right) \mathcal{E}_{\cap}(\tau)+h \mathcal{B}\left(\tau, \mathcal{E}_{\cap}(\tau)\right) U, \quad \mathcal{E}_{\cap}(\tau+h)\right)=0
$$

Indeed, Lemma 5.7 (1.) implies for all $h \in(0, \widetilde{T}-\tau), x \in \mathcal{E}_{\cap}(\tau)$ and $u \in U$

$$
\begin{aligned}
& \operatorname{dist}\left(\left(\mathbb{1}+h \cdot \mathcal{A}\left(\tau, \mathcal{E}_{\cap}(\tau)\right)\right) x+h \mathcal{B}\left(\tau, \mathcal{E}_{\cap}(\tau)\right) u, \quad \mathcal{E}_{\cap}(\tau+h)\right) \\
& \leq C \cdot \sum_{j=1}^{N} \mathbb{E}\left(\left(\mathbb{1}+h \cdot \mathcal{A}\left(\tau, \mathcal{E}_{\cap}(\tau)\right)\right) E_{j}(\tau)+h \mathcal{B}\left(\tau, \mathcal{E}_{\cap}(\tau)\right) U, \quad E_{j}(\tau+h)\right)
\end{aligned}
$$

with a constant $C>0$ depending on $r_{0}, x_{0}, N, \sup _{\substack{s \in[0, T] \\ k=1, \ldots, N}} \mathbb{d}\left(\{0\}, E_{k}(s)\right)<\infty$. 
Let $H:[\tau, \widetilde{T}] \rightsquigarrow \mathbb{R}^{n}$ denote the unique solution tube of the IVP

$$
\stackrel{\circ}{H}(s)=\mathcal{A}(s, H(s)) x+\mathcal{B}(s, H(s)) U \quad \text { in }[\tau, \widetilde{T}], \quad H(\tau)=\mathcal{E}_{\cap}(\tau) \in \mathcal{K}_{\mathrm{co}}\left(\mathbb{R}^{n}\right)
$$

according to Proposition 2.2. All its values are convex since each $H(s) \subset \mathbb{R}^{n}$ coincides with the reachable set of a nonautonomous linear differential inclusion. For every $s \in[\tau, \widetilde{T}]$, Proposition 2.5 guarantees $\mathbb{d}(K(s), H(s)) \leq \delta(\tau) \cdot e^{\operatorname{const}(\Gamma, \Lambda) \cdot(s-\tau)}$.

Then, $H$ fulfills the integral funnel condition at time $\tau$, i.e.,

$$
\begin{aligned}
0 & =\lim _{h \downarrow 0} \frac{1}{h} \cdot \mathbb{d}\left(H(\tau+h), \bigcup_{x \in H(\tau)}(x+h \cdot(\mathcal{A}(\tau, H(\tau)) x+\mathcal{B}(\tau, H(\tau)) U))\right) \\
& =\lim _{h \downarrow 0} \frac{1}{h} \cdot \mathbb{d}(H(\tau+h),(\mathbb{1}+h \cdot \mathcal{A}(\tau, H(\tau))) H(\tau)+h \cdot \mathcal{B}(\tau, H(\tau)) U) .
\end{aligned}
$$

Indeed, the autonomous differential inclusion $x^{\prime} \in A(\tau, H(\tau)) x+B(\tau, H(\tau)) U$ induces the auxiliary tube $R:[0, \infty) \rightsquigarrow \mathbb{R}^{n}$ of reachable sets of $H(\tau) \subset \mathbb{R}^{n} . R(\cdot)$ satisfies the integral funnel condition at (even) every time $h \in[0, \infty)$ as a consequence of Proposition 2.1. Furthermore, Proposition 2.5 and Assumptions 3.2 (iii), (iv) lead to this upper bound for each $h \in[0, \widetilde{T}-\tau]$

$$
\begin{aligned}
& \mathbb{d}(H(\tau+h), R(h)) \\
& \leq \int_{0}^{h} \sup _{x \in \overline{\mathbb{B}}_{\rho}}\|\mathcal{A}(\tau+s, H(\tau+s)) x-\mathcal{A}(\tau, H(\tau)) x\| \mathrm{d} s \cdot e^{(\Gamma+\Lambda) T} \\
& \leq \int_{0}^{h} \rho\|\mathcal{A}(\tau+s, H(\tau+s))-\mathcal{A}(\tau, H(\tau))\|_{\mathrm{op}} \mathrm{d} s \cdot e^{(\Gamma+\Lambda) T}
\end{aligned}
$$

with $\rho:=e^{\Gamma T} \cdot \sup \left\{\mathbb{d}\left(\{0\}, E_{k}(s) \cup K_{0}\right)+\Gamma T \mid s \in[0, T], k=1, \ldots, N\right\}$. The characterization of $J_{\varepsilon}$ and the continuity of $H(\cdot)$ imply $\lim _{h \downarrow 0} \frac{1}{h} \cdot \mathbb{d}(H(\tau+$ $h), R(h))=0$ and thus,

$$
\begin{aligned}
& \lim _{h \downarrow 0} \frac{1}{h} \cdot \mathbb{d}(H(\tau+h),(\mathbb{1}+h \mathcal{A}(\tau, H(\tau))) H(\tau)+h \mathcal{B}(\tau, H(\tau)) U) \\
& \quad=\lim _{h \downarrow 0} \frac{1}{h} \cdot \mathbb{d}(H(\tau+h),(\mathbb{1}+h \mathcal{A}(\tau, H(\tau))) R(0)+h \mathcal{B}(\tau, H(\tau)) U) \\
& \quad \leq 0+\lim _{h \downarrow 0} \frac{1}{h} \cdot \mathbb{d}(R(h),(\mathbb{1}+h \mathcal{A}(\tau, H(\tau))) R(0)+h \mathcal{B}(\tau, H(\tau)) U)=0,
\end{aligned}
$$

i.e., $H$ satisfies the integral funnel condition at time $\tau$. 
In the next step, we conclude from the triangle inequality for every $h \in(0, \widetilde{T}-t)$

$$
\begin{aligned}
\delta(\tau+h) \stackrel{\text { Def. }}{=} & \mathbb{e}\left(K(\tau+h), \mathcal{E}_{\cap}(\tau+h)\right) \leq \mathbb{d}(K(\tau+h), H(\tau+h))+\mathbb{e}\left(H(\tau+h), \mathcal{E}_{\cap}(\tau+h)\right) \\
\leq & \delta(\tau) \cdot e^{C(\Gamma, \Lambda) \cdot h}+\mathbb{d}(H(\tau+h), \quad(\mathbb{1}+h \cdot \mathcal{A}(\tau, H(\tau))) H(\tau)+h \cdot \mathcal{B}(\tau, H(\tau)) U) \\
& +\mathbb{e}\left((\mathbb{1}+h \cdot \mathcal{A}(\tau, H(\tau))) \mathcal{E}_{\cap}(\tau)+h \cdot \mathcal{B}(\tau, H(\tau)) U, \mathcal{E}_{\cap}(\tau+h)\right)
\end{aligned}
$$

due to $H(\tau)=\mathcal{E}_{\cap}(\tau)$. Thus, we have $\limsup _{h \downarrow 0} \frac{\delta(\tau+h)-\delta(\tau)}{h} \leq C(\Gamma, \Lambda) \cdot \delta(\tau)$ for every $\tau$ in $J_{\varepsilon} \subset\left[t_{0}, \widetilde{T}\right)$. As $\underset{\varepsilon}{\varepsilon} \in\left(0, \widetilde{T}-t_{0}\right)$ had been chosen arbitrarily, the last inequality holds for a.e. $\tau \in\left[t_{0}, \widetilde{T}\right]$ and so, GRONWALL's inequality implies $\delta=0$ in $\left[t_{0}, \widetilde{T}\right]$, i.e., $K(t) \subset \mathcal{E}_{\cap}(t)$ for each $t \in\left[t_{0}, \widetilde{T}\right]$.

Finally, it is worth mentioning that $\widetilde{T}$ was chosen as $\widetilde{T}=\min \left\{t_{0}+\Delta, T\right\}$ with $\Delta>0$ depending only on $r_{0}$ and the LIPSCHITZ constants of $K(\cdot), E_{1}(\cdot), \ldots, E_{N}(\cdot)$ (but not on $t_{0}$ ). As a consequence, we conclude $\delta=0$ in the whole interval $[0, T]$ by means of finitely many subintervals of this form $\left[t_{0}, \widetilde{T}\right]$ (chosen in a piecewise way).

\subsection{A Computational Method for an External Approximation With Ellipsoidal Values (Proposition 3.3)}

On our way to proving Proposition 3.3, we consider the required property $\bigcap_{k=1}^{N} \mathcal{E}\left(x_{k}(t), X_{k}(t)\right) \neq \varnothing$ a technical challenge since its relationship with the ODE systems (for all $\left.x_{j}(\cdot), X_{j}(\cdot)\right)$ is not really obvious. Hence, for $\rho \geq 0$ fixed arbitrarily, we focus on the following auxiliary problem

$$
\begin{aligned}
& \ell_{j}^{\prime}(t)=-\mathcal{A}\left(t, \widetilde{\mathcal{E}}_{\cap, \rho}(t)\right)^{\top} \quad \ell_{j}(t) \\
& x_{j}^{\prime}(t)=\mathcal{A}\left(t, \widetilde{\mathcal{E}}_{\cap, \rho}(t)\right) \quad x_{j}(t)+\mathcal{B}\left(t, \widetilde{\mathcal{E}}_{\cap, \rho}(t)\right) q_{u}, \\
& X_{j}^{\prime}(t)=\mathcal{A}\left(t, \widetilde{\mathcal{E}}_{\cap, \rho}(t)\right) X_{j}(t)+\widetilde{\pi}_{j}(t) X_{j}(t) \\
& \quad+\quad X_{j}(t) \mathcal{A}\left(t, \widetilde{\mathcal{E}}_{\cap, \rho}(t)\right)^{\top}+\frac{1}{\pi_{j}(t)} \widetilde{Q}_{\mathcal{B} \cap}(t)
\end{aligned}
$$

$(j=1, \ldots, N)$ with the modified abbreviations

$$
\begin{aligned}
& \Delta_{1, \rho}(t):=\rho, \Delta_{j, \rho}(t):=\rho+2 \mathrm{~g}\left(\mathcal{E}\left(x_{j}(t), X_{j}(t)\right), \bigcap_{k=1}^{j-1}\left(\mathcal{E}\left(x_{k}(t), X_{k}(t)\right)+\overline{\mathbb{B}}_{\Delta_{k, \rho}(t)}\right)\right) \quad(j \geq 2), \\
& \widetilde{\mathcal{E}}_{\cap, \rho}(t):=\bigcap_{k=1}^{N}\left(\mathcal{E}\left(x_{k}(t), X_{k}(t)\right)+\overline{\mathbb{B}}_{\rho+\Delta_{k, \rho}(t)}\right), \\
& \widetilde{Q}_{\mathcal{B} \cap}(t):=\mathcal{B}\left(t, \widetilde{\mathcal{E}}_{\cap, \rho}(t)\right) Q_{u} \mathcal{B}\left(t, \widetilde{\mathcal{E}}_{\cap, \rho}(t)\right)^{\top} \in \mathbb{R}^{n \times n}, \quad \tilde{\pi}_{j}(t):=\sqrt{\frac{\left\langle\ell_{j}(t), \widetilde{Q}_{\mathcal{B} \cap}(t) \ell_{j}(t)\right\rangle}{\left\langle\ell_{j}(t), X_{j}(t) \ell_{j}(t)\right\rangle}} .
\end{aligned}
$$

In comparison with the original problem (7), (8), the intersection $\mathcal{E}_{\cap}(t) \stackrel{\text { Def. }}{=}$ $\bigcap_{k=1}^{N} \mathcal{E}\left(x_{k}(t), X_{k}(t)\right)$ is replaced by its superset $\widetilde{\mathcal{E}}_{\cap, \rho}(t) \subset \mathbb{R}^{n}$ which has two key properties: 
- $\widetilde{\mathcal{E}}_{\cap, \rho}(t) \neq \emptyset$ is convex. If $\rho>0$, it contains a closed ball of radius $\rho>0$. Indeed, for each $j \in\{2, \ldots, N\}, \mathcal{E}\left(x_{j}(t), X_{j}(t)\right)+\overline{\mathbb{B}}_{\Delta_{j, \rho}(t)}$ contains a ball with some center $z_{j} \in \bigcap_{k=1}^{j-1}\left(\mathcal{E}\left(x_{k}(t), X_{k}(t)\right)+\overline{\mathbb{B}}_{\Delta_{k, \rho}(t)}\right) \neq \emptyset$ and radius $\rho$. Hence, $\overline{\mathbb{B}}_{\rho}\left(z_{j}\right)$ $\subset \bigcap_{k=1}^{j}\left(\mathcal{E}\left(x_{k}(t), X_{k}(t)\right)+\overline{\mathbb{B}}_{\left.\rho+\Delta_{k, \rho}(t)\right)}\right.$.

- If $\mathcal{E}_{\cap}(t) \neq \emptyset$, we have $\Delta_{j, \rho}(t)=\rho$ for all $j \in\{1, \ldots, N\}$ and thus $\mathcal{E}_{\cap}(t) \subset \widetilde{\mathcal{E}}_{\cap, \rho}(t)=\bigcap_{k=1}^{N}\left(\mathcal{E}\left(x_{k}(t), X_{k}(t)\right)+\overline{\mathbb{B}}_{2 \rho}\right)$. It implies $\operatorname{Lim}_{\rho \downarrow} \widetilde{\mathcal{E}}_{\cap, \rho}(t)=$ $\mathcal{E}_{\cap}(t)$.

Lemma 5.8 ( [25, Theorems 2, 3, 5]) Consider any $p_{1}, p_{2} \in \mathbb{R}^{n}$ and positive semidefinite symmetric $Q_{1}, Q_{2} \in \mathbb{R}^{n \times n}$. Set $M:=\max \left\{\sqrt{\left\|Q_{1}\right\|_{\mathrm{op}}}, \sqrt{\left\|Q_{2}\right\|_{\mathrm{op}}}\right\}>0$. Then, the following inequalities hold:

(1.) $\delta_{0}:=\mathbb{d}\left(\mathcal{E}\left(0, Q_{1}\right), \mathcal{E}\left(0, Q_{2}\right)\right), \Delta_{0}:=\sqrt{\left\|Q_{1}-Q_{2}\right\|_{\text {op }}}$ fulfill $\frac{\Delta_{0}^{2}}{M+\sqrt{M^{2}+\Delta_{0}^{2}}} \leq \delta_{0} \leq$ $\Delta_{0} \leq \sqrt{\delta_{0}^{2}+2 M \delta_{0}}$

(2.) $\delta:=\mathbb{d}\left(\mathcal{E}\left(p_{1}, Q_{1}\right), \quad \mathcal{E}\left(p_{2}, Q_{2}\right)\right)$ and $\Delta:=\left\|p_{1}-p_{2}\right\|+\sqrt{\left\|Q_{1}-Q_{2}\right\|_{\text {op }}}$ satisfy $\frac{\Delta^{2}}{2(M+\Delta)} \leq \delta \leq \Delta \leq \delta+\sqrt{\delta^{2}+2 M \delta}$.

(3.) $\delta:=\mathbb{d}\left(\mathcal{E}\left(p_{1}, Q_{1}\right), \mathcal{E}\left(p_{2}, Q_{2}\right)\right), d:=\left\|p_{1}-p_{2}\right\|+\left\|Q_{1}^{\frac{1}{2}}-Q_{2}^{\frac{1}{2}}\right\|_{\text {op }}$ fulfill $\delta \leq d \leq$ $(1+2 \sqrt{2} n(n+2)) \cdot \delta$.

Lemma 5.9 Let $\mathcal{A}:[0, T] \times \mathcal{K}_{c o}\left(\mathbb{R}^{n}\right) \longrightarrow \mathbb{R}^{n \times n}, \mathcal{B}:[0, T] \times \mathcal{K}_{c o}\left(\mathbb{R}^{n}\right) \longrightarrow \mathbb{R}^{n \times n}$ and $U=\mathcal{E}\left(q_{u}, Q_{u}\right) \subset \mathbb{R}^{n}$ satisfy the assumptions of Proposition 3.3. Fix $\rho>0$. For any initial $\ell_{0 j} \in \mathbb{R}^{n} \backslash\{0\}, x_{0 j} \in \mathbb{R}^{n}$ and positive definite $X_{0 j} \in \mathbb{R}^{n \times n}(j=1, \ldots, N)$ given, consider system (10) with abbreviations (11).

Then, there exist $\tau \in(0, T]$ and at least one tuple of solutions $\ell_{j}, x_{j}:[0, \tau] \longrightarrow \mathbb{R}^{n}$, $X_{j}:[0, \tau] \longrightarrow \mathbb{R}^{n \times n}(j=1, \ldots, N)$.

Proof Let $\operatorname{SPD}(n)$ abbreviate the set of all positive definite symmetric matrices in $\mathbb{R}^{n \times n}$. The functions

$$
\begin{aligned}
& \mathbb{R}^{n} \times\left(\operatorname{SPD}(n),\|\cdot\|_{\mathrm{op}}\right) \longrightarrow\left(\mathcal{K}_{\mathrm{co}}\left(\mathbb{R}^{n}\right), \mathbb{d}\right), \quad(q, Q) \longmapsto \mathcal{E}(q, Q), \\
& \mathcal{K}_{\mathrm{co}}\left(\mathbb{R}^{n}\right) \times \mathcal{K}_{\mathrm{co}}\left(\mathbb{R}^{n}\right) \longrightarrow \mathbb{R}, \quad\left(M_{1}, M_{2}\right) \longmapsto \mathrm{g}\left(M_{1}, M_{2}\right) \text {, } \\
& \mathcal{K}_{\mathrm{co}}\left(\mathbb{R}^{n}\right) \times[0, \infty) \longrightarrow \mathcal{K}_{\mathrm{co}}\left(\mathbb{R}^{n}\right), \quad\left(M_{1}, r\right) \longmapsto M_{1}+\overline{\mathbb{B}}_{r}
\end{aligned}
$$

are continuous due to Lemma 5.8 and the triangle inequality of $\mathbb{d}$. For any sets $M_{1}$, $M_{2} \in \mathcal{K}_{\mathrm{co}}\left(\mathbb{R}^{n}\right)$ with $M_{1}^{\circ} \cap M_{2} \neq \emptyset$, Lemma 5.7 (2.) implies that $\mathcal{K}_{\mathrm{co}}\left(\mathbb{R}^{n}\right) \times \mathcal{K}_{\mathrm{co}}\left(\mathbb{R}^{n}\right) \rightsquigarrow$ $\mathbb{R}^{n},\left(N_{1}, N_{2}\right) \mapsto N_{1} \cap N_{2}$ is continuous with nonempty set values close to $\left(M_{1}, M_{2}\right)$. As a consequence, the compositions

$$
\delta: \mathbb{R}^{n} \times \operatorname{SPD}(n) \times \mathcal{K}_{\mathrm{co}}\left(\mathbb{R}^{n}\right) \longrightarrow \mathbb{R},\left(q, Q, M_{2}\right) \longmapsto \rho+2 \cdot \mathrm{g}\left(\mathcal{E}(q, Q), M_{2}\right)
$$

and $\mathbb{R}^{n} \times \operatorname{SPD}(n) \times \mathcal{K}_{\mathrm{co}}\left(\mathbb{R}^{n}\right) \longrightarrow \mathcal{K}_{\mathrm{co}}\left(\mathbb{R}^{n}\right), \quad\left(q, Q, M_{2}\right) \longmapsto \quad(\mathcal{E}(q, Q)+$ $\overline{\mathbb{B}}_{\delta\left(q, Q, M_{2}\right)} \cap M_{2}$ are continuous.

Hence, the right-hand side of the ODE system (10) is continuous w.r.t. state and measurable w.r.t. time. The local existence of solutions results from a well-known theorem in ODE theory (see, e.g., [64, Ch. III, § 10.XVIII Theorem]). 
Lemma 5.10 Under the assumptions of Proposition 3.3, consider solutions $\ell_{j}$ : $[0, \tau] \longrightarrow \mathbb{R}^{n}, x_{j}:[0, \tau] \longrightarrow \mathbb{R}^{n}, X_{j}:[0, \tau] \longrightarrow \mathbb{R}^{n \times n}(j=1,2)$ to system (10) with abbreviations (11), $\rho>0, \tau \in(0, T]$. Then, it holds for every $t \in[0, \tau]$ and $j \in\{1, \ldots, N\}$ :

(1.) $0<\left\|\ell_{0 j}\right\| e^{-\Gamma t} \leq\left\|\ell_{j}(t)\right\| \leq\left\|\ell_{0 j}\right\| e^{\Gamma t}$ and $\left\|x_{j}(t)\right\| \leq \operatorname{const}(\Gamma)$. $\left(\left\|x_{0 j}\right\|+\left\|q_{u}\right\| t\right) \cdot e^{\Gamma t}$

(2.) $X_{j}(t) \in \mathbb{R}^{n \times n}$ is symmetric.

(3.) There is $\gamma_{j}>0$ (depending on $X_{0 j} \in \mathbb{R}^{n \times n}$ ) such that for all $v \in \mathbb{R}^{n}$, $\gamma_{j} e^{-2 \Gamma t}\|v\|^{2} \leq\left\langle v, X_{j}(t) v\right\rangle$. Hence, $X_{j}(t) \in \mathbb{R}^{n \times n}$ is positive definite.

(4.) There exist $\widetilde{c}_{j}, \widetilde{C}_{j}>0$ (depending on $\Gamma, Q_{u}$ ) and $c_{j}, C_{j}>0$ (depending on $\Gamma$, $\left.Q_{u}, X_{0 j}\right)$ with

$$
\begin{aligned}
\widetilde{c}_{j}\left\|\ell_{0 j}\right\|^{2} e^{-2 \Gamma t} & \leq\left\langle\ell_{j}(t), \widetilde{Q}_{\left.\mathcal{B} \cap(t) \ell_{j}(t)\right\rangle} \leq \widetilde{C}_{j}\left\|\ell_{0 j}\right\|^{2} e^{2 \Gamma t}\right. \\
0<c_{j} e^{-2 \Gamma t} & \leq \widetilde{\pi}_{j}(t) \\
& \leq C_{j} e^{2 \Gamma t} .
\end{aligned}
$$

(5.) $\left\|X_{j}(t)\right\|_{\text {op }} \leq \operatorname{const}\left(\Gamma, Q_{u}, X_{0 j}, T\right) \cdot e^{2 \Gamma t},\left\|X_{j}^{\prime}(t)\right\|_{\text {op }} \leq \operatorname{const}\left(\Gamma, Q_{u}, X_{0 j}, T\right)$. $e^{4 \Gamma t}$.

As a consequence of the a priori bounds (1.), (5.), each solution tuple to the IVP with ODE system (10) and $\rho>0$ can be extended to $[0, T]$ (see, e.g., [64, Ch. III, $\S 10 . X X$ Theorem]).

Proof (1.) $\quad \ell_{j}(\cdot)$ solves the adjoint ODE $\ell_{j}^{\prime}(t)=-\mathcal{A}\left(t, \widetilde{\mathcal{E}}_{\cap, \rho}(t)\right)^{\top} \ell_{j}(t)$ in $[0, \tau]$ and, $\mathcal{A}^{\top}$ is bounded by $\Gamma$ due to hypothesis 3.3 (iv'). $x_{j}(\cdot)$ is characterized as the solution to an inhomogeneous linear ODE and so, GRONWALL's inequality provides an explicit a priori bound.

(2.) $Q_{u} \in \mathbb{R}^{n \times n}$ is symmetric by assumption and so is $\widetilde{Q}_{\mathcal{B} \cap}(t) \in \mathbb{R}^{n \times n}$ then. Hence, both $X_{j}(\cdot)$ and $X_{j}(\cdot)^{\top}$ are CARATHÉODORY solutions $Y:[0, \tau] \longrightarrow \mathbb{R}^{n \times n}$ to the ODE $Y^{\prime}(t)=\mathcal{A}\left(t, \widetilde{\mathcal{E}}_{\cap, \rho}(t)\right) Y(t)+\tilde{\pi}_{j}(t) Y(t)+Y(t) \mathcal{A}\left(t, \widetilde{\mathcal{E}}_{\cap, \rho}(t)\right)^{\top}+\frac{1}{\tilde{\pi}_{j}(t)} \widetilde{Q}_{\mathcal{B} \cap}(t)$ with the same $\tilde{\pi}_{j}(\cdot) . X_{j}(0)=X_{0 j}=X_{j}(0)^{\top}$ implies $X_{j}(t)=X_{j}(t)^{\top}$ for every $t \in[0, \tau]$.

(3.) Choose $t \in(0, \tau]$ and $v_{t} \in \mathbb{R}^{n} \backslash\{0\}$ arbitrarily and let $v:[0, t] \longrightarrow \mathbb{R}^{n}$ denote the unique solution of the adjoint ODE $v^{\prime}=-\mathcal{A}\left(\cdot, \widetilde{\mathcal{E}}_{\cap, \rho}(\cdot)\right)^{\top} v$ with $v(t)=v_{t}$. We conclude from ODE system (10)

$$
\begin{aligned}
\frac{\mathrm{d}}{\mathrm{d} s}\left\langle v(s), X_{j}(s) v(s)\right\rangle & =v(s)^{\top}\left(\tilde{\pi}_{j}(s) X_{j}(s)+\frac{1}{\tilde{\pi}_{j}(s)} \widetilde{Q}_{\mathcal{B} \cap(s)) v(s)}\right. \\
& =\tilde{\pi}_{j}(s)\left\langle v(s), X_{j}(s) v(s)\right\rangle+\frac{1}{\tilde{\pi}_{j}(s)}\left\langle v(s), \widetilde{Q}_{\mathcal{B} \cap}(s) v(s)\right\rangle
\end{aligned}
$$

at a.e. time $s \in[0, \tau)$ and hence, $\frac{\mathrm{d}}{\mathrm{d} s}\left\langle v(s), X_{j}(s) v(s)\right\rangle \geq 0$. At time $s=t$, in particular, we obtain

$$
\begin{aligned}
& \left\langle v_{t}, X_{j}(t) v_{t}\right\rangle=\left\langle v(t), X_{j}(t) v(t)\right\rangle \geq\left\langle v(0), X_{0 j} v(0)\right\rangle \geq \gamma_{j}\|v(0)\|^{2} \\
& \geq \gamma_{j} e^{-2 \Gamma t}\left\|v_{t}\right\|^{2}>0
\end{aligned}
$$


with $\gamma_{j}=\operatorname{const}\left(X_{0 j}\right)>0$, i.e., $X_{j}(t) \in \mathbb{R}^{n \times n}$ is positive definite.

(4.) For every $t \in[0, \tau]$, the CAUCHY-SCHWARZ inequality and assumption 3.3 (iv') lead to

$$
\begin{aligned}
\left\langle\ell_{j}(t), \widetilde{Q}_{\mathcal{B} \cap}(t) \ell_{j}(t)\right\rangle & \leq\left\|Q_{u}\right\|\left\|\mathcal{B}\left(t, \mathcal{E}_{\cap}(t)\right)^{\top}\right\|_{\text {op }}^{2}\left\|\ell_{j}(t)\right\|^{2} \\
& \leq\left\|Q_{u}\right\| \Gamma^{2} \cdot\left\|\ell_{0 j}\right\|^{2} e^{2 \Gamma t} .
\end{aligned}
$$

Similarly, we conclude from Eq. (11), $Q_{u} \in \mathbb{R}^{n \times n}$ being positive definite and hypotheses 3.3 (iv'), (vi')

$$
\begin{aligned}
\left\langle\ell_{j}(t), \widetilde{Q}_{\mathcal{B} \cap}(t) \ell_{j}(t)\right\rangle & =\left\langle\mathcal{B}\left(t, \widetilde{\mathcal{E}}_{\cap, \rho}(t)\right)^{\top} \ell_{j}(t), \quad Q_{u} \mathcal{B}\left(t, \widetilde{\mathcal{E}}_{\cap, \rho}(t)\right)^{\top} \ell(t)\right\rangle \\
& \geq \operatorname{const}\left(Q_{u}\right)\left\|\mathcal{B}\left(t, \widetilde{\mathcal{E}}_{\cap, \rho}(t)\right)^{\top} \ell_{j}(t)\right\|^{2} \\
& \geq \operatorname{const}\left(\Gamma, Q_{u}\right)\left\|\ell_{0 j}\right\|^{2} e^{-2 \Gamma t} .
\end{aligned}
$$

In regard to the other scalar product in the quotient $\pi_{j}(t)$, we have already verified in the proof of the statement (3.) that $[0, \tau] \longrightarrow \mathbb{R}, t \longmapsto\left\langle\ell_{j}(t), X_{j}(t) \ell_{j}(t)\right\rangle$ is non-decreasing and so, for every $t \in[0, \tau]$,

$$
\left\langle\ell_{j}(t), X_{j}(t) \ell_{j}(t)\right\rangle \geq\left\langle\ell_{j}(0), X_{0 j} \ell_{j}(0)\right\rangle \geq \operatorname{const}\left(X_{0 j}\right)\left\|\ell_{0 j}\right\|^{2} .
$$

Furthermore, its time derivative mentioned there implies for a.e. $t \in[0, \tau]$

$$
\begin{aligned}
\frac{\mathrm{d}}{\mathrm{d} t} \sqrt{\left\langle\ell_{j}(t), X_{j}(t) \ell_{j}(t)\right\rangle} & =\frac{1}{2 \sqrt{\left\langle\ell_{j}(t), X_{j}(t) \ell_{j}(t)\right\rangle}} \cdot \frac{\mathrm{d}}{\mathrm{d} t}\left\langle\ell_{j}(t), \quad X_{j}(t) \ell_{j}(t)\right\rangle \\
& =\sqrt{\left\langle\mathcal{B}\left(t, \widetilde{\mathcal{E}}_{\cap, \rho}(t)\right)^{\top} \ell_{j}(t), \quad Q_{u} \mathcal{B}\left(t, \widetilde{\mathcal{E}}_{\cap, \rho}(t)\right)^{\top} \ell_{j}(t)\right\rangle} .
\end{aligned}
$$

Assumption 3.3 (iv') (again) guarantees for a.e. $t \in[0, \tau]$

$$
\frac{\mathrm{d}}{\mathrm{d} t} \sqrt{\left\langle\ell_{j}(t), X_{j}(t) \ell_{j}(t)\right\rangle} \leq \operatorname{const}\left(\Gamma, Q_{u}\right)\left\|\ell_{j}(t)\right\| \leq \operatorname{const}\left(\Gamma, Q_{u}\right) e^{\Gamma t}\left\|\ell_{0 j}\right\|
$$

and thus for all $t \in[0, \tau]$

$$
\begin{aligned}
& \sqrt{\left\langle\ell_{j}(t), X_{j}(t) \ell_{j}(t)\right\rangle} \\
& \leq \operatorname{const}\left(\Gamma, Q_{u}\right)\left\|\ell_{0 j}\right\|\left(1+\int_{0}^{t} e^{\Gamma s} \mathrm{~d} s\right) \leq \operatorname{const}\left(\Gamma, Q_{u}\right)\left\|\ell_{0 j}\right\| e^{\Gamma t} .
\end{aligned}
$$

(5.) In combination with Assumptions 3.3 (iv'), (vi'), ODE (10) of $X_{j}(\cdot)$ has the consequence

$$
\begin{aligned}
& \left\|X_{j}^{\prime}(t)\right\|_{\text {op }} \leq \Gamma\left\|X_{j}(t)\right\|_{\text {op }} \\
& \quad+\left\|X_{j}(t)\right\|_{\text {op }}\left\|\mathcal{A}\left(t, \widetilde{\mathcal{E}}_{\cap, \rho}(t)\right)^{\top}\right\|_{\text {op }}+\tilde{\pi}_{j}(t)\left\|X_{j}(t)\right\|_{\text {op }}+\frac{1}{\tilde{\pi}_{j}(t)}\left\|\widetilde{Q}_{\mathcal{B} \cap}(t)\right\|_{\text {op }}
\end{aligned}
$$




$$
\begin{aligned}
\leq & 2 \Gamma\left\|X_{j}(t)\right\|_{\text {op }}+C_{j} e^{2 \Gamma t}\left\|X_{j}(t)\right\|_{\text {op }} \\
& +\frac{1}{c_{j}} e^{2 \Gamma t}\left\|\widetilde{Q}_{\mathcal{B} \cap}(t)\right\|_{\text {op }} \\
\leq & \operatorname{const}\left(\Gamma, Q_{u}, X_{0 j}\right) \cdot e^{2 \Gamma t}\left\|X_{j}(t)\right\|_{\text {op }}+\frac{1}{c_{j}} e^{2 \Gamma t} \Gamma^{2}\left\|Q_{u}\right\|_{\text {op }}
\end{aligned}
$$

for a.e. $t \in[0, \tau]$. GRONWALL's inequality provides $\left\|X_{j}(t)\right\|_{\text {op }} \leq \operatorname{const}\left(\Gamma, Q_{u}\right.$, $\left.X_{0 j}, T\right) \cdot\left(\left\|X_{0 j}\right\|_{\text {op }}+\left\|Q_{u}\right\|_{\text {op }}\right) \cdot e^{2 \Gamma t}$ for all $t \in[0, \tau] \subset[0, T]$.

Subsequent Lemma 5.12 proves the aspect of existence in Proposition 3.3 (1.).

Afterward, the statement about uniqueness follows in Lemma 5.14. In preparation for both results, the next lemma indicates a technically essential property of solutions to the "approximating" system (i.e., with $\rho>0$ ) and the "exact" system (i.e., $\rho=0$ ).

Lemma 5.11 In addition to the assumptions of Proposition 3.3, fix $\rho \geq 0$ arbitrarily and let $\ell_{j}:[0, T] \longrightarrow \mathbb{R}^{n}, x_{j}:[0, T] \longrightarrow \mathbb{R}^{n}, X_{j}:[0, T] \longrightarrow \mathbb{R}^{n \times n}(j=$ $1, \ldots, N)$ be solutions to ODE system (10) with initial values $\ell_{0 j} \in \mathbb{R}^{n} \backslash\{0\}, x_{0 j} \in \mathbb{R}^{n}$ and positive definite symmetric $X_{0 j} \in \mathbb{R}^{n \times n}$ given such that $\bigcap_{k=1}^{N} \mathcal{E}\left(x_{0 k}, X_{0 k}\right) \subset \mathbb{R}^{n}$ has nonempty interior.

Then, there exists a radius $\widehat{r}=\widehat{r}\left(\Gamma, T, x_{0} ., X_{0}.\right)>0$ such that for every $t \in[0, T]$, the intersection $\mathcal{E}_{\cap}(t) \stackrel{\text { Def. }}{=} \bigcap_{k=1}^{N} \mathcal{E}\left(x_{k}(t), X_{k}(t)\right)$ contains a ball with radius $\widehat{r}$.

Proof $\mathcal{E}_{\cap}(0) \stackrel{\text { Def. }}{=} \bigcap_{k=1}^{N} \mathcal{E}\left(x_{0 k}, X_{0 k}\right) \subset \mathbb{R}^{n}$ has nonempty interior and so, we can choose $z_{0} \in \mathbb{R}^{n}$ and $R_{0}>0$ with $\overline{\mathbb{B}}_{R_{0}}\left(z_{0}\right) \subset \mathcal{E}_{\cap}(0)$. Set $\widehat{r}:=R_{0} e^{-\Gamma T}>0$. Let $z:[0, T] \longrightarrow$ $\mathbb{R}^{n}$ denote the unique solution of

$$
z^{\prime}=\mathcal{A}\left(\cdot, \widetilde{\mathcal{E}}_{\cap, \rho}(\cdot)\right) z+\mathcal{B}\left(\cdot, \widetilde{\mathcal{E}}_{\cap, \rho}(\cdot)\right) q_{u} \quad \text { in }[0, T], \quad z(0)=z_{0}
$$

Then, $\overline{\mathbb{B}}_{\widehat{r}}(z(t)) \subset \mathbb{R}^{n}$ is contained in the reachable set $R(t)$ of the differential inclusion $x^{\prime} \in \mathcal{A}\left(\cdot, \widetilde{\mathcal{E}}_{\cap, \rho}(\cdot)\right) x+\mathcal{B}\left(\cdot, \widetilde{\mathcal{E}}_{\cap, \rho}(\cdot)\right) U$ and the initial set $\overline{\mathbb{B}}_{R_{0}}\left(z_{0}\right)$ at each time $t \in[0, T]$. Indeed, for each $y_{t} \in \overline{\mathbb{B}}_{\widehat{r}}(z(t))$, the variation of constants formula provides the solution $y:[0, t] \longrightarrow \mathbb{R}^{n}$ of $y^{\prime}=\mathcal{A}\left(\cdot, \widetilde{\mathcal{E}}_{\cap, \rho}(\cdot)\right) y+\mathcal{B}\left(\cdot, \widetilde{\mathcal{E}}_{\cap, \rho}(\cdot)\right) q_{u}$ with $y(t)=y_{t}$ and, GRONWALL's inequality implies

$$
\left\|y(0)-z_{0}\right\|=\|y(0)-z(0)\| \leq\|y(t)-z(t)\| \cdot e^{\Gamma t} \leq \widehat{r} \cdot e^{\Gamma t}=R_{0}
$$

i.e., $y_{t} \in R(t)$. Due to Assumptions 3.2 (iii) and 3.3 (ii'), the compositions $\mathcal{A}\left(\cdot, \widetilde{\mathcal{E}}_{\cap, \rho}(\cdot)\right), \mathcal{B}\left(\cdot, \widetilde{\mathcal{E}}_{\cap, \rho}(\cdot)\right):[0, T] \longrightarrow \mathbb{R}^{n \times n}$ are continuous. Hence, we can conclude from Proposition 3.1 (2.) that $R(t)$ is a subset of each $\mathcal{E}\left(x_{j}(t), X_{j}(t)\right)$ $(j=1, \ldots, N)$, i.e., $\overline{\mathbb{B}}_{\widehat{r}}(z(t)) \subset \mathcal{E}_{\cap}(t)$.

Lemma 5.12 Suppose the assumptions of Proposition 3.3.

For all initial $\ell_{0 j} \in \mathbb{R}^{n} \backslash\{0\}, x_{0 j} \in \mathbb{R}^{n}$ and positive definite $X_{0 j} \in \mathbb{R}^{n \times n}(j=$ $1, \ldots, N)$ given, there exist solutions $\ell_{j}:[0, T] \longrightarrow \mathbb{R}^{n}, x_{j}:[0, T] \longrightarrow \mathbb{R}^{n}, X_{j}$ : 
$[0, T] \longrightarrow \mathbb{R}^{n \times n}(j=1, \ldots, N)$ to system (10) with $\rho=0$ satisfying for every $t \in[0, T]$

- $\mathcal{E}_{\cap}(t) \stackrel{\text { Def. }}{=} \bigcap_{k=1}^{N} \mathcal{E}\left(x_{k}(t), X_{k}(t)\right)$ has nonempty interior.

- $\ell_{j}(t) \neq 0$ and $X_{j}(t) \in \mathbb{R}^{n \times n}$ is symmetric.

- There exist $\widetilde{c}_{j}, \widetilde{C}_{j}>0$ (depending on $\Gamma, Q_{u}$ ) and $c_{j}, C_{j}>0$ (depending on $\Gamma$, $\left.Q_{u}, X_{0 j}\right)$ with

$$
\begin{array}{rlrl}
\left\|\ell_{0 j}\right\|^{2} \cdot \widetilde{c}_{j} e^{-2 \Gamma t} & \leq\left\langle\ell_{j}(t), \widetilde{Q}_{\left.\mathcal{B} \cap(t) \ell_{j}(t)\right\rangle} \leq \widetilde{C}_{j} e^{2 \Gamma t}\left\|\ell_{0 j}\right\|^{2}\right. \\
c_{j} e^{-2 \Gamma t} & \leq \pi_{j}(t) & \leq C_{j} e^{2 \Gamma t} \\
\|v\|^{2} \cdot c_{j} e^{-2 \Gamma t} & \leq\left\langle v, X_{j}(t) v\right\rangle & \text { for all } \quad v \in \mathbb{R}^{n} .
\end{array}
$$

- $X_{j}(\cdot)$ is LIPSCHITZ continuous with a LIPSCHITZ constant depending only on $\Gamma$, $Q_{u}, X_{0 j}, T$.

Proof Let $\left(\rho_{\iota}\right)_{\iota \in \mathbb{N}}$ denote any sequence in $(0,1)$ tending to 0 . For each $\iota \in \mathbb{N}$, Lemma 5.9 provides solutions $\ell_{j, \iota}, x_{j, \iota}, X_{j, \iota}(j=1, \ldots, N)$ to ODE system (10) with $\rho=\rho_{\iota}>0$. They exist in the whole interval $[0, T]$ due to Lemma 5.10 (1.),(5.). The a priori estimates in Lemma 5.10 do not depend on $\rho>0$ and thus, they are uniform w.r.t. $\iota \in \mathbb{N}$. Hence, the $3 N$ sequences $\left(\ell_{j, l}\right)_{\iota \in \mathbb{N}},\left(x_{j, l}\right)_{\iota \in \mathbb{N}},\left(X_{j, l}\right)_{\iota \in \mathbb{N}}$ are bounded uniformly and equi-continuous. The compactness theorem of ARZELA-ASCOLI guarantees a joint sequence $\iota_{\kappa} \nearrow \infty$ of indices and continuous functions $\ell_{j}:[0, T] \longrightarrow \mathbb{R}^{n}$, $x_{j}:[0, T] \longrightarrow \mathbb{R}^{n}, X_{j}:[0, T] \longrightarrow \mathbb{R}^{n \times n}(j=1, \ldots, N)$ such that $\ell_{j, \iota_{\kappa}} \longrightarrow \ell_{j}, \quad x_{j, \iota_{\kappa}} \longrightarrow x_{j}, \quad X_{j, \iota_{\kappa}} \longrightarrow X_{j} \quad$ uniformly in $[0, T] \quad(\kappa \longrightarrow \infty)$ (see, e.g., [64, Ch. II, § 7.IV Theorem]). We conclude from Lemma 5.8 (2.)

$$
\mathbb{d}\left(\mathcal{E}\left(x_{j, \iota_{\kappa}}(t), X_{j, \iota_{\kappa}}(t)\right), \quad \mathcal{E}\left(x_{j}(t), X_{j}(t)\right)\right) \longrightarrow 0 \quad(\kappa \longrightarrow \infty)
$$

for each $j \in\{1, \ldots, N\}$ and $t \in[0, T]$. Lemma 5.11 provides a radius $\widehat{r}=$ $\widehat{r}\left(\Gamma, T, x_{0} ., X_{0}\right)^{\prime}>0$ and a sequence $\left(z_{\iota}(t)\right)_{\iota \in \mathbb{N}}$ of time-dependent centers in $\mathbb{R}^{n}$ satisfying $\overline{\mathbb{B}}_{\widehat{r}}\left(z_{\iota}(t)\right) \subset \bigcap_{j=1}^{N} \mathcal{E}\left(x_{j, \iota}(t), X_{j, \iota}(t)\right)$ for all $\iota \in \mathbb{N}$ and $t \in[0, T]$. Then, at each time $t \in[0, T]$, a subsequence of $\left(z_{\iota_{\kappa}}(t)\right)_{\kappa \in \mathbb{N}}$ converges to some $z(t) \in \mathbb{R}^{n}$. The convergence in (12) implies $\overline{\mathbb{B}}_{\widehat{r} / 2}(z(t)) \subset \mathcal{E}_{\cap}(t)$. In particular, $\mathcal{E}_{\cap}(t)$ has nonempty interior (as claimed).

Next we conclude from Lemma 5.7 (1.) for every $t \in[0, T]$

$$
\begin{aligned}
& \mathbb{d}\left(\bigcap_{j=1}^{N} \mathcal{E}\left(x_{j, \iota_{\kappa}}(t), X_{j, \iota_{\kappa}}(t)\right), \mathcal{E}_{\cap}(t)\right) \\
& \quad \leq \operatorname{const}(\widehat{r}) \cdot \sum_{j=1}^{N} \mathbb{d}\left(\mathcal{E}\left(x_{j, \iota_{\kappa}}(t), X_{j, \iota_{\kappa}}(t)\right), \mathcal{E}\left(x_{j}(t), X_{j}(t)\right)\right) \stackrel{\kappa \rightarrow \infty}{\longrightarrow} 0 .
\end{aligned}
$$

Thus, the intersections $\widetilde{\mathcal{E}}_{\cap, \rho_{\left(\iota_{K}\right)}}(t)$ used in the coefficients of ODE system (10) for $\rho=\rho_{\left(\iota_{\kappa}\right)}$ converge to $\mathcal{E}_{\cap}(t)$ for $\kappa \longrightarrow \infty$ at each time $t \in[0, T]$. Assumption 3.2 (iii) leads to 
$\lim _{\kappa \rightarrow \infty} \mathcal{A}\left(t, \widetilde{\mathcal{E}}_{\cap, \rho_{\left({ }_{k}\right)}}(t)\right)=\mathcal{A}\left(t, \mathcal{E}_{\cap}(t)\right), \quad \lim _{\kappa \rightarrow \infty} \mathcal{B}\left(t, \widetilde{\mathcal{E}}_{\cap, \rho_{({ })}}(t)\right)=\mathcal{B}\left(t, \mathcal{E}_{\cap}(t)\right)$

CARATHÉODORY solutions to ODEs are characterized by an integral condition at each time $t$ implying the absolute continuity. Due to the uniform bounds in Lemma 5.10, the respective criteria for each index $\iota_{\kappa} \in \mathbb{N}$ and their limits for $\kappa \longrightarrow \infty$ reveal that $\ell_{j}(\cdot), x_{j}(\cdot), X_{j}(\cdot)(j=1, \ldots, N)$ are absolutely continuous in $[0, T]$ and solve the ODE system (10) (with $\rho=0$ ).

Lemma 5.13 ([9] [29, Theorem 6.2]) If the HERMITIAN matrices $A, B \in \mathbb{C}^{n \times n}$ are positive definite then for any unitarily invariant norm, it holds $\left\|A^{\frac{1}{2}}-B^{\frac{1}{2}}\right\| \leq$ $\frac{1}{\sqrt{\lambda_{\min }(A)}+\sqrt{\lambda_{\min }(B)}}\|A-B\|$ where $\lambda_{\min }$ denotes the smallest eigenvalue.

Lemma 5.14 Under the assumptions of Proposition 3.3, let $\ell_{j}:[0, T] \longrightarrow \mathbb{R}^{n}, x_{j}$ : $[0, T] \longrightarrow \mathbb{R}^{n}, X_{j}:[0, T] \longrightarrow \mathbb{R}^{n \times n}(j=1, \ldots, N)$ be solutions to ODE system (7) with initial values $\ell_{0 j} \in \mathbb{R}^{n} \backslash\{0\}, x_{0 j} \in \mathbb{R}^{n}$ and positive definite symmetric $X_{0 j} \in$ $\mathbb{R}^{n \times n}$ such that $\bigcap_{k=1}^{N} \mathcal{E}\left(x_{0 k}, X_{0 k}\right) \subset \mathbb{R}^{n}$ has nonempty interior.

Then, there does not exist any other solution to this IVP in $[0, T]$.

Proof Every solution tuple to an IVP with ODE system (7) also solves the ODE system (10) with $\rho=0$ and abbreviations (11). Due to GRONWALL's inequality, it is sufficient to prove that the functions on the right-hand side of ODE system (10) are LIPSCHITZ continuous w.r.t. states in a neighborhood of the solution values. In comparison with the uniqueness result in Proposition 2.2, for example, it is worth mentioning that now the states are in the vector spaces

- $\mathbb{R}^{n}$ for the directions $\ell_{j}(t)$ and the centers $x_{j}(t)(j=1, \ldots, N)$

- $\mathbb{R}^{n \times n}$ for the matrices $X_{j}(t) \in \mathbb{R}^{n \times n}$ of ellipsoids $(j=1, \ldots, N)$,

but not in the metric space $\mathcal{K}\left(\mathbb{R}^{n}\right)$ (for the ellipsoids).

In contrast to Lemma 5.10, we now assume the existence of solutions in $[0, T]$ as well as (implicitly) $\bigcap_{k=1}^{N} \mathcal{E}\left(x_{k}(t), X_{k}(t)\right) \neq \emptyset$ for all $t \in[0, T]$. Hence, the same arguments (as for Lemma 5.10 (3.),(4.)) lead to constants $\widetilde{c}_{j}, \widetilde{C}_{j}, c_{j}, C_{j}=\operatorname{const}\left(\Gamma, Q_{u}, X_{0 j}\right)>0$ such for $j=1, \ldots, N$ and all $t \in[0, T], v \in \mathbb{R}^{n}$

$$
\begin{aligned}
\left\|\ell_{0 j}\right\|^{2} \cdot \tilde{c}_{j} e^{-2 \Gamma t} & \leq\left\langle\ell_{j}(t), \widetilde{Q}_{\mathcal{B} \cap}(t) \ell_{j}(t)\right\rangle \\
c_{j} e^{-2 \Gamma t} & \leq \widetilde{C}_{j} e^{2 \Gamma t} \| \ell_{j}(t) \\
\|v\|_{0 j} \|^{2} & \leq C_{j} e^{2 \Gamma t} \\
c_{j} e^{-2 \Gamma t} & \leq\left\langle v, X_{j}(t) v\right\rangle .
\end{aligned}
$$

The smallest eigenvalue of $X_{j}(t) \in \mathbb{R}^{n \times n}(t \in[0, T], j \in\{1, \ldots, N\})$ is bounded from below by $c_{j} e^{-2 \Gamma T}>0$. Furthermore, $x_{j}(\cdot)$ and $X_{j}(\cdot)$ are LIPSCHITZ continuous with a LIPSCHITZ constant depending only on $\Gamma, Q_{u}, x_{0 j}, X_{0 j}, T$. Hence, there exists an open neighborhood $\mathcal{U} \subset\left(\mathbb{R}^{n \times n},\|\cdot\|_{\text {op }}\right)$ of the compact set $\left\{X_{j}(t) \mid t \in[0, T], j \in\{1, \ldots, N\}\right\}$ such that each symmetric matrix in $\mathcal{U}$ has eigenvalues $>\min _{j} \frac{c_{j}}{2} e^{-2 \Gamma T}>0$. According to Lemmas 5.8 (3.) and 5.13, all symmetric $Q_{1}, Q_{2} \in \mathcal{U}$ are positive definite and satisfy for every $p_{1}, p_{2} \in \mathbb{R}^{n}$ 


$$
\begin{aligned}
& \mathbb{d}\left(\mathcal{E}\left(p_{1}, Q_{1}\right), \mathcal{E}\left(p_{2}, Q_{2}\right)\right) \leq\left\|p_{1}-p_{2}\right\|+\left\|Q_{1}^{\frac{1}{2}}-Q_{2}^{\frac{1}{2}}\right\|_{\text {op }} \\
& \leq\left\|p_{1}-p_{2}\right\|+\operatorname{const}\left(\Gamma, \min _{j} c_{j}, T\right) \cdot\left\|Q_{1}-Q_{2}\right\|_{\text {op }} .
\end{aligned}
$$

Lemma 5.11 guarantees some $\widehat{r}>0$ such that for all $t \in[0, T]$, a ball with radius $\widehat{r}$ is contained in $\bigcap_{j=1}^{N} \mathcal{E}\left(x_{j}(t), X_{j}(t)\right)$.

There exists an open neighborhood $\mathcal{V} \subset\left(\mathbb{R}^{n} \times \mathbb{R}^{n \times n}\right)^{N}$ of the compact set $\left\{\left(x_{k}(t), X_{k}(t)\right)_{k=1, \ldots, N} \mid t \in[0, T]\right\}$ such that for all $j \in\{1, \ldots, N\}, p_{j} \in \mathbb{R}^{n}$ and symmetric $Q_{j} \in \mathbb{R}^{n \times n}$ with $\left(p_{k}, Q_{k}\right)_{k=1, \ldots, N} \in \mathcal{V}$,

- $Q_{j} \in \mathcal{U} \subset \mathbb{R}^{n \times n}$ for each $j \in\{1, \ldots, N\}$ and

- $\bigcap_{j=1}^{N} \mathcal{E}\left(p_{j}, Q_{j}\right) \subset \mathbb{R}^{n}$ contains a ball of radius $\frac{\hat{r}}{2}$.

Lemma 5.7 (1.) and the characterization of $\mathcal{U}$ imply for all $\left(p_{k}, Q_{k}\right)_{k=1, \ldots, N}$, $\left(\widetilde{p}_{k}, \widetilde{Q}_{k}\right)_{k=1, \ldots, N} \in \mathcal{V}$

$$
\begin{aligned}
\mathbb{d}\left(\bigcap_{k=1}^{N} \mathcal{E}\left(p_{k}, Q_{k}\right), \bigcap_{k=1}^{N} \mathcal{E}\left(\tilde{p}_{k}, \widetilde{Q}_{k}\right)\right) & \leq \mathrm{const} \cdot \sum_{k=1}^{N} \mathbb{d}\left(\mathcal{E}\left(p_{k}, Q_{k}\right), \mathcal{E}\left(\widetilde{p}_{k}, \widetilde{Q}_{k}\right)\right) \\
& \leq \text { const } \cdot \sum_{k=1}^{N}\left(\left\|p_{k}-\tilde{p}_{k}\right\|+\left\|Q_{k}-\widetilde{Q}_{k}\right\|_{\text {op }}\right) .
\end{aligned}
$$

Together with Assumptions 3.2 (ii), (iii) and 3.3 (iv'),(vi'), this inequality leads to all the aspects of (local) LIPSCHITZ continuity w.r.t. state required for concluding the claimed uniqueness of solutions to ODE system (10) from GRONWALL's inequality.

Finally, we prove Proposition 3.3 (2.) in form of Lemma 5.16 below:

Lemma 5.15 ([33, $\S 2.1$ and Lemma 2.2.1 (a)]) Let $\mathcal{E}\left(p_{1}, Q_{1}\right)$ and $\mathcal{E}\left(p_{2}, Q_{2}\right)$ be ellipsoids in $\mathbb{R}^{n}$. Then, these statements hold:

(1.) for all $A \in \mathbb{R}^{n \times n}, b \in \mathbb{R}^{n}, A \mathcal{E}\left(p_{1}, Q_{1}\right)+b=\mathcal{E}\left(A p_{1}+b, A Q_{1} A^{\top}\right)$,

(2.) $\mathcal{E}\left(p_{1}, Q_{1}\right)+\mathcal{E}\left(p_{2}, Q_{2}\right) \subset \mathcal{E}\left(p_{1}+p_{2},\left(1+\frac{1}{\eta}\right) Q_{1}+(1+\eta) Q_{2}\right)$ for all $\eta>0$.

Lemma 5.16 Under the assumptions of Proposition 3.3 consider any solutions $\ell_{j}(\cdot)$, $x_{j}(\cdot), X_{j}(\cdot)(j=1, \ldots, N)$ to ODE system (7) with the additional features mentioned in Lemma 5.12 .

Then, $E_{j}:[0, T] \rightsquigarrow \mathbb{R}^{n}, t \mapsto \mathcal{E}\left(x_{j}(t), X_{j}(t)\right)(j=1, \ldots, N)$ have the following properties:

(1.) $E_{j}(\cdot)$ is LIPSCHITZ continuous (w.r.t. $\left.\mathbb{d}\right)$.

(2.) For every $t \in[0, T), \quad 0=\lim _{h \downarrow 0} \frac{1}{h} \cdot \mathbb{e}\left(\left(\mathbb{1}+h \mathcal{A}\left(t, \mathcal{E}_{\cap}(t)\right)\right) E_{j}(t)+\right.$ $\left.h \mathcal{B}\left(t, \mathcal{E}_{\cap}(t)\right) U, \quad E_{j}(t+h)\right)$. 
Proof (1.) $\quad X_{j}(\cdot)$ is LIPSCHITZ continuous and, there exists $\gamma_{j}=\gamma_{j}\left(X_{0 j}\right)>0$ such that $\left\langle v, X_{j}(t) v\right\rangle \geq \gamma_{j} e^{-2 \Gamma t}\|v\|^{2}$ holds for every $v \in \mathbb{R}^{n}$. We conclude from Lemma 5.13 for all $s, t \in[0, T]$

$$
\begin{aligned}
& \left\|X_{j}(s)^{\frac{1}{2}}-X_{j}(t)^{\frac{1}{2}}\right\|_{\mathrm{op}} \leq \operatorname{const}\left(\gamma_{j}\right) \cdot\left\|X_{j}(s)-X_{j}(t)\right\|_{\mathrm{op}} \\
& \quad \leq \operatorname{const}\left(\Gamma, Q_{u}, X_{0 j}, T\right) \cdot|s-t| .
\end{aligned}
$$

Finally, Lemma 5.8 (3.) and the LIPSCHITZ continuity of $x_{j}(\cdot)$ guarantee that $E_{j}$ : $[0, T] \rightsquigarrow \mathbb{R}^{n}$ is LIPSCHITZ continuous (w.r.t. $\mathbb{d}$ ).

(2.) Due to Lemma 5.11, we conclude from Lemma 5.7 (1.) that $\mathcal{E}_{\cap}:[0, T] \rightsquigarrow \mathbb{R}^{n}$ is also LIPSCHITZ continuous. Hence, Assumptions 3.2 (iii) and 3.3 (ii') guarantee the continuity of $\mathcal{A}\left(\cdot, \mathcal{E}_{\cap}(\cdot)\right)$ and $\mathcal{B}\left(\cdot, \mathcal{E}_{\cap}(\cdot)\right):[0, T] \longrightarrow \mathbb{R}^{n \times n}$. Finally, Proposition 3.1 (2.) (a) states the claimed limit for each $t \in[0, T)$.

\subsection{Approximating the Set Evolution Solution with Arbitrary Precision (Proposition 3.6)}

The proof of [12, Theorem] provides several useful supplementary details.

Lemma 5.17 ([12]) Let $M \subset \mathbb{R}^{n}$ be a bounded convex subset with nonempty interior and boundary of class $C^{1}$. Fix $\varepsilon \in\left(0, \frac{1}{32}\right)$ arbitrarily. Then, the following statements hold:

(1.) There exist finitely many $x_{1}, \ldots, x_{N} \in \partial M$ and unit vectors $v_{1}, \ldots, v_{N} \in$ $\mathbb{R}^{n}$ such that for every $j \in\{1, \ldots, N\}, v_{j}$ is normal to $M$ in $x_{j}$ and $\operatorname{co}\left\{x_{1}, \ldots, x_{N}\right\} \subset \bar{M} \subset \operatorname{co}\left\{x_{1}+\varepsilon v_{1}, \ldots, x_{N}+\varepsilon v_{N}\right\}$. In particular, $\mathbb{d}\left(\bar{M}\right.$, co $\left.\left\{x_{1}+\varepsilon v_{1}, \ldots, x_{N}+\varepsilon v_{N}\right\}\right) \leq \varepsilon$.

(2.) Choose $x_{1}, \ldots, x_{N} \in \partial M$ and normal unit vectors $v_{1}, \ldots, v_{N} \in \mathbb{R}^{n}$ such that for each $j \in\{1, \ldots, N\}$,

$$
\min _{k \neq j}\left\|v_{j}-v_{k}\right\|\left(1+\frac{3}{16} \frac{\left\|v_{j}-v_{k}\right\|^{2}}{1-\left\|v_{j}-v_{k}\right\|^{2}}\right)<\sqrt{2 \varepsilon}
$$

Then, $M$ is contained in the convex hull of $\left\{x_{j}+\varepsilon v_{j} \mid j \in\{1, \ldots, N\}\right\}$.

The following lemma results from the observation that every compact convex set in $\mathbb{R}^{n}$ is of arbitrary positive reach (in the sense of FEDERER) (e.g., [21, Ch. 6, $\S 6 \&$ Theorem 8.1 and Ch. 7, Theorem 7.1]).

Lemma 5.18 Let $M \subset \mathbb{R}^{n}$ be nonempty, compact and convex. For each $\varepsilon>0$, the boundary of $\overline{\mathbb{B}}_{\varepsilon}(M) \stackrel{\text { Def. }}{=}\left\{x \in \mathbb{R}^{n} \mid \operatorname{dist}(x, M) \leq \varepsilon\right\}$ is of class $C^{1,1}$.

Proof of Proposition 3.6 Fix any $\varepsilon \in\left(0, \frac{1}{32}\right)$ and set $\widetilde{r}:=\max \left\{1,\left(\mathbb{d}\left(\{0\}, K_{0}\right)+e^{\Gamma T}\right)\right.$. $\left.e^{\Gamma T}, \mathbb{d}(\{0\}, U)\right\} \geq 1, \widehat{\varepsilon}:=\frac{\varepsilon}{2} \cdot e^{-4 \widetilde{r} \Lambda T \cdot \exp ((2 \widetilde{r} \Lambda+\Gamma) T)} \in\left(0, \frac{\varepsilon}{2}\right), \widetilde{\varepsilon}:=\frac{3}{4} e^{-\Gamma T \cdot \widehat{\varepsilon} \in}$ $\left(0, \frac{3}{8} \varepsilon\right)$.

Step 1 Choosing $N \in \mathbb{N}$ and the initial unit vectors $\ell_{0 j} \in \mathbb{R}^{n}(j=1, \ldots, N)$. 
Due to Lemma $5.18, \overline{\mathbb{B}} \widetilde{\varepsilon} / 4\left(K_{0}\right) \subset \mathbb{R}^{n}$ has the boundary of class $C^{1,1}$ and so, it satisfies the assumptions of Lemma 5.17 in particular. Hence, there exist finitely many points $\zeta_{1}, \ldots, \zeta_{N} \in \partial \overline{\mathbb{B}}_{\widetilde{\varepsilon} / 4}\left(K_{0}\right)$ and unit vectors $\ell_{01}, \ldots, \ell_{0 N} \in \mathbb{R}^{n}$ such that

- for every $j \in\{1, \ldots, N\}, \ell_{0 j}$ is normal to $\overline{\mathbb{B}} \widetilde{\varepsilon} / 4\left(K_{0}\right)$ in $\zeta_{j}$,

- $\operatorname{co}\left\{\zeta_{1}, \ldots, \zeta_{N}\right\} \subset \bar{B}_{\widetilde{\varepsilon} / 4}\left(K_{0}\right) \subset \operatorname{co}\left\{\zeta_{1}+\frac{\widetilde{\varepsilon}}{4} \ell_{01}, \ldots, \zeta_{N}+\frac{\widetilde{\varepsilon}}{4} \ell_{0 N}\right\}$,

- for every $j \in\{1, \ldots, N\}$, $\operatorname{dist}\left(\ell_{0 j},\left\{\ell_{0 k} \mid k \neq j\right\}\right)<\widetilde{\varepsilon}$ and $\operatorname{dist}\left(\zeta_{j},\left\{\zeta_{k} \mid k \neq\right.\right.$ $j\})<\frac{\widetilde{\varepsilon}}{4}$.

For each $j \in\{1, \ldots, N\}, \xi_{0 j}:=\zeta_{j}-\frac{\widetilde{\varepsilon}}{4} \ell_{0 j}$ belongs to $\partial K_{0}$ and, $\ell_{0 j}$ is normal to $K_{0}$ in $\xi_{0 j}$ as a consequence of [21, Ch. 6, Theorem 6.2 (iii)]. Moreover, the triangle inequality leads to $\operatorname{dist}\left(\xi_{0 j}, \quad\left\{\xi_{0 k} \mid k \neq j\right\}\right)<\widetilde{\varepsilon}$.

Step 2 Constructing the ellipsoid-valued $E_{j}(\cdot)$ and the auxiliary tube $R(\cdot)$.

$K:[0, T] \rightsquigarrow \mathbb{R}^{n}$ denotes the solution to $\operatorname{IVP}(6)$.

Proposition 3.3 provides unique solutions $\ell_{j}, x_{j}:[0, T] \longrightarrow \mathbb{R}^{n}, X_{j}:[0, T] \longrightarrow$ $\mathbb{R}^{n \times n}(j=1, \ldots, N)$ to ODE system (7) with initial values $\ell_{j}(0)=\ell_{0 j}, x_{j}(0)=x_{0 j}$, $X_{j}(0)=X_{0 j}$ (and abbreviations (8)). According to Corollary 3.4, the intersection map $\mathcal{E}_{\cap}:=\bigcap_{j=1}^{N} \mathcal{E}\left(x_{j}, X_{j}\right):[0, T] \rightsquigarrow \mathbb{R}^{n}$ satisfies $K(t) \subset \mathcal{E}_{\cap}(t)$ for every $t \in[0, T]$.

Define $A:=\mathcal{A}\left(\cdot, \mathcal{E}_{\cap}\right), B:=\mathcal{B}\left(\cdot, \mathcal{E}_{\cap}\right):[0, T] \longrightarrow \mathbb{R}^{n \times n}$ and consider $x^{\prime} \in A(\cdot) x+$ $B(\cdot) U$ in $[0, T]$. Let $R(t) \subset \mathbb{R}^{n}$ denote its reachable set of $K_{0}$ at each time $t \in[0, T]$. Proposition 3.1 (2.) guarantees for every $j \in\{1, \ldots, N\}$ and $t \in[0, T]$

- $R(t) \subset E_{j}(t)$.

- $E_{j}(t)$ is minimal in the class of ellipsoids w.r.t. set inclusions, i.e., there is no ellipsoid $\widetilde{E} \subset \mathbb{R}^{n}$ with $R(t) \subset \widetilde{E} \varsubsetneqq E_{j}(t)$.

- $\ell_{j}(t)$ is normal to $R(t)$ and $E_{j}(t)$ in $\xi_{j}(t):=x_{j}(t)+\left\langle\ell_{j}(t), X_{j}(t) \ell_{j}(t)\right\rangle^{-\frac{1}{2}} X_{j}(t)$ $\ell_{j}(t) \in \partial R(t) \cap \partial E_{j}(t)$.

Step 3 An upper bound of the POMPEIU-HAUSDORFF distance between $R(t)$ and $\mathcal{E}_{\cap}(t)$.

For each $j \in\{1, \ldots, N\}$, the choice of $\ell_{j}(0)=\ell_{0 j}$ in STEP 1 implies $\xi_{j}(0)=$ $\xi_{0 j} \in \partial K_{0} \stackrel{\text { Def. }}{=} \partial \mathcal{E}\left(x_{0}, X_{0}\right)$. Moreover, there exists $k \in\{1, \ldots, N\} \backslash\{j\}$ satisfying $\left\|\ell_{j}(t)-\ell_{k}(t)\right\|<\frac{3}{4} \widehat{\varepsilon}$ for all $t \in[0, T]$. Indeed, STEP 1 provides an index $k \neq j$ with $\left\|\ell_{0 j}-\ell_{0 k}\right\|<\widetilde{\varepsilon}$. Hence, we conclude from ODE system (7) and Assumption 3.3 (iv') for each $t \in[0, T]$

$$
\begin{aligned}
\left\|\ell_{j}(t)-\ell_{k}(t)\right\| & \leq \int_{0}^{t}\left\|-\mathcal{A}\left(s, \mathcal{E}_{\cap}(s)\right)^{\top} \ell_{j}(s)+\mathcal{A}\left(s, \mathcal{E}_{\cap}(s)\right)^{\top} \ell_{k}(s)\right\| \mathrm{d} s \\
& \leq \int_{0}^{t} \Gamma\left\|\ell_{j}(s)-\ell_{k}(s)\right\| \mathrm{d} s .
\end{aligned}
$$

GRONWALL's inequality leads to $\left\|\ell_{j}(t)-\ell_{k}(t)\right\| \leq\left\|\ell_{0 j}-\ell_{0 k}\right\| e^{\Gamma T}<\frac{3}{4} \widehat{\varepsilon}$. In particular, we have

$$
\left\|\ell_{j}(t)-\ell_{k}(t)\right\|\left(1+\frac{3}{16} \frac{\left\|\ell_{j}(t)-\ell_{k}(t)\right\|^{2}}{1-\left\|\ell_{j}(t)-\ell_{k}(t)\right\|^{2}}\right) \leq \frac{4}{3}\left\|\ell_{j}(t)-\ell_{k}(t)\right\|<\widehat{\varepsilon}<\sqrt{2 \frac{3 \widehat{\varepsilon}}{4}} .
$$


For each $t \in[0, T]$, the boundary of $\overline{\mathbb{B}} \widehat{\varepsilon} / 4(R(t))$ is of class $C^{1,1}$ due to Lemma 5.18 and so, we can apply Lemma $5.17\left(2\right.$.) to $\xi_{j}(t)+\frac{\widehat{\varepsilon}}{4} \ell_{j}(t) \in \partial \overline{\mathbb{B}} \widehat{\varepsilon} / 4(R(t))$, the normals $\ell_{j}(t)(j=1, \ldots, N)$ and $\frac{3}{4} \widehat{\varepsilon} \in\left(0, \frac{1}{32}\right)$ :

$\bigcup_{j=1}^{N}\left\{\xi_{j}(t)\right\} \subset R(t) \subset \overline{\mathbb{B}} \widehat{\varepsilon} / 4(R(t)) \subset \operatorname{co} \bigcup_{j=1}^{N}\left\{\xi_{j}(t)+\widehat{\varepsilon} \ell_{j}(t)\right\}$.

These arguments (with the same points and normals) also hold for $\mathcal{E}_{\cap}(t)$, i.e.,

$$
\bigcup_{j=1}^{N}\left\{\xi_{j}(t)\right\} \subset \mathcal{E}_{\cap}(t) \subset \overline{\mathbb{B}} \widehat{\varepsilon} / 4\left(\mathcal{E}_{\cap}(t)\right) \subset \operatorname{co} \bigcup_{j=1}^{N}\left\{\xi_{j}(t)+\widehat{\varepsilon} \ell_{j}(t)\right\} .
$$

Hence, we obtain $\mathbb{d}\left(R(t), \mathcal{E}_{\cap}(t)\right) \leq \widehat{\varepsilon}$ for every $t \in[0, T]$.

Step 4 An upper bound of the POMPEIU-HAUSDORFF distance between $K(t)$ and $R(t)$.

The tube $R:[0, T] \rightsquigarrow \mathbb{R}^{n}$ of reachable sets is a solution of $\stackrel{\circ}{R}(t)=\mathcal{A}\left(t, \mathcal{E}_{\cap}(t)\right) x+$ $\mathcal{B}\left(t, \mathcal{E}_{\cap}(t)\right) U$ with $R(0)=K_{0}$ (in the sense of Definition 2.3). Lemma 5.2 (1.) guarantees $K(t) \cup R(t) \subset \overline{\mathbb{B}}_{\widetilde{r}}$ for all $t \in[0, T]$. Hence, we conclude from Proposition 2.5 and the Assumptions 3.2 (iii), 3.3 (iv') for every $t \in[0, T]$

$$
\begin{aligned}
\mathbb{d}(K(t), R(t)) \leq & \int_{0}^{t} \sup _{\substack{x \in \overline{\mathbb{B}}_{\tilde{r}} \\
u \in U}} \| \mathcal{A}(s, \quad K(s)) x+\mathcal{B}(s, \quad K(s)) u \\
& -\mathcal{A}\left(s, \mathcal{E}_{\cap}(s)\right) x-\mathcal{B}\left(s, \mathcal{E}_{\cap}(s)\right) u \| e^{(2 \widetilde{r} \Lambda+\Gamma)(t-s)} d s \\
\leq & 2 \widetilde{r} e^{(2 \widetilde{r} \Lambda+\Gamma) T} \Lambda \cdot \int_{0}^{t} \mathbb{d}\left(K(s), \mathcal{E}_{\cap}(s)\right) \mathrm{d} s .
\end{aligned}
$$

STEP 3 leads to $\mathbb{d}\left(K(t), \mathcal{E}_{\cap}(t)\right) \leq 2 \widetilde{r} e^{(2 \tilde{r} \Lambda+\Gamma) T} \Lambda \cdot \int_{0}^{t} \mathbb{d}\left(K(s), \mathcal{E}_{\cap}(s)\right) \mathrm{d} s+\widehat{\varepsilon}$. Finally, GRONWALL's inequality states for every $t \in[0, T]$ (due to $\tilde{r} \geq 1$ )

$$
\begin{aligned}
& \mathbb{d}\left(K(t), \mathcal{E}_{\cap}(t)\right) \\
& \quad \leq \widehat{\varepsilon} \cdot\left(1+2 \widetilde{r} e^{(2 \tilde{r} \Lambda+\Gamma) T} \Lambda \cdot \int_{0}^{t} \exp \left(2 \widetilde{r} e^{(2 \tilde{r} \Lambda+\Gamma) T} \Lambda(t-s)\right) \mathrm{d} s\right) \\
& \leq \widehat{\varepsilon} \cdot e^{4 \widetilde{r} \Lambda T \cdot e^{(2 \tilde{r} \Lambda+\Gamma) T}}<\varepsilon .
\end{aligned}
$$

\section{Conclusions}

Ellipsoids represent a well-established approach of approximation in control theory and related optimization problems (like the OFC by KURZHANSKI and VARAIYA in [35], [36, Ch. 10]). Now they are used for external approximations of convex-valued solutions to so-called set evolution equations. In a word, we focus on reachable sets to linear time-variant control systems whose coefficient matrices depend on their own reachable set. (This very general form of feedback can be formulated equivalently 
in terms of integral funnel equations, PANASYUK's quasidifferential equations and AUBIN's morphological equations, see Propositions 2.1 and 2.7). In regard to future applications, the main result consists in sufficient conditions on the coefficients such that the convex solution values can be approximated as intersections of finitely many ellipsoids with arbitrary precision. Their respective centers and matrices are characterized by a nonlinear ODE system (Sect. 3) and so, they can be calculated rather quickly.

Acknowledgments TL was partially supported by the Air Force Office of Scientific Research under Award Number FA9550-18-1-0254.

Funding Open Access funding enabled and organized by Projekt DEAL.

Open Access This article is licensed under a Creative Commons Attribution 4.0 International License, which permits use, sharing, adaptation, distribution and reproduction in any medium or format, as long as you give appropriate credit to the original author(s) and the source, provide a link to the Creative Commons licence, and indicate if changes were made. The images or other third party material in this article are included in the article's Creative Commons licence, unless indicated otherwise in a credit line to the material. If material is not included in the article's Creative Commons licence and your intended use is not permitted by statutory regulation or exceeds the permitted use, you will need to obtain permission directly from the copyright holder. To view a copy of this licence, visit http://creativecommons.org/licenses/by/4.0/.

\section{References}

1. Aubin, J.P.: Viability Theory. Systems \& Control: Foundations \& Applications. Birkhäuser, Boston, MA (1991)

2. Aubin, J.P.: A note on differential calculus in metric spaces and its applications to the evolution of tubes. Bull. Pol. Acad. Sci. Math. 40(2), 151-162 (1992)

3. Aubin, J.P.: Mutational equations in metric spaces. Set-Valued Anal. 1(1), 3-46 (1993). https://doi. org/10.1007/BF01039289

4. Aubin, J.P.: Mutational and Morphological Analysis. Systems \& Control: Foundations \& Applications. Tools for Shape Evolution and Morphogenesis. Birkhäuser, Boston, MA (1999). https://doi.org/10. 1007/978-1-4612-1576-9 (Tools for shape evolution and morphogenesis)

5. Aubin, J.P., Frankowska, H.: Set-Valued Analysis, Systems \& Control: Foundations \& Applications, vol. 2. Birkhäuser, Boston, MA (1990)

6. Baier, R., Gerdts, M., Xausa, I.: Approximation of reachable sets using optimal control algorithms. Numer. Algebra Control Optim. 3(3), 519-548 (2013). https://doi.org/10.3934/naco.2013.3.519

7. Beer, G.: Topologies on Closed and Closed Convex Sets, Mathematics and Its Applications, vol. 268. Kluwer Academic Publishers, Dordrecht (1993). https://doi.org/10.1007/978-94-015-8149-3

8. Beyn, W.J., Rieger, J.: Numerical fixed grid methods for differential inclusions. Computing 81(1), 91-106 (2007). https://doi.org/10.1007/s00607-007-0240-4

9. Bhatia, R.: Some inequalities for norm ideals. Commun. Math. Phys. 111(1), 33-39 (1987)

10. Blanchini, F., Miani, S.: Set-Theoretic Methods in Control, 2nd edn. Systems \& Control: Foundations \& Applications. Birkhäuser/Springer, Cham (2015). https://doi.org/10.1007/978-3-319-17933-9

11. Bogachev, V.I.: Measure Theory, vols. I, II. Springer, Berlin (2007). https://doi.org/10.1007/978-3540-34514-5

12. Bronšter̆n, E.M., Ivanov, L.D.: The approximation of convex sets by polyhedra. Sib. Math. J. 16(5), 852-853 (1975). https://doi.org/10.1007/BF00967115

13. Chernous'ko, F.L.: Ellipsoidal bounds for sets of attainability and uncertainty in control problems. Optimal Control Appl. Methods 3(2), 187-202 (1982). https://doi.org/10.1002/oca.4660030207

14. Chernous'ko, F.L.: State Estimation for Dynamic Systems. CRC Press, Boca Raton, FL (1994)

15. Chernousko, F.L.: Optimal ellipsoidal estimates of uncertain systems: an overview and new results. In: Coping With Uncertainty, Lecture Notes in Econometrics and Mathematical Systems, vol. 633, pp. 141-161. Springer, Berlin (2010). https://doi.org/10.1007/978-3-642-03735-1_7 
16. Chernousko, F.L., Rokityanskii, D.Y.: Ellipsoidal bounds on reachable sets of dynamical systems with matrices subjected to uncertain perturbations. J. Optim. Theory Appl. 104(1), 1-19 (2000). https://doi. org/10.1023/A:1004687620019

17. Colombo, R.M., Lorenz, T., Pogodaev, N.I.: On the modeling of moving populations through set evolution equations. Discrete Contin. Dyn. Syst. Ser. A 35(1), 73-98 (2015). https://doi.org/10.3934/ dcds.2015.35.73

18. Colombo, R.M., Pogodaev, N.: Confinement strategies in a model for the interaction between individuals and a continuum. SIAM J. Appl. Dyn. Syst. 11(2), 741-770 (2012). https://doi.org/10.1137/ 110854321

19. Colombo, R.M., Pogodaev, N.: On the control of moving sets: positive and negative confinement results. SIAM J. Control. Optim. 51(1), 380-401 (2013). https://doi.org/10.1137/12087791X

20. Delfour, M.C., Zolésio, J.P.: Velocity method and Lagrangian formulation for the computation of the shape Hessian. SIAM J. Control. Optim. 29(6), 1414-1442 (1991). https://doi.org/10.1137/0329072

21. Delfour, M.C., Zolésio, J.P.: Shapes and Geometries, Advances in Design and Control, vol. 22, 2nd edn. Society for Industrial and Applied Mathematics (SIAM), Philadelphia, PA (2011). https://doi.org/ $10.1137 / 1.9780898719826$

22. Doyen, L.: Mutational equations for shapes and vision-based control. J. Math. Imaging Vis. 5(2), 99-109 (1995). https://doi.org/10.1007/BF01250522

23. Filippova, T.F., Kurzhanski, A.B., Sugimoto, K., Vályi, I.: Ellipsoidal calculus, singular perturbations and the state estimation problems for uncertain systems. J. Math. Syst. Estim. Control 6(3), 323-338 (1996)

24. Fremlin, D.H.: Measurable functions and almost continuous functions. Manuscr. Math. 33(3-4), 387405 (1980/81). https://doi.org/10.1007/BF01798235

25. Goffin, J.L., Hoffman, A.J.: On the relationship between the Hausdorff distance and matrix distances of ellipsoids. Linear Algebra Appl. 52(53), 301-313 (1983). https://doi.org/10.1016/00243795(83)80021-4

26. Gorre, A.: Évolutions de tubes opérables gouvernées par des équations mutationnelles. Ph.D. thesis. Université Paris IX Dauphine (1996). http://www.theses.fr/1996PA090076 (Thèse de doctorat dirigée par Aubin, Jean-Pierre, CEREMADE)

27. Gorre, A.: Evolutions of tubes under operability constraints. J. Math. Anal. Appl. 216(1), 1-22 (1997). https://doi.org/10.1006/jmaa.1997.5476

28. Grammel, G.: Towards fully discretized differential inclusions. Set-Valued Anal. 11(1), 1-8 (2003). https://doi.org/10.1023/A:1021981217050

29. Higham, N.J.: Functions of Matrices. Society for Industrial and Applied Mathematics (SIAM), Philadelphia, PA (2008). https://doi.org/10.1137/1.9780898717778

30. Hu, S., Papageorgiou, N.S.: Handbook of Multivalued Analysis, vol. I, Mathematics and Its Applications, vol. 419. Kluwer Academic Publishers, Dordrecht (1997) (Theory)

31. Komarov, V.A.: Estimates of sets of attainability for linear systems. Izv. Akad. Nauk SSSR Ser. Mat. 48(4), 865-879 (1984)

32. Kupka, J., Prikry, K.: The measurability of uncountable unions. Am. Math. Mon. 91(2), 85-97 (1984). https://doi.org/10.2307/2322101

33. Kurzhanski, A., Vályi, I.: Ellipsoidal Calculus for Estimation and Control. Systems \& Control: Foundations \& Applications. Birkhäuser, Boston, MA (1997). https://doi.org/10.1007/978-1-46120277-6

34. Kurzhanski, A.B., Filippova, T.F.: On the theory of trajectory tubes-a mathematical formalism for uncertain dynamics, viability and control. In: Kurzhanski, A.B. (ed.) Advances in Nonlinear Dynamics and Control: A Report From Russia, Progress in Systems Control Theory, vol. 17, pp. 122-188. Birkhäuser, Boston, MA (1993)

35. Kurzhanski, A.B., Varaiya, P.: Optimization of output feedback control under set-membership uncertainty. J. Optim. Theory Appl. 151(1), 11-32 (2011). https://doi.org/10.1007/s10957-011-9861-z

36. Kurzhanski, A.B., Varaiya, P.: Dynamics and Control of Trajectory Tubes. Systems \& Control: Foundations \& Applications. Birkhäuser/Springer, Cham (2014). https://doi.org/10.1007/978-3-31910277-1

37. Lakshmikantham, V., Gnana Bhaskar, T., Vasundhara Devi, J.: Theory of Set Differential Equations in Metric Spaces. Cambridge Scientific Publishers, Cambridge (2006) 
38. Lee, D., Keimer, A., Bayen, A.M., Tomlin, C.J.: Hamilton-Jacobi formulation for state-constrained optimal control and zero-sum game problems. In: 2020 59th IEEE Conference on Decision and Control (CDC), pp. 1078-1085 (2020). https://doi.org/10.1109/CDC42340.2020.9304334

39. Lorenz, T.: Set-valued maps for image segmentation. Comput. Vis. Sci. 4(1), 41-57 (2001). https:// doi.org/10.1007/s007910100056

40. Lorenz, T.: Morphological control problems with state constraints. SIAM J. Control. Optim. 48(8), 5510-5546 (2010). https://doi.org/10.1137/090752183

41. Lorenz, T.: Mutational Analysis. Lecture Notes in Mathematics, vol. 1996. Springer, Berlin (2010)

42. Lorenz, T.: Differential equations for closed sets in a Banach space: survey and extension. Vietnam J. Math. 45(1-2), 5-49 (2017). https://doi.org/10.1007/s10013-016-0195-x

43. Lorenz, T.: A viability theorem for set-valued states in a Hilbert space. J. Math. Anal. Appl. 457(2), 1502-1567 (2018). https://doi.org/10.1016/j.jmaa.2017.08.011

44. Martynyuk, A.A.: Qualitative Analysis of Set-Valued Differential Equations. Birkhäuser/Springer, Cham (2019). https://doi.org/10.1007/978-3-030-07644-3

45. Mitchell, I.M.: In: ProQuest, L.L.C., Ann Arbor, M.I. (eds.) Application of Level Set Methods to Control and Reachability Problems in Continuous and Hybrid Systems. Thesis (Ph.D.)-Stanford University (2002)

46. Mitchell, I.M., Bayen, A.M., Tomlin, C.J.: A time-dependent Hamilton-Jacobi formulation of reachable sets for continuous dynamic games. IEEE Trans. Autom. Control 50(7), 947-957 (2005). https:// doi.org/10.1109/TAC.2005.851439

47. Moreau, J.J.: Intersection of moving convex sets in a normed space. Math. Scand. 36, 159-173 (1975). https://doi.org/10.7146/math.scand.a-11569

48. Panasjuk, A.I., Panasjuk, V.I.: An equation generated by a differential inclusion. Math. Notes 27, 213-218 (1980). https://doi.org/10.1007/BF01140170

49. Panasyuk, A.I.: Quasidifferential equations in a metric space. Differ. Uravn. 21(8), 1344-1353 (1985)

50. Panasyuk, A.I.: Equations of the dynamics of sets of reachability in problems of optimization and control under conditions of uncertainty. Prikl. Mat. Mekh. 50(4), 531-543 (1986). https://doi.org/10. 1016/0021-8928(86)90001-8

51. Panasyuk, A.I.: Qualitative dynamics of sets that are defined by differential inclusions. Mat. Zametki 45(1), 80-88 (1989). https://doi.org/10.1007/BF01158717

52. Panasyuk, A.I.: Equations of attainable set dynamics. I. Integral funnel equations. J. Optim. Theory Appl. 64(2), 349-366 (1990). https://doi.org/10.1007/BF00939453

53. Panasyuk, A.I.: Properties of solutions of a quasidifferential approximation equation and an equation of an integral funnel. Differ. Uravn. 28(9), 1537-1544, 1652-1653 (1992)

54. Panasyuk, A.I.: Quasidifferential equations in a complete metric space under Carathéodory-type conditions. I. Differ. Uravn. 31(6), 962-972 (1995)

55. Quincampoix, M., Veliov, V.M.: Solution tubes to differential inclusions within a collection of sets. Control Cybernet. 31(3), 847-862 (2002)

56. Ricceri, B., Villani, A.: Separability and Scorza-Dragoni’s property. Matematiche (Catania) 37(1), 156-161 (1985/1982)

57. Riedl, W., Baier, R., Gerdts, M.: Optimization-based subdivision algorithm for reachable sets. J. Comput. Dyn. 8(1), 99-130 (2021). https://doi.org/10.3934/jcd.2021005

58. Searcóid, M.Ó.: Metric Spaces. Springer Undergraduate Mathematics Series, Springer, London (2007). https://doi.org/10.1007/978-1-84628-627-8

59. Sokołowski, J., Zolésio, J.P.: Introduction to Shape Optimization. Springer Series in Computational Mathematics, vol. 16. Springer, Berlin (1992). https://doi.org/10.1007/978-3-642-58106-9

60. Stein, E.M., Shakarchi, R.: Real Analysis, Princeton Lectures in Analysis, vol. 3. Princeton University Press, Princeton, NJ (2005)

61. Tolstonogov, A.A.: Equation of the solution funnel of a differential inclusion. Math. Notes 32(6), 908-914 (1983). https://doi.org/10.1007/BF01145876

62. Tolstonogov, A.A.: Differential Inclusions in a Banach Space, Mathematics and Its Applications, vol. 524. Kluwer Academic Publishers, Dordrecht (2000). https://doi.org/10.1007/978-94-015-94905 (Translated from the 1986 Russian original and revised by the author)

63. Varaiya, P., Kurzhanski, A.B.: Ellipsoidal methods for dynamics and control. I. J. Math. Sci. 139(5), 6863-6901 (2006) 
64. Walter, W.: Ordinary Differential Equations, Graduate Texts in Mathematics, vol. 182. Springer, New York (1998). https://doi.org/10.1007/978-1-4612-0601-9 (Translated from the sixth German (1996) edition by Russell Thompson, Readings in Mathematics)

65. Xausa, I., Baier, R., Bokanowski, O., Gerdts, M.: Computation of avoidance regions for driver assistance systems by using a Hamilton-Jacobi approach. Optimal Control Appl. Methods 41(2), 668-689 (2020). https://doi.org/10.1002/oca.2565

66. Zhao, J., Song, B., Xi, N., Sun, L., Chen, H., Jia, Y.: Non-vector space approach for nanoscale motion control. Autom. J. IFAC 50(7), 1835-1842 (2014). https://doi.org/10.1016/j.automatica.2014.04.018

Publisher's Note Springer Nature remains neutral with regard to jurisdictional claims in published maps and institutional affiliations. 